\title{
EFFECT OF A SYMMETRIC CONTRACTION ON THE CONCENTRATION PROFILES OF A PARTICLE- \\ LADEN SLURRY
}

\author{
By \\ ABHISHEK DESHPANDE \\ Bachelor of Science in Mechanical Engineering \\ Jawaharlal Nehru Technological University \\ Hyderabad, Andhra Pradesh
}

2008
Submitted to the Faculty of the
Graduate College of the
Oklahoma State University
in partial fulfillment of the requirements for the Degree of MASTER OF SCIENCE
December, 2010




\section{EFFECT OF A SYMMETRIC CONTRACTION ON THE CONCENTRATION PROFILES OF A PARTICLE-}

\section{LADEN SLURRY}

Thesis Approved:

Dr. Frank W. Chambers

Thesis Adviser

Dr. Afshin J. Ghajar

Dr. Jamey D. Jacob

Dr. Mark E. Payton

Dean of the Graduate College 


\section{ACKNOWLEDGEMENTS}

I would like to thank my adviser, Dr. Frank W. Chambers, for making this seemingly turbulent voyage as laminar as possible. By answering my most rudimentary of queries with an unwavering temperament, Dr. Chambers helped me overcome numerous dilemmas that spanned a great spectrum. For this I owe him my eternal gratitude.

I would also like to thank Dr. Afshin J. Ghajar and Dr. Jamey D. Jacob for considering this work worthy enough to be associated with. I am thoroughly grateful to them as they lent their much needed expertise to push this work closer to perfection.

Unflinching support from my peer group always provided me with the incentive to go the extra mile. I would like to thank Karthik Ramisetty for his priceless suggestions. I am grateful to Rama Krishna Chatanya, Gaurav Sharma, and Vamsee Pasangulapati for their insightful advice when it came to the computational part of the endeavor. I must thank Ms. Diane Compton, Ashish Singh, Anugu Arjun Reddy, Vineeth Yadav, and Vamsi Karthik Kommineni for their valuable suggestions relative to the formatting and editing details.

I must especially thank my parents (Dr. Ashok Kumar Deshpande and Mrs. Sushma Deshpande) and my sister (Avani Deshpande) for breathing life into me whenever the chips were down. Despite being thousands of miles away, their support resonates in every keystroke, mouse click, and page flip that went into this work.

Finally, I would like to thank the force that everyone looks up to. The presence of this paragraph is a testimony of the fact that the force was/is on my side. Last but not the least (apologize for not trying harder to avoid the cliché); I would like to thank all the people who

directly or indirectly devoted their time to this endeavor. They are the unsung heroes, and they know who they are. 


\section{TABLE OF CONTENTS}

Chapter

Page

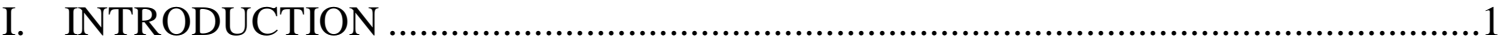

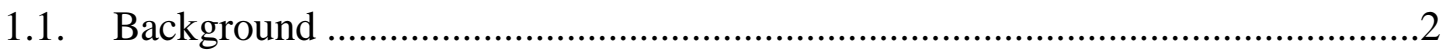

1.2. Problem statement and presentation ...........................................................

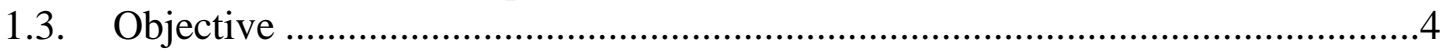

II. REVIEW OF LITERATURE .....................................................................

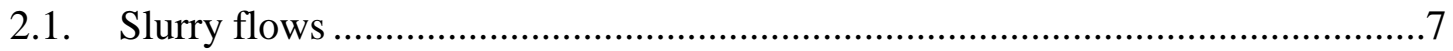

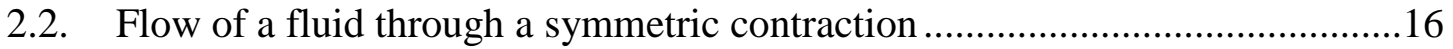

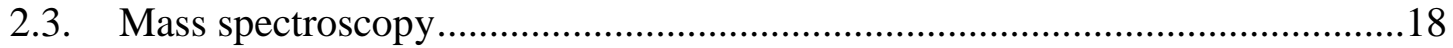

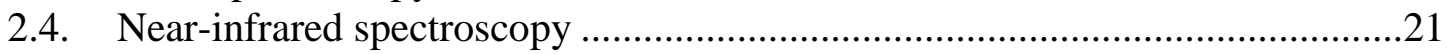

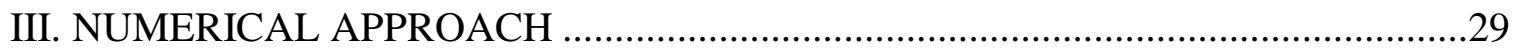

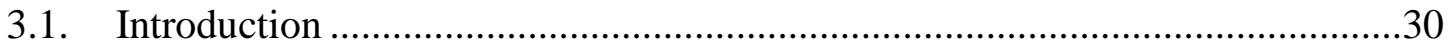

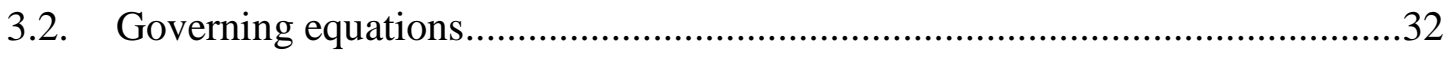

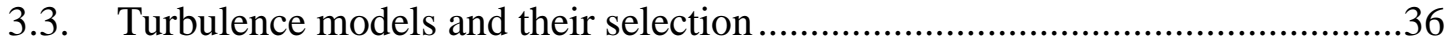

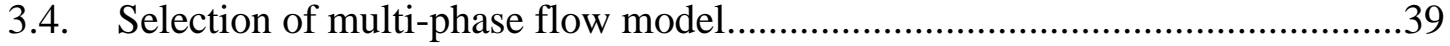

3.4.1. Modeling with the mixture model...............................................42

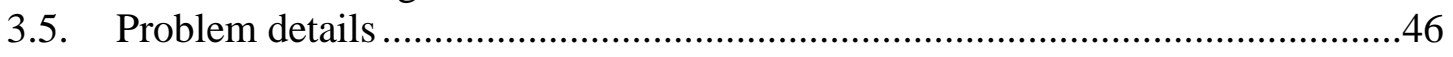

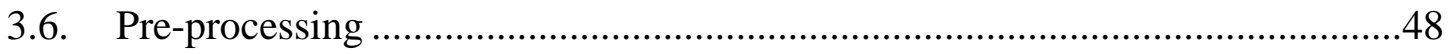

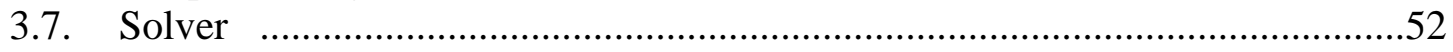

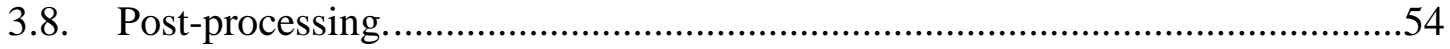




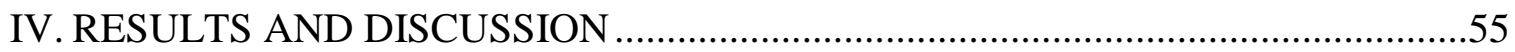

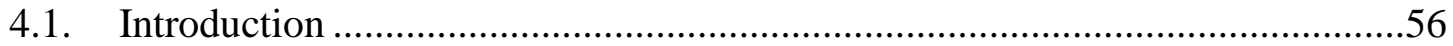

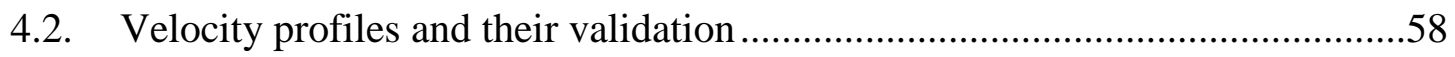

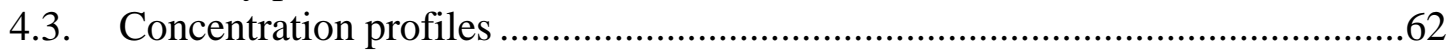

4.3.1. Validation of the concentration profiles .......................................63

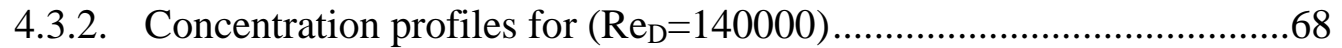

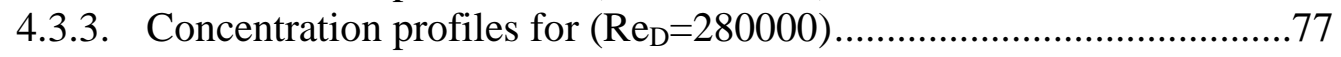

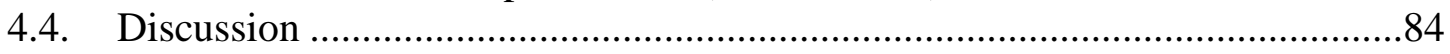

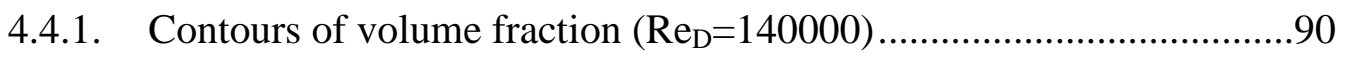

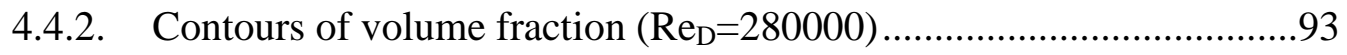

V. CONCLUSION AND RECOMMENDATIONS …........................................... 99

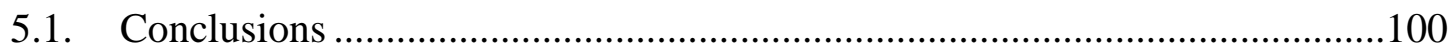

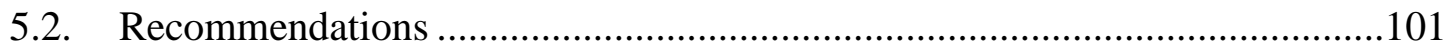

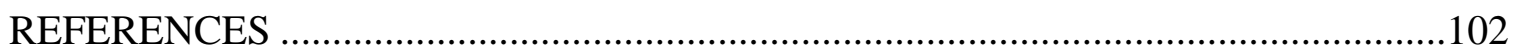

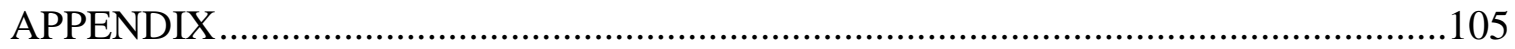




\section{NOMENCLATURE}

\begin{tabular}{|c|c|}
\hline C: & Initial Efflux concentration by volume \\
\hline D: & Diameter of pipe, (m) \\
\hline$d_{p}:$ & Particle size, $(\mu \mathrm{m})$ \\
\hline $\mathrm{El}_{\text {turbulent }}:$ & Entrance length for fully developed flow \\
\hline f: & Friction factor \\
\hline k: & Turbulent kinetic energy, $\left(\mathrm{m}^{2} / \mathrm{s}^{2}\right)$ \\
\hline $\mathrm{L}_{\mathrm{s}}:$ & Characteristic length scale, (m) \\
\hline 1: & Length of pipe, $(\mathrm{m})$ \\
\hline$\Delta \mathrm{p}:$ & Pressure drop, (Kpa) \\
\hline p': & Pressure fluctuation, (Kpa) \\
\hline $\mathrm{Pe}_{\gamma:}$ & Peclet number of a slurry \\
\hline $\operatorname{Re}_{\gamma}:$ & Reynolds number for a slurry \\
\hline $\operatorname{Re}_{\mathrm{D}}$ : & Pipe Reynolds number \\
\hline
\end{tabular}

St: $\quad$ Stokes number 


\begin{tabular}{|c|c|}
\hline $\mathrm{T}^{\prime}:$ & Temperature fluctuation, $(\mathrm{K})$ \\
\hline $\mathrm{U}_{\mathrm{cl}}:$ & Center line velocity, $(\mathrm{m} / \mathrm{s})$ \\
\hline u': & Velocity fluctuation in X-direction, $(\mathrm{m} / \mathrm{s})$ \\
\hline V: & Mean velocity of fluid, $(\mathrm{m} / \mathrm{s})$ \\
\hline $\mathrm{V}_{\mathrm{d}}:$ & Deposition velocity of a slurry, $(\mathrm{m} / \mathrm{s})$ \\
\hline $\mathrm{V}_{\mathrm{m}}:$ & Average velocity of a slurry, $(\mathrm{m} / \mathrm{s})$ \\
\hline $\mathrm{V}_{\mathrm{s}}$ : & Characteristic velocity scale, $(\mathrm{m} / \mathrm{s})$ \\
\hline v': & Velocity fluctuation in Y-direction, $(\mathrm{m} / \mathrm{s})$ \\
\hline w': & Velocity fluctuation in Z-direction, $(\mathrm{m} / \mathrm{s})$ \\
\hline$\alpha_{\mathrm{k}:}$ & Volume fraction of $\mathrm{k}^{\text {th }}$ phase \\
\hline$\beta:$ & Diameter ratio (ASME Nozzle) \\
\hline$\gamma:$ & Mean shear rate, $(1 / s)$ \\
\hline$\varepsilon:$ & Turbulent dissipation, $\left(\mathrm{m}^{2} / \mathrm{s}^{3}\right)$ \\
\hline$\varepsilon_{\mathrm{xy}}:$ & Shear strain, $(1 / \mathrm{s})$ \\
\hline$\eta_{\mathrm{r}}:$ & Relative viscosity of a slurry \\
\hline$\mu:$ & Dynamic viscosity, $(\mathrm{Pa}-\mathrm{s})$ \\
\hline$\mu_{\mathrm{m}}:$ & Dynamic viscosity of a slurry, (Pa-s) \\
\hline$\mu_{\mathrm{t}}$ & Turbulent viscosity, (Pa-s) \\
\hline
\end{tabular}




$\begin{array}{ll}\rho: & \text { Density of fluid, }\left(\mathrm{Kg} / \mathrm{m}^{3}\right) \\ \rho_{\mathrm{m}}: & \text { Density of slurry, }\left(\mathrm{Kg} / \mathrm{m}^{3}\right) \\ \tau_{\mathrm{ij}}: & \text { Reynolds stress tensor, }\left(\mathrm{N} / \mathrm{m}^{2}\right) \\ \varphi: & \text { Density of dispersed phase, }\left(\mathrm{Kg} / \mathrm{m}^{3}\right)\end{array}$




\section{ABBREVIATIONS}

$\begin{array}{ll}\text { ADP: } & \text { 2-Amino-4, 6-dimethyle Pyrimidine } \\ \text { APPI: } & \text { Atmospheric Pressure Photo Ionization } \\ \text { ASME: } & \text { American Society of Mechanical Engineers } \\ \text { CFD: } & \text { Computational Fluid Dynamics } \\ \text { DART: } & \text { Direct Analysis in Real Time } \\ \text { DNS: } & \text { Direct Numerical Simulation } \\ \text { FDA: } & \text { Food and Drug Administration } \\ \text { PIS: } & \text { Fourier Transform } \\ \text { MIR: } & \text { Middle Infrared } \\ \text { GUI: } & \text { Graphical User Interface } \\ \text { NIR: } & \text { Large Eddy Simulation } \\ & \end{array}$


RANS: $\quad$ Reynolds Averaging of Navier-Stokes

RDT: $\quad$ Rapid Distortion Theory

TOF: $\quad$ Time of Flight

VOF: $\quad$ Volume of Fluid 


\section{LIST OF TABLES}

Table Page

3.4.1. Maximum values of all the quantities required to calculate Stokes number ........42

3.5.1. Information with regards to the primary and secondary phase of the slurry ........47

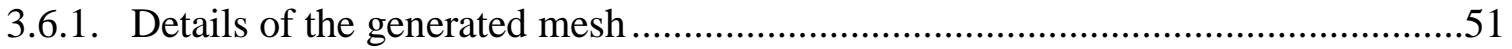

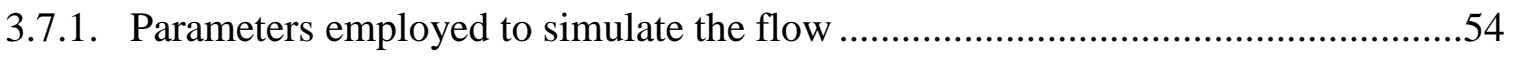

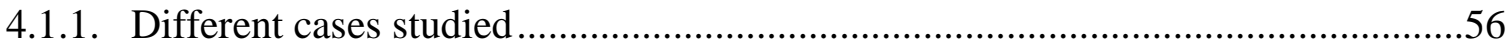




\section{LIST OF FIGURES}

Figure

Page

2.1.1. Pressure drop variation in $40 \mathrm{~mm}$ pipeline for fly ash slurry with flow velocity at different concentrations (\% by wt.) (Chandel et al. 2009) 8

2.1.2. Concentration profiles of $90 \mu \mathrm{m}$ particles at an efflux volumetric concentration of $\mathrm{C}_{\text {eflux }}=15 \%$ at fluid velocities (A) $\mathrm{V}=1.5 \mathrm{~m} / \mathrm{s}$ and (B) $\mathrm{V}=3.0 \mathrm{~m} / \mathrm{s}$ (Ekambara et al. 2009) .10

2.1.3. Illustration of particle suspension phenomena (Polaski et al. 2009) .11

2.1.4. Vertical concentration distribution of sand in a sand-water slurry flowing through a $51.5 \mathrm{~mm}$ pipeline (Roco and Shook 1984)

2.1.5. Vertical concentration distribution of sand in a sand-water slurry flowing through a $263 \mathrm{~mm}$ pipeline (Roco and Shook 1984)....

2.1.6. Computational validation of experimental results (sand-water slurry) of Roco and Shook (1984). Where (A) D=51.5 mm, V=3.78 m/s; (B) $\mathrm{D}=51.5 \mathrm{~mm}, \mathrm{~V}=4.33 \mathrm{~m} / \mathrm{s}$; (C) D=263 mm, V=3.5 m/s (Ekambara et al. 2009)

2.1.7. Measurement locations in concentric contraction test section (Wildman et al. 1992).....

2.1.8. Axial velocity profiles along the horizontal and vertical diameters of $5 \%$ silica gel slurry (Wildman et al. 1992).....

2.3.1. Schematic representing the functioning of a mass spectrometer (Clark 2000)

2.3.2. Schematic representing DART technique (Cho 2010) .20

2.4.1. The electromagnetic spectrum (Scarff et al. 2006) .23 
2.4.2. Principal features of near-infrared spectroscopy equipment (Blanco \& Villarroya 2002)

2.4.3. Qualification of major qualitative and quantitative multivariate analysis methods used in near-infra (NIR) spectroscopy (Blanco \& Villarroya 2003)

2.4.4. Diagrammatic representatation of different measurement approaches (Vaidyanathan et al. 1999)

3.2.1. Illustration of all the terms in the Navier-Stokes equation..................................33

3.3.1. Near-wall treatments (Hirsch 2007) …………..................................................

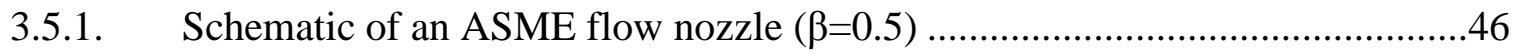

3.5.2. Schematic of the pipe section incorporated with an ASME flow nozzle.

3.6.1. An isometric view of the pipe-nozzle assembly ............................................48

3.6.2. Boundaries surrounding the control volume ...................................................51

4.1.1. Position of fully-developed region with respect to the exit plane of the nozzle (Represented by dark vertical lines) ......................................................57

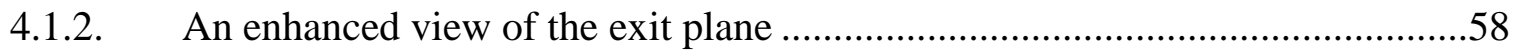

4.2.1. Fully-developed velocity profile of xylene plotted with power law profile

4.2.2. Numerically generated velocity profiles of $5 \%$ silica gel slurry

4.2.3. Comparison of velocity profiles of a sand-water slurry at a Reynolds number $\left(\mathrm{Re}_{\mathrm{D}}\right)$ of 300000

4.3.1.1. Concentration profiles of a xylene-ADP slurry $\left(\mathrm{d}_{\mathrm{p}}=38 \mu \mathrm{m}\right)$ at 0.25 efflux concentration computed with different cell volumes at $\left(\operatorname{Re}_{\mathrm{D}}\right.$ $=140000$ )

4.3.1.2. Turbulence kinetic energy profiles of a xylene-ADP slurry $\left(\mathrm{d}_{\mathrm{p}}=38\right.$ $\mu \mathrm{m})$ at 0.25 efflux concentration computed with different cell volumes at $\left(\operatorname{Re}_{\mathrm{D}}=140000\right)$

4.3.1.3. Concentration profiles of sand-water slurry at a $\mathrm{Re}_{\mathrm{D}}=300000$

4.3.1.4. Comparison of concentration profiles of sand-water slurry at initial volumetric concentration of 0.19 . .66

4.3.1.5. Comparison of concentration profiles of sand-water slurry at initial volumetric concentration of 0.24 . 
4.3.1.6. Comparison of concentration profiles of sand-water slurry at initial volumetric concentration of 0.29 .

4.3.1.7. Comparison of concentration profiles of sand-water slurry at initial volumetric concentration of 0.33 .

4.3.2.1. Concentration profiles of $38 \mu \mathrm{m}$ ADP particles at 0.05 initial efflux concentration as realized at the fully developed region and the exit plane

4.3.2.2. Concentration profiles of $38 \mu \mathrm{m}$ ADP particles at 0.2 initial efflux concentration as realized at the fully developed region and the exit plane

4.3.2.3. Concentration profiles of $38 \mu \mathrm{m}$ ADP particles at 0.3 initial efflux concentration as realized at the fully developed region and the exit plane

4.3.2.4. Concentration profiles of $75 \mu \mathrm{m}$ ADP particles at 0.05 initial efflux concentration as realized at the fully developed region and the exit plane

4.3.2.5. Concentration profiles of $75 \mu \mathrm{m}$ ADP particles at 0.2 initial efflux concentration as realized at the fully developed region and the exit plane

4.3.2.6. Concentration profiles of $75 \mu \mathrm{m}$ ADP particles at 0.3 initial efflux concentration as realized at the fully developed region and the exit plane

4.3.2.7. Concentration profiles of $150 \mu \mathrm{m}$ ADP particles at 0.05 initial efflux concentration as realized at the fully developed region and the exit plane

4.3.2.8. Concentration profiles of $150 \mu \mathrm{m}$ ADP particles at 0.2 initial efflux concentration as realized at the fully developed region and the exit plane

4.3.2.9. Concentration profiles of $150 \mu \mathrm{m}$ ADP particles at 0.3 initial efflux concentration as realized at the fully developed region and the exit plane

4.3.2.10. Velocity vectors of a 5 percent ADP-xylene slurry $\left(d_{p}=38 \mu \mathrm{m}\right)$ passing through the ASME flow nozzle at Reynolds number $\left(\operatorname{Re}_{\mathrm{D}}\right)$ of 140000 .75

4.3.2.11. Velocity contours at the mid-plane of a 5 percent ADP-xylene slurry $\left(\mathrm{d}_{\mathrm{p}}=38 \mu \mathrm{m}\right)$ passing through the ASME flow nozzle at a Reynolds number $\left(\operatorname{Re}_{\mathrm{D}}\right)$ of 140000 
4.3.2.12. Strain rate registered by the 5 percent ADP-xylene slurry along the axis of the ASME flow nozzle

4.3.3.1. Position of fully developed region with respect to the exit plane of the nozzle (Represented by dark vertical lines)

4.3.3.2. Concentration profiles of $38 \mu \mathrm{m}$ ADP particles at 0.05 initial efflux concentration as realized at the fully developed region and the exit plane

4.3.3.3. Concentration profiles of $38 \mu \mathrm{m}$ ADP particles at 0.2 initial efflux concentration as realized at the fully developed region and the exit plane

4.3.3.4. Concentration profiles of $38 \mu \mathrm{m}$ ADP particles at 0.3 initial efflux concentration as realized at the fully developed region and the exit plane

4.3.3.5. Concentration profiles of $75 \mu \mathrm{m}$ ADP particles at 0.05 initial efflux concentration as realized at the fully developed region and the exit plane

4.3.3.6. Concentration profiles of $75 \mu \mathrm{m}$ ADP particles at 0.2 initial efflux concentration as realized at the fully developed region and the exit plane

4.3.3.7. Concentration profiles of $75 \mu \mathrm{m}$ ADP particles at 0.3 initial efflux concentration as realized at the fully developed region and the exit plane

4.3.3.8. Concentration profiles of $150 \mu \mathrm{m}$ ADP particles at 0.05 initial efflux concentration as realized at the fully developed region and the exit plane

4.3.3.9. Concentration profiles of $150 \mu \mathrm{m}$ ADP particles at 0.2 initial efflux concentration as realized at the fully developed region and the exit plane

4.3.3.10. Concentration profiles of $150 \mu \mathrm{m}$ ADP particles at 0.3 initial efflux concentration as realized at the fully developed region and the exit plane

4.4.1. Comparison of mean strain rates along the axis of the ASME flow nozzle at two Reynolds numbers....

4.4.2. Comparison of turbulence intensities (I) at the fully developed region and the exit plan

4.4.3. Contours of turbulence intensity at the centre plane of the control volume 
4.4.4. Fully developed velocity profile of xylene at a Reynolds number of 140000

4.4.5. Velocity profile of xylene at the exit plane of the ASME flow nozzle at a Reynolds number of 140000.

4.4.1.1. Contours of volume fraction for a slurry (ADP-xylene) containing 38 $\mu \mathrm{m}$ ADP particles at an initial efflux concentration of 0.3 at $\operatorname{Re}_{\mathrm{D}}=140000$

4.4.1.2. Contours of volume fraction for a slurry (ADP-xylene) containing 75 $\mu \mathrm{m}$ ADP particles at an initial efflux concentration of 0.3 at $\operatorname{Re}_{\mathrm{D}}=140000$

4.4.1.3. Contours of volume fraction for a slurry (ADP-xylene) containing $150 \mu \mathrm{m}$ ADP particles at an initial efflux concentration of 0.3 at $\operatorname{Re}_{\mathrm{D}}=140000$.

4.4.1.4. Cross section contures of volume fraction at the fully developed region for a slurry (ADP-xylene) containing $150 \mu \mathrm{m}$ ADP particles at an initial efflux concentration of 0.3 at $\operatorname{Re}_{\mathrm{D}}=140000$.

4.4.1.5. Cross section contours of volume fraction at the exit plane for a slurry (ADP-xylene) containing $150 \mu \mathrm{m}$ ADP particles at an initial efflux concentration of 0.3 at $\operatorname{Re}_{D}=140000$

4.4.2.1. Contours of volume fraction for a slurry (ADP-xylene) containing 38 $\mu \mathrm{m}$ ADP particles at an initial efflux concentration of 0.3 at $\operatorname{Re}_{\mathrm{D}}=280000$

4.4.2.2. Contours of volume fraction for a slurry (ADP-xylene) containing 75 $\mu \mathrm{m}$ ADP particles at an initial efflux concentration of 0.3 at $\operatorname{Re}_{D}=280000$

4.4.2.3. Contours of volume fraction for a slurry (ADP-xylene) containing $150 \mu \mathrm{m}$ ADP particles at an initial efflux concentration of 0.3 at $\operatorname{Re}_{\mathrm{D}}=280000$

4.4.2.4. Cross section contours of volume fraction at the fully developed region for a slurry (ADP-xylene) containing $150 \mu \mathrm{m}$ ADP particles at an initial efflux concentration of 0.3 at $\mathrm{Re}_{\mathrm{D}}=280000$.

4.4.2.5. Cross section contours of volume fraction at the exit plane for a slurry (ADP-xylene) containing $150 \mu \mathrm{m}$ ADP particles at an initial efflux concentration of 0.3 at $\operatorname{Re}_{\mathrm{D}}=280000$ 


\section{CHAPTER I}

INTRODUCTION 


\subsection{Background}

Aspirin is one of the safest and least expensive analgesics available in the market. In America alone, close to $\$ 2$ billion are spent on non-prescription pain relievers, many of which contain aspirin. Although FDA (Food and Drug Administration) of America has voted against the use of aspirin for primary prevention of heart attack, there is an overwhelming portion of the scientific community that believes that aspirin reduces the risk of heart attack.

For a bottle of aspirin to reach our medicine cabinet from the manufacturing facility, it has to go through numerous steps that have to be controlled with a high degree of precision. Almost all the tablets available in the pharmacy are manufactured in batches. Before these tablets are transformed into a solid form, they are generally a combination of a liquid with solid particles suspended inside it. These types of mixtures are commonly known as slurries.

Slurry is a two-phase flow. By definition, a two-phase flow involves the simultaneous movement of two different phases, where the phase refers to the state of the matter (i.e. solid, liquid, or gas). During manufacturing, or processing of a pharmaceutical, most commonly, the two phases in question are: liquid which is referred to as the primary phase, and solid which is referred to as the disperse phase. The primary phase plays the role of the carrier while the disperse phase acts like a passenger.

While processing a pharmaceutical on a large scale, the process engineers deal with large volumes of these slurries, and they have to accommodate their transportation through pipelines in order to achieve the final product. While transporting normal fluids through pipelines is a challenge in itself, transporting slurries is a very different proposition.

One of the more crucial parameters that influence the transportation of a slurry through a pipe system is the concentration of the particles that are present in it. This will be dealt with in Section 
2.1 of Chapter 2. A design engineer has to account for the many parameters that affect the slurry that flows through the pipelines of a processing plant.

One of the more prominent roles of the process engineer is to determine the elemental composition of the slurry in question at every step of the manufacturing process so as to achieve the prescribed composition in the final product. They employ a wide array of techniques for the accomplishment of the aforementioned task. Near Infrared (NIR) spectroscopy and mass spectroscopy have proven to be among the most efficient of all the process monitoring techniques.

Mass spectroscopy is a technique that requires one to prepare a sample from the process stream (flowing slurry) before it can be presented to the spectrometer. This process is often time consuming, and sometimes impossible to realize in certain large scale manufacturing environments. Thus, the idea of performing mass spectral analysis of a flowing slurry, without having to prepare a sample is very attractive from a commercial point of view. This can provide a partial solution to the problem of meeting the growing demand for pharmaceuticals without compromising on their quality. Further, spectroscopic analysis of a flowing slurry promises to increase the efficiency of the whole pharmaceutical processing procedure.

Tackling a problem that pertains to a multi-disciplinary field requires a multi-disciplinary effort, so it becomes essential not only to understand the physics of the two-phase flow (slurry), but also the requirements of a mass spectrometer and the near-infrared spectrometer in relation to the slurry. In order to gain a thorough understanding of all the variables involved the subject of slurry flows and spectrometry (mass and near-infrared); they will be discussed in greater detail in the literature review section of this work. 


\subsection{Problem statement and presentation}

While performing the near infra-red spectral analysis of a slurry, the information relative to the concentration of the solid particles suspended in it is of supreme importance. The data generated by the near infra-red spectrometer can be believed to be credible only when the sample presented to it is representative of the total slurry volume. Concentration profiles are also crucial in case of mass spectroscopic analysis of a slurry. Kaushal and Tomita (2003) have demonstrated that by using an isokinetic probe, one can gain a wealth of information about the variation of concentration profiles of the solid particles in a slurry. Isokinetic sampling involves two steps. In the first step, a sample is withdrawn from the process stream. The second step requires one to perform density measurements on the collected samples, and determine the concentration of the solid particles. Thus, performing isokinetic sampling during the large scale manufacturing of a pharmaceutical causes unwanted delays. Thus, it is beneficial to have a fair idea of how the concentration profiles of the solid particles in slurry vary with respect to changes in field variables. Further, it is an added advantage if one could condition the concentration profiles in such a way that they are representative of the total slurry volume before a sample is delivered to the mass spectrometer for analysis.

\subsection{Objective}

Owing to the multi-disciplinary nature of the project proposal, this work has been bifurcated into two sections. At first glance, these two sections may seem completely segregated from one another, but in due course, the reader will realize that there is a concrete relation between them.

The first section which is the major section, presents the variation of the concentration profiles of the slurry in geometric configurations other than a straight cylindrical pipe. This section also makes an effort to predict suitable locations in the pipe flow where an ideal sample for mass spectroscopic analysis can be withdrawn, or be suitable for performing near-infrared 
spectroscopic analysis. These aforementioned geometric modifications are very easy to replicate in the experimental setup whose foundations are laid in the appendix section of this work.

The computational and experimental efforts presented in this work complement each other in a very unique manner. The variation of concentration profiles with respect to geometric changes for a slurry has rarely been documented. Thus, a computational analysis will provide guidance for design which must be verified by experiments.

The objective of this work is to investigate the variation of the concentration profiles of a particle-laden slurry at the exit plane of a rapid symmetric geometric contraction. The concentration profiles will be computationally predicted by varying the particle size $\left(\mathrm{d}_{\mathrm{p}}\right)$, initial efflux concentration $(C)$, and the flow Reynolds number $\left(\operatorname{Re}_{\mathrm{D}}\right)$. 
CHAPTER II

REVIEW OF LITERATURE 


\subsection{Slurry flows}

Since this work plans to address a technical problem pertaining to two loosely coupled disciplines (spectroscopy and fluid mechanics), one is forced to make a decision as to which of the fields must be described first. Being a mechanical engineer, an obvious decision of choosing to talk about slurry flows is made. Thus, in this particular section, some important parameters pertaining to slurry flows are discussed.

Transportation of a slurry from one point to another is one of the most challenging engineering tasks. This is because the hydraulic components present in the pipe system have to account not only for the carrier fluid but also the dispersed phase suspended in it. For instance, the pressure drop $\Delta \mathrm{p}(\mathrm{Kpa})$ realized in a pipe of length $1(\mathrm{~m})$ having a diameter of $\mathrm{D}(\mathrm{m})$ conveying a fluid of density $\rho\left(\mathrm{Kg} / \mathrm{m}^{3}\right)$ at an average velocity of $\mathrm{V}(\mathrm{m} / \mathrm{s})$ can be given as:

$$
\Delta p=f \cdot \frac{l}{D} \cdot \frac{\rho V^{2}}{2}
$$

To find the pressure drop when a slurry is being transported in similar conditions, equation (2.1.1) cannot be used. This is because the slurry is not a single phase flow. As mentioned previously, the slurry is comprised of two phases, and the presence of the dispersed phase (solid particles) prevents us from using the equation (2.1.1) as the concentration of the solid particles has not been accounted for.

Researchers have spent considerable amounts of time trying to understand the relation between the concentration of the solid particles present in a slurry and the pressure drop it registers. Good numbers of researchers including Chandel et al. (2009) agree that the pressure drop in slurry pipelines increases with particle concentration. Chandel et al. experimented with fly ash slurries, and Fig.2.1.1 presents their findings. 


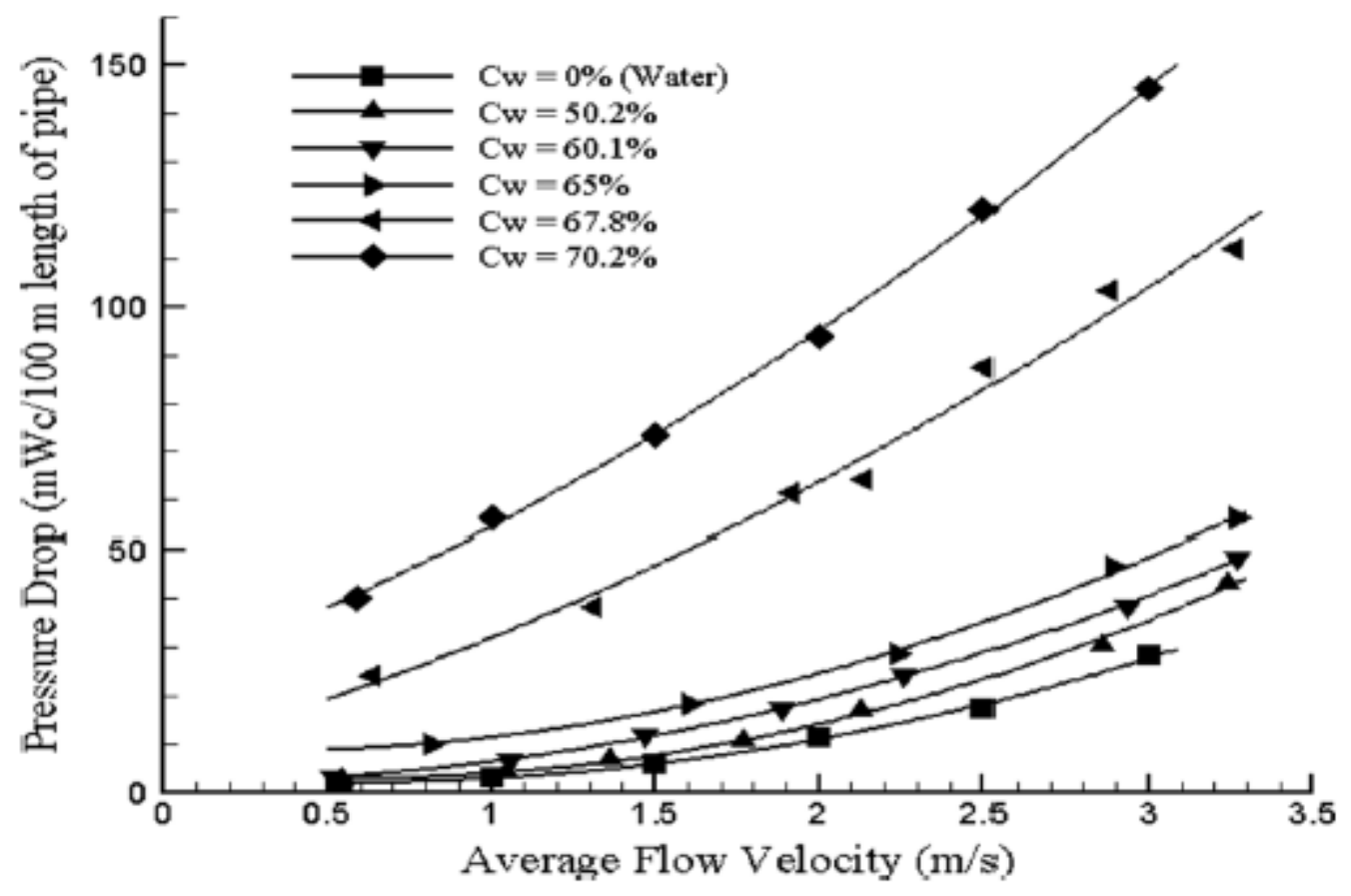

Figure 2.1.1: Pressure drop variation in $40 \mathrm{~mm}$ pipeline for fly ash slurry with flow velocity at different concentrations (\% by wt.) (Chandel et al. 2009)

Stickel et al. (2005) stated that suspensions of solid particles in carrier fluids are a class of complex fluids, and can be further differentiated according to the physical and chemical nature of the suspended particles and carrier fluids. Through their extensive experimental research they were able to conclude that highly concentrated slurries are dominated by multiple-body interactions, and their rheology is mostly non-Newtonian.

Relative viscosity $\left(\eta_{\mathrm{r}}\right)$, which is the ratio of the viscosity of the slurry to the viscosity of the primary phase, is another parameter that has to be taken into consideration while working with slurries. The relative viscosity $\left(\eta_{\mathrm{r}}\right)$ in turn depends heavily on the concentration of the solid particles (disperse phase). According to Stickel at al. (2005), the relative viscosity of a slurry is a function of concentration of the solid particles $\varphi$, Reynolds number of the flow $\operatorname{Re}_{\gamma}$, and Peclet 
number $\mathrm{Pe}_{\gamma}$. In all the following equations the physical quantities represented by a subscript ' 0 ' are for the carrying fluid or the continuous phase of the slurry. (kT) represents the thermal energy, and $\gamma$ is the shear rate.

$$
\begin{gathered}
\eta_{\gamma}=f\left(\phi, P e_{\gamma}, \operatorname{Re}_{\gamma}\right) \\
\eta_{r}=\frac{\eta}{\eta_{0}} \\
\rho_{r}=\frac{\rho}{\rho_{r}} \\
\phi=\frac{4 \pi}{3} n a^{3} \\
P e_{\gamma}=\frac{6 \pi \eta_{0} a^{3} \gamma}{k T} \\
\operatorname{Re}_{\gamma}=\frac{\rho_{0} a^{2} \gamma}{\eta_{0}}
\end{gathered}
$$

Chang \& Powell (2002) assumed that at high particle concentrations i.e., at $\varphi>0.5$ the Reynolds number $\operatorname{Re}_{\gamma}$ and the Peclet number $\mathrm{Pe}_{\gamma}$ can be neglected, and hence the relative viscosity $\eta_{\mathrm{r}}$ depends solely on the concentration of the solid particles $\varphi$.

$$
\eta_{\gamma}=f(\phi)
$$

The concentration profiles for a slurry are similar to the velocity profile of a fluid flowing through a pipe. The profile represents the variation of the particle concentration along the vertical diameter of a horizontal pipe. Ekambara et al. (2009) computationally validated Schaan et al.'s 
(2000) experimental concentration profiles of $90 \mu \mathrm{m}$ sand particles. Their plots are presented in

Fig.2.1.2.
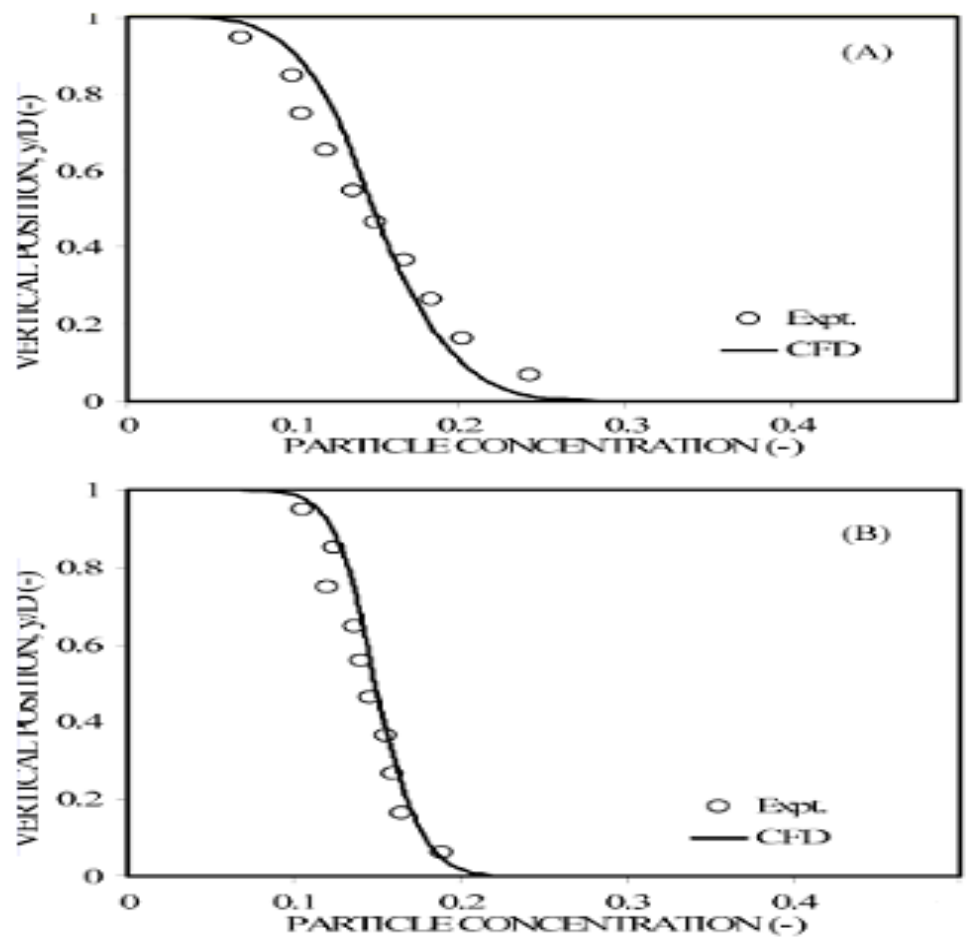

Figure 2.1.2: Concentration profiles of $90 \mu \mathrm{m}$ particles at an efflux volumetric concentration of $C=15 \%$ at fluid velocities (A) $V=1.5 \mathrm{~m} / \mathrm{s}$ and $(B) \mathrm{V}=3.0 \mathrm{~m} / \mathrm{s}$ (Ekambara et al. 2009)

By observing the curves in Fig. 2.1.2, one can gain a basic understanding about the parameters that affect the concentration profiles. Both the curves indicate that the concentration by volume (C) of the particles is higher at the bottom of the pipe than at any other position. This clearly suggests that the concentration profiles depend on the mass and volume of the suspended particles. While comparing the profiles at $\mathrm{V}=1.5 \mathrm{~m} / \mathrm{s}$ and $\mathrm{V}=3 \mathrm{~m} / \mathrm{s}$, one can notice that higher velocities tend to reduce the concentration of the particles at the bottom of the pipe, and distribute it evenly. At this juncture, it is best to digress from concentration profiles, and discuss about the deposition velocity $\left(\mathrm{V}_{\mathrm{d}}\right)$ of a particle-laden slurry. 
Intuitively, in order to suspend particles in a fluid, the momentum acting in the vertical direction should at least compensate for the particle buoyant mass if not exceed it. The only thing that can provide the suspended particles with this momentum is the turbulent eddy drag force. Thus, the fluid should flow in the pipe at a Reynolds number that generates the level of turbulence to keep the particles in suspension. For constant fluid rheological parameters, size of the particles $\left(\mathrm{d}_{\mathrm{p}}\right)$, and pipe diameter $(\mathrm{D})$, the deposition velocity $\left(\mathrm{V}_{\mathrm{d}}\right)$ can be defined as the mean velocity of the continuous phase at which the particles start to settle at the bottom of the pipe under the action of gravity. Figure 2.1.3 gives an insightful illustration of the whole process.

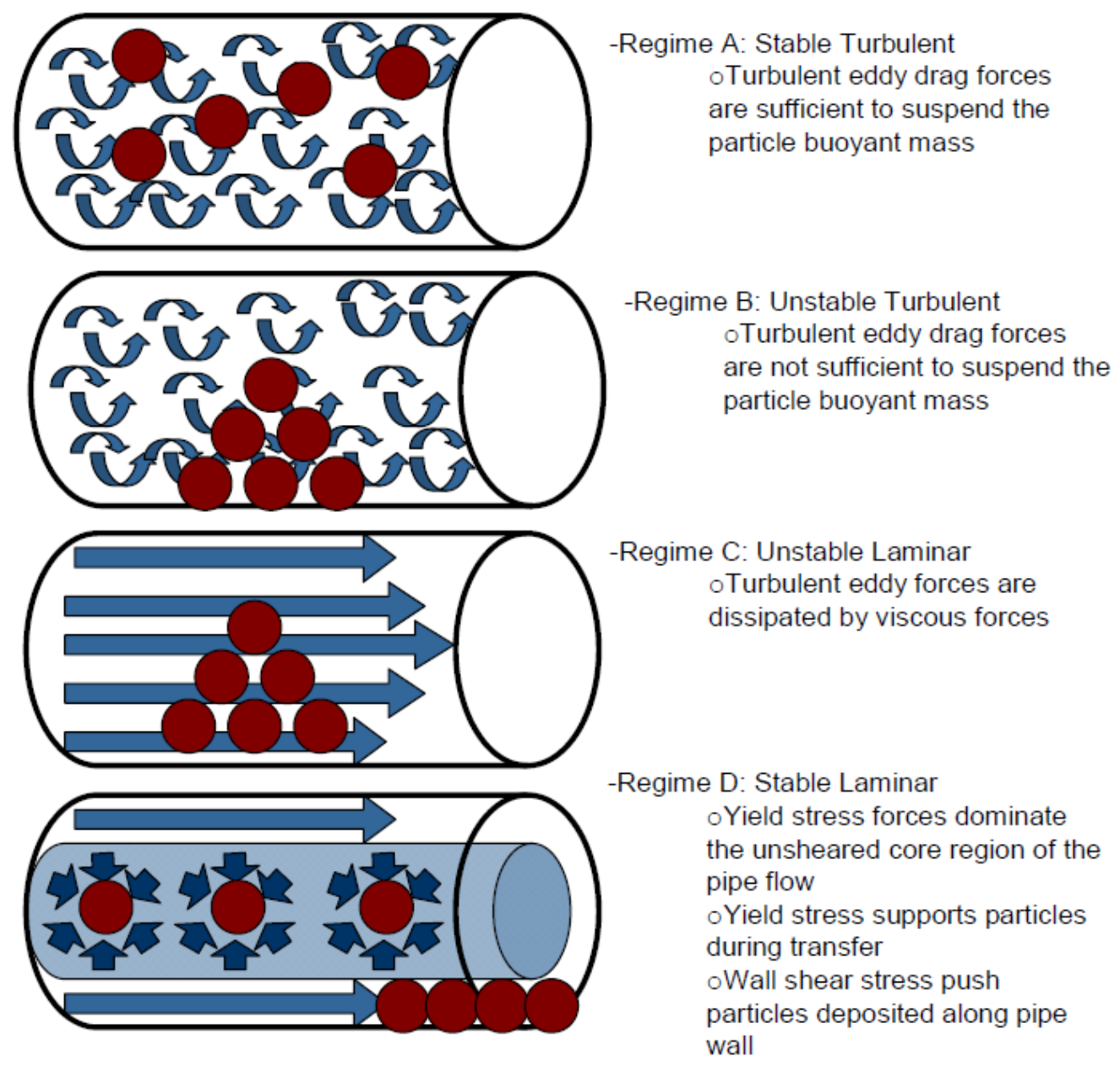

Figure 2.1.3: Illustration of particle suspension phenomena (Polaski et al. 2009) 
Resuming the discussion on concentration profiles, it is worth mentioning at this point that the concentration variation in relation to changes in the type of fluid, particle diameter $\left(\mathrm{d}_{\mathrm{p}}\right)$, and different pipe diameters (D) has been extensively dealt with by Roco and Shook (1984), Schaan et al. (2000), Gillies and Shook (2000), Gillies et al. (2004), and Kaushal and Tomita (2007). Roco and Shook (1984) performed a thorough experimental analysis by varying all the parameters that were mentioned previously, and derived empirical relations that would predict the variation of concentration profiles. Their findings are presented in Fig. 2.1.4 and Fig. 2.1.5.

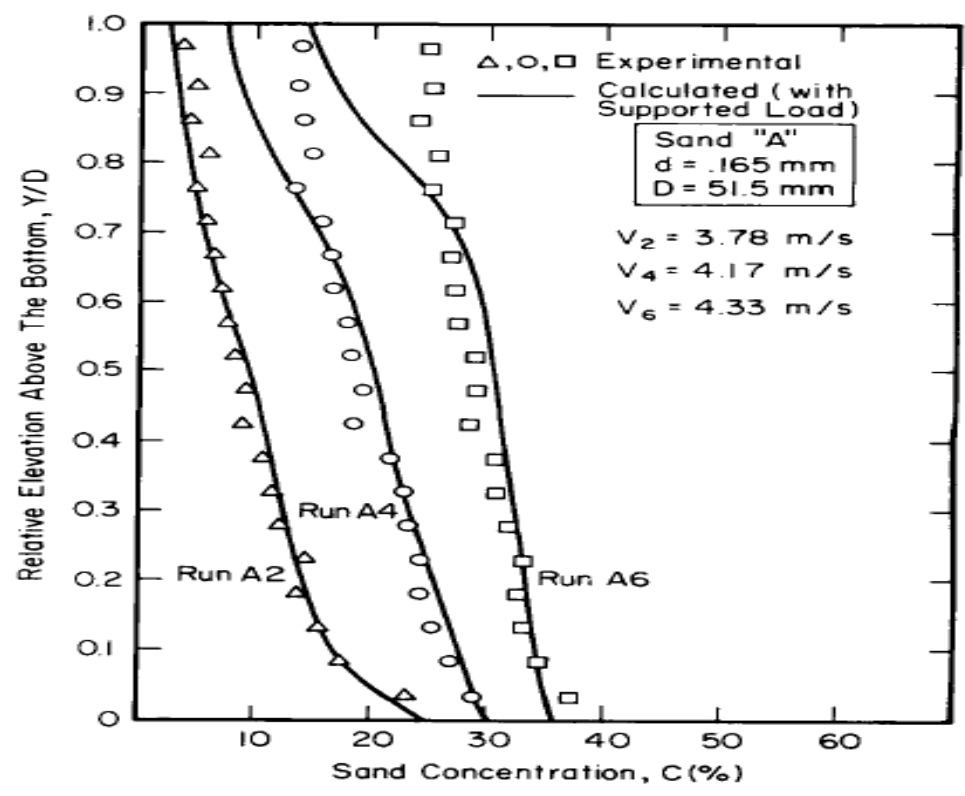

Figure 2.1.4: Vertical concentration distribution of sand in a sand-water slurry flowing through a $51.5 \mathrm{~mm}$ pipeline (Roco and Shook 1984) 


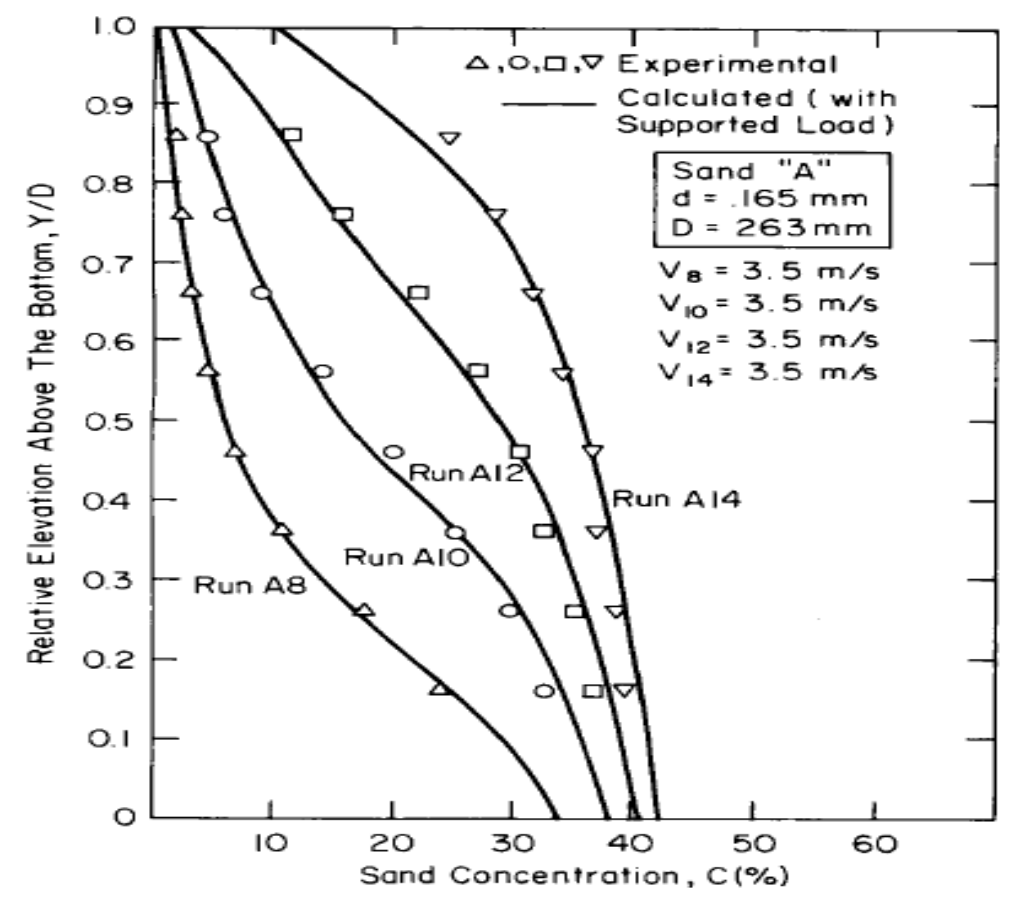

\section{Figure 2.1.5: Vertical concentration distribution of sand in a sand-water slurry flowing through a $263 \mathrm{~mm}$ pipeline (Roco and Shook 1984)}

Roco and Shook (1984) proposed the concept of "supported load". This term defines the weight of the fraction of the solid particles which is supported by Coulombic contact. Coulumbic contact can be understood by visualizing a pipe with particles where the fluid is at rest. In this state, all the particles settle at the bottom of the pipe. Thus, the weight of the particles is supported by the reaction from the wall, or from neighboring particles. Roco and Shook conclude that the concept of "supported load" plays a crucial role in the computation of concentration and velocity distribution of larger particles. Recently, the experimental and empirical results of Roco and Shook (1984) were validated computationally by Ekambara et al. (2009). Ekambara et al. used ANSYS-CFX computational fluid dynamic (CFD) package to achieve the results that are presented in Fig. 2.1.6. 

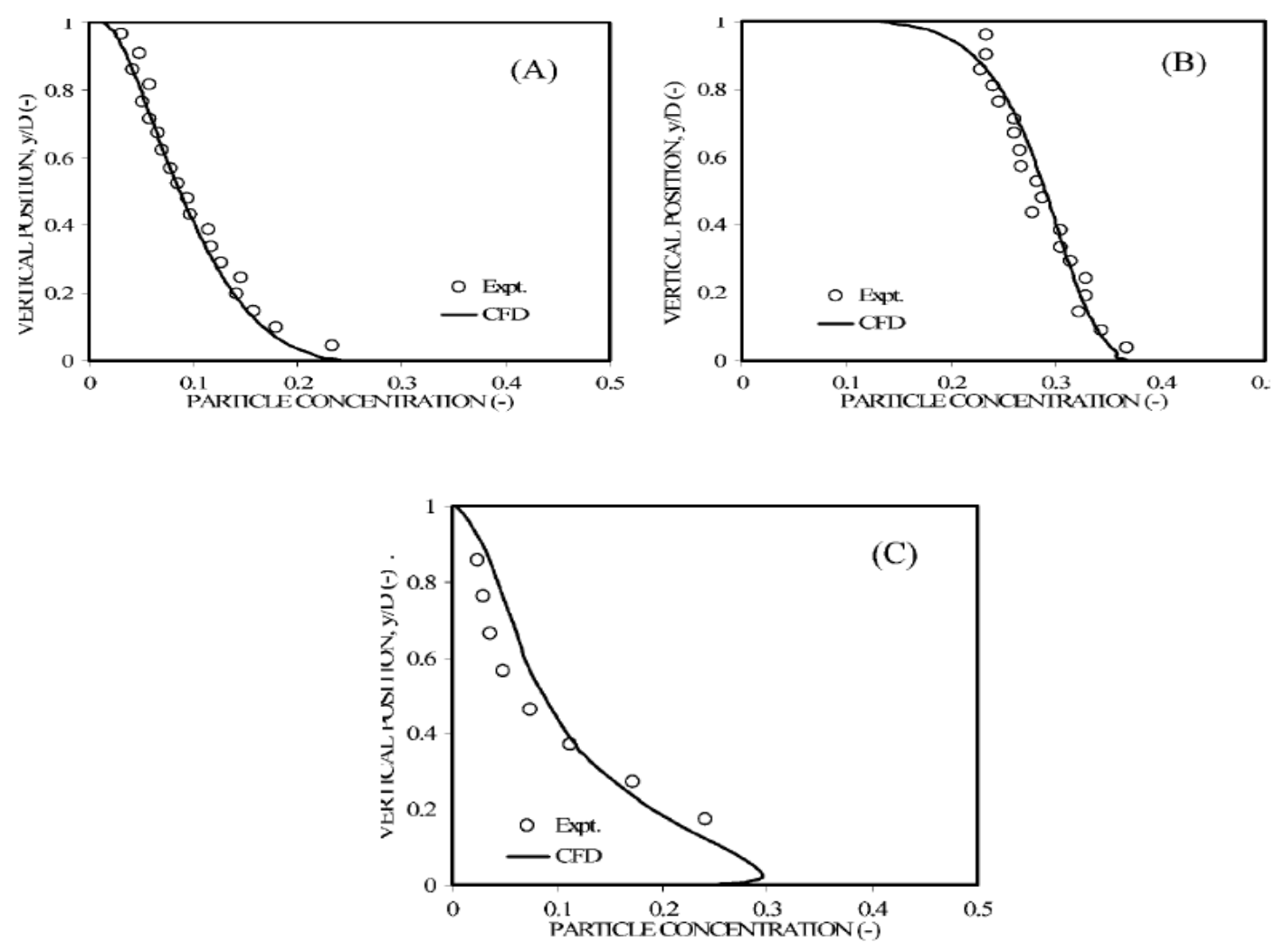

Figure 2.1.6: Computational validation of experimental results (sand-water slurry) of Roco and Shook (1984). Where (A) $D=51.5 \mathrm{~mm}, V=3.78 \mathrm{~m} / \mathrm{s}$; (B) $D=51.5 \mathrm{~mm}, V=4.33 \mathrm{~m} / \mathrm{s}$; (C) $\mathrm{D}=263 \mathrm{~mm}, \mathrm{~V}=3.5 \mathrm{~m} / \mathrm{s}$ (Ekambara et al. 2009)

Thus, one can clearly observe that computational fluid dynamics can be a good predictor of concentration profiles of the solid particles present in a slurry.

I have been unable to find documented accounts where variation of concentration profiles when a slurry is made to flow through a geometric contraction were studied. However, velocity profiles of a slurry flowing through a contraction were studied by Wildman et al. (1992) who used 5\% silica gel as their working fluid. Their results are presented in Fig. 2.1.7 and Fig. 2.1.8. 


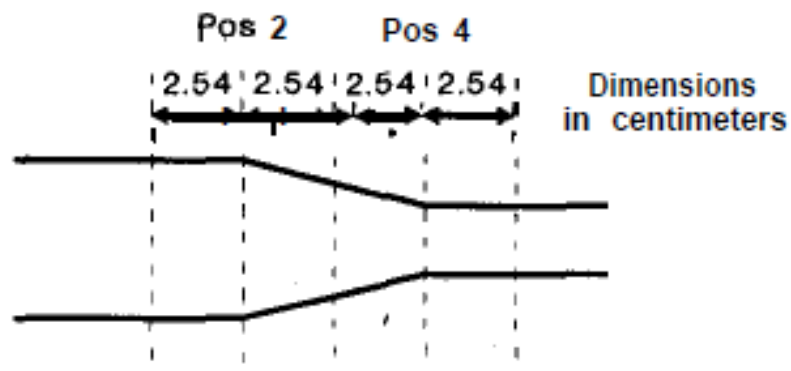

Pos 1 Pos 3 Pos 5

Figure 2.1.7: Measurement locations in concentric contraction test section (Wildman et al. 1992)

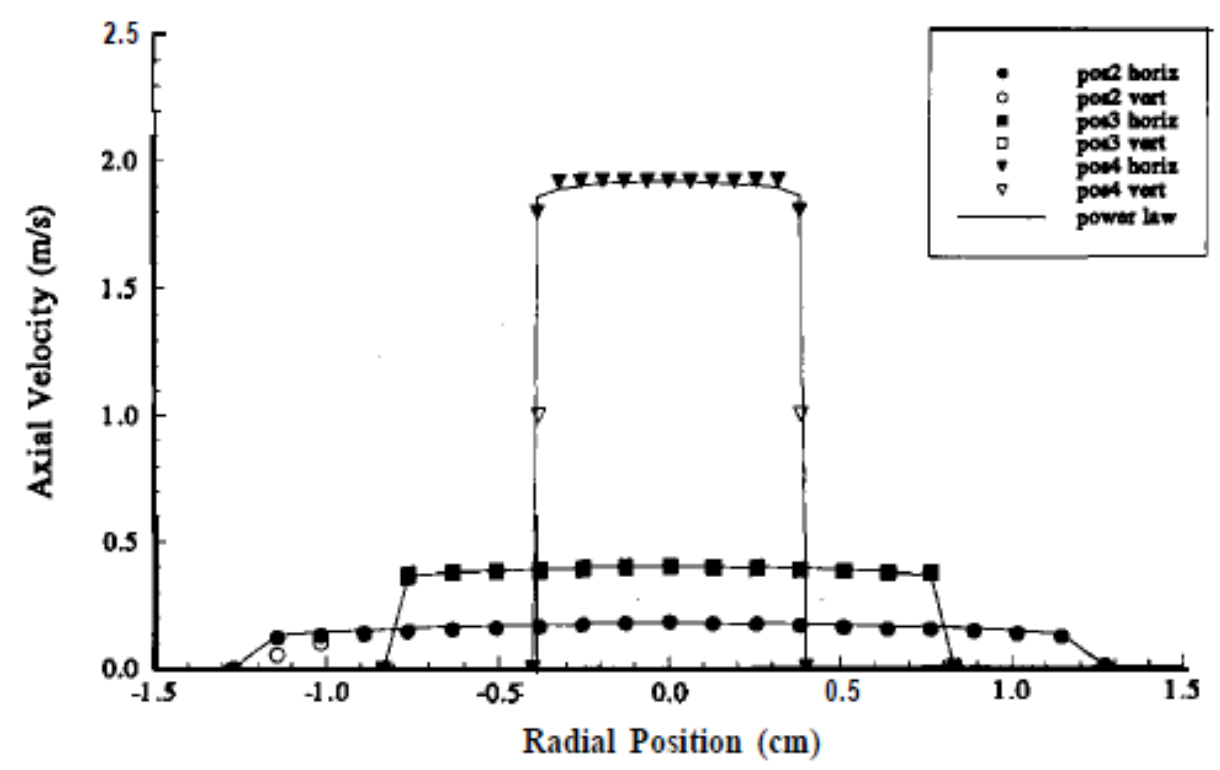

Figure 2.1.8: Axial velocity profiles along the horizontal and vertical diameters of $5 \%$ silica gel slurry (Wildman et al. 1992)

Wildman et al. conclude that the achieved experimental results were in good agreement with theoretical predictions of Hanks and Dadia (1971). Hanks and Dadia (1971) expended commendable effort in order to develop semi-theoretical relations for turbulent flow of Newtonian fluids in smooth pipes.

Chen et al. (2008) presents a rigorous analysis on the pressure drop variation in slurries when they are conveyed through suddenly contracting rectangular ducts. A religious search through 
literature revealed that the variation of concentration profiles of a slurry when conveyed through contractions has hardly been documented. This is a suitable endeavor to undertake as it may provide us with ways to "program" the concentration profiles of slurry in a way that is compatible with the kind of sample that a near infra-red spectrometer and a mass spectrometer demand. The subject of mass spectroscopy is dealt with finer detail in Section 2.3 of this work. Before taking a leap into the realm of mass spectroscopy, there is an interesting flow phenomenon that goes by the name of Rapid Distortion Theory (RTD) that requires due attention. What follows next is a concise introduction to the theory of rapid distortion.

\subsection{Flow of a fluid through a symmetric contraction}

The flow of a fluid through a geometric contraction has attracted the attention of numerous researchers over the years. This interest stems from the fact that in this particular case, turbulent flows display characteristics that are uncharacteristic of turbulence. According to Libby (1996), a turbulent flow through a rapid contraction is characterized by an enormous increase in mean shear rate in the main flow direction. This results in weak turbulence levels, and renders usual turbulent mechanisms passive. This phenomenon was first observed by G. I. Taylor while he was trying to find ways to suppress wind tunnel turbulence. This phenomenon was named 'Rapid Distortion Theory' (RDT).

According to Savill (1987), turbulence contains a range of scales and associates these with ranges of wave numbers. The large scale, low-wave number eddies interact with the mean flow and gain energy from it. Simultaneously, these eddies get distorted, and result in the manifestation of Reynolds stresses. The energy of these eddies cascades to small scales, where it is dissipated to viscosity. The aforementioned lines suitably summarize the mechanism of turbulence. 
An understanding of the mechanism of turbulence aids to capture the essence of rapid distortion theory. A preliminary, yet comprehensive study of rapid distortion theory was conducted by Batchelor and Proudman (1954) who are considered its pioneers. According to Batchelor and Proudman, when the turbulent fluid is subjected to a superimposed uniform distortion (symmetric geometric contraction), a change in the relative position of fluid particles due to turbulence is negligible. In addition, relative changes in energies of 3 velocity components are found to be independent of the properties of turbulence. Thus, the effect of a large symmetrical contraction on the turbulent flow field can be summarized by stating that the vorticity of the flow field is parallel to the axis of the geometric contraction. It has to be noted that for pure rapid distortion to take place, the axial mean velocity of the fluid exiting the contraction has to be at least 5 times greater than its entrance velocity (Libby 1996). For a two phase flow, achieving the previously stated constraint may result in complications such as the clogging of the contracting section. Hence, all the cases in this study are simulated such that the axial mean velocity at the exit of the contraction is at least 4 times that of the mean axial entrance velocity. Thus, the simulated flow field may be considered as close to rapid distortion conditions as practically possible.

There is enough evidence present in the literature that suggests that symmetric geometric contractions tend to have a tranquilizing effect on the turbulent flow field, hence making the velocity profiles more uniform, and reducing axial turbulence fluctuations. Whether this effect is similar in relation to the concentration profiles of solid particles in a two-phase flow is a question that has hardly received any attention. Since the variation of concentration profiles of a slurry play a pivotal role in both mass spectroscopy and near infra-red spectroscopy of a two-phase flow, the present works tries to answer this question by employing a computational fluid dynamic (CFD) analysis.

We as mechanical engineers are faced with an arduous task of presenting a sample from a region that is highly chaotic to a sensitive device like the mass spectrometer. If not before, 
definitely at this point, it becomes rather evident that this work is part of a proposal that encompasses a wide range of disciplines. Even though the fundamentals of mass spectroscopy may seem esoteric to a mechanical engineer, this work is not complete without a thorough discussion of the aforementioned field.

\subsection{Mass spectroscopy}

The inception of mass spectroscopy dates back a century. It was Eugen Goldstein (1858-1930), in 1886, who observed rays (streams of particles) in gas discharges, under low pressures, that travelled from an anode (positively charged electrode). He named them anode rays. Later, Wilhelm Wien (1864-1928) proved that the positively charged stream of particles (anode rays) could be separated according to their charge (q) to mass (m) ratio (q/m) (Boggess 2001). The configurations of mass spectrometers may have undergone a change over the years, but the underlying principle of operation is still the same.

The working of the mass spectrometer can be subdivided into 4 stages. They are explained as below.

Ionization: A sample of the material in question is presented to the mass spectrometer. The atoms of the material are ionized to form positive ions.

Acceleration: The ions are accelerated so that they all have some kinetic energy.

Deflection: Ions are deflected by magnetic fields. The lighter they are the more easily they get deflected. The amount of deflection also depends on how much charge the ion carries.

Detection: The beam of ions is detected by a detector. The ions with smaller $(\mathrm{q} / \mathrm{m})$ ratio reach the detector faster. The detector displays a graph that has the $(\mathrm{q} / \mathrm{m})$ ratio on the horizontal axis and number of particles per second on the vertical. This graph is representative of the mass of the elements present in the sample.

Figure 2.3.1 sheds more light on the different stages discussed. 


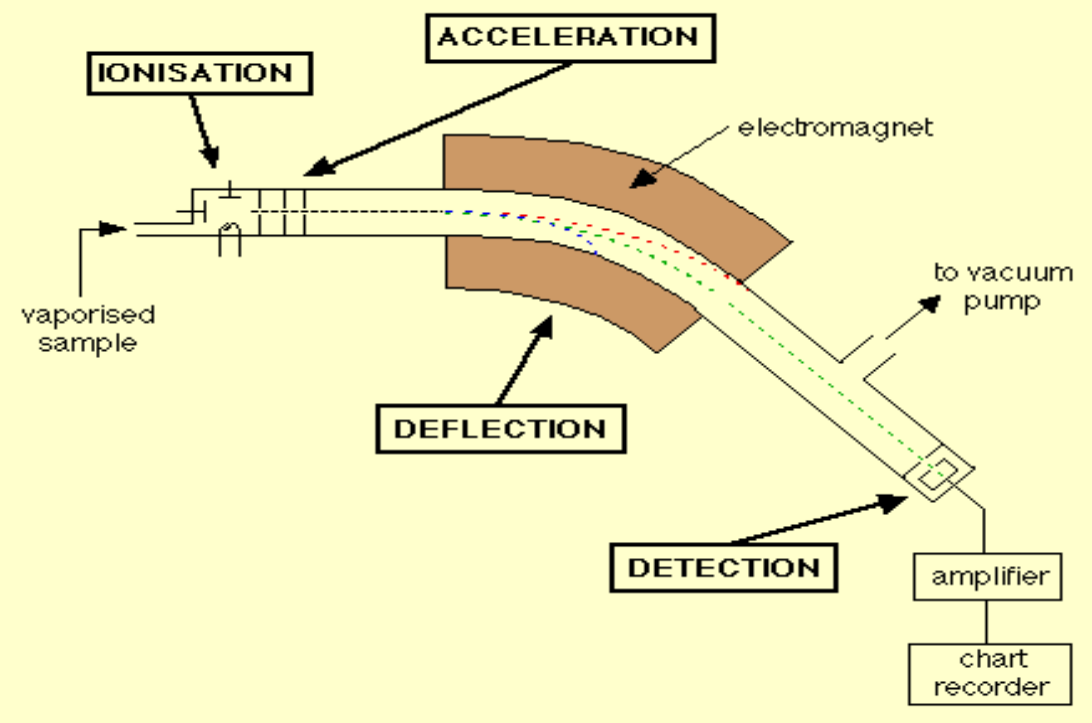

Figure 2.3.1: Schematic representing the functioning of a mass spectrometer (Clark 2000)

Mass spectroscopy has come a long way since it was first used to analyze amino acids and peptides in 1958. Rapid advancements in detector, optical probe, and computer technology have propelled the field of mass spectroscopy into a new era of innovation. A range of different methods to achieve ionization, acceleration, deflection, and detection have surfaced, predominantly because of the increase in the diversity of mass spectroscopic applications. The methods that hold great potential for their application in sampling of slurries are dealt with in the following paragraphs.

Fenn et al. (1989) generated spectra for biopolymers like oligonucleotides that possess molecular weights of the order of 130,000 a.m.u using an innovative technique called Electro spray ionization (EPI). This method can be particularly suitable for analyzing slurry samples as pharmaceutical slurries generally contain long-chain organic molecules that have high molecular weights. Atmospheric pressure photo ionization (APPI) is another method that could be employed for analyzing slurries. This approach utilizes a discharge lamp that generates ultra-violet photons. These photons are absorbed by the sample in question, and ionize the molecules present in it. The process of ionization takes place at atmospheric pressure. Robb et al. (2000) has successfully 
demonstrated the use of APPI for analyzing samples. However, documented accounts of experiments where the aforementioned techniques were utilized to analyze slurry flows are nonexistent.

Recently, an innovative technique where a jet of excited-state nitrogen gas is used to impact the surface of the sample to generate ions has been developed. This technique is named Direct Analysis in Real Time (DART) (Cody et al. 2005). The incorporation of this technique to sample slurries seems plausible after one observes Fig. 2.3.2, an approach assembled by Cho (2010).

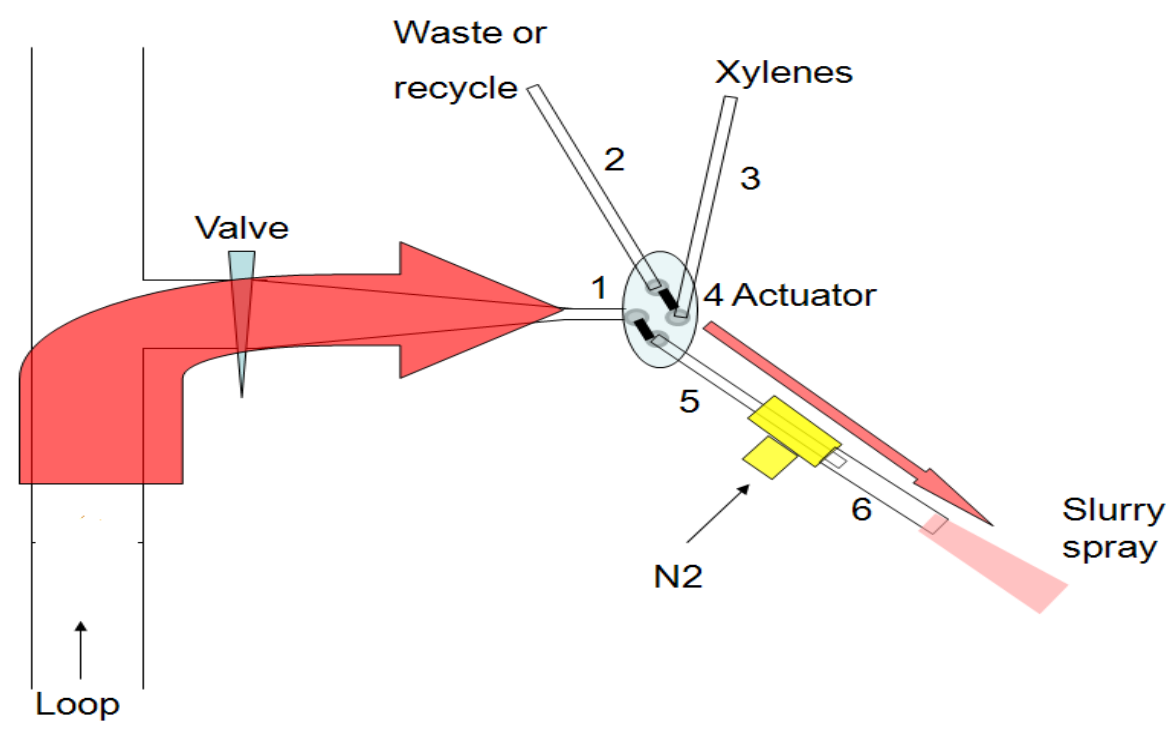

Figure 2.3.2: Schematic representing "DART" technique (Cho 2010)

According to Cody et al. (2005), the aforementioned technique can be used in coordination with high resolution time-of-flight (TOF) type of mass spectrometers to increase their selectivity while discerning elemental compositions of samples. However, it has been rarely documented that time-of-flight (TOF) analyzers possess the precision necessary for quantitative analysis. Thus, this hinders their usage for slurry analysis.

Going back to Fig 2.3.2, one can observe that the slurry is being drawn from the process stream, converted into a spray, and then delivered to a mass spectrometer (not seen in the figure). It is highly imperative that the volume of the slurry drawn from the flow should be representative 
of total slurry volume. Being more specific, if the total slurry volume has $\mathrm{X} \%$ of particles by weight or volume, the sample drawn should also possess a volumetric percentage or weight percentage of particles that is very close to $\mathrm{X} \%$. As discussed previously in Section 2.1, gravity produces a concentration gradient along the vertical diameter of a horizontal pipe. A sample drawn from this section is bound to have a concentration that is not representative of the total slurry volume. Thus, the underlying solution to this problem is to somehow condition the concentration profiles at the sampling location to be as uniform as practically possible. It has been discussed in Section 2.2 that the velocity profiles of a fluid exiting a geometric contraction display a high degree of uniformity. So pronounced is the extent of their uniformity that they are referred to as "top hat" profiles. Thus, it is fair to assume that even the concentration profiles may display a similar character after exiting a contraction, after all, it was stated earlier that concentration profiles were strong functions of fluid velocity. Availability of state-of-the art computational fluid dynamic (CFD) software would inspire any fluid mechanics engineer to put this theory to practice.

While mass spectroscopy is a technique that takes the sample to the detection source, there is a different breed of analysis techniques that take the detection source to the sample. Near-infrared (NIR) spectroscopy is one such technique. What makes near-infrared spectroscopy a potential agent to analyze slurry flows can be understood by going through Section 2.4.

\subsection{Near infra-red (NIR) spectroscopy}

This particular section of the document describes, in great detail, near infra-red (NIR) spectroscopy, and how it is used as a tool of measurement in various industries, particularly the pharmaceutical industry. Near infra-red spectroscopy involves the conditioning of the signal with mathematical tools. These mathematical tools will be discussed, but only to the extent that the reader gets a basic idea of the concepts. Near infra-red spectroscopy is used in different kinds of analysis: qualitative and quantitative. It is also used in different types of measurements: on-line, 
at-line, and off-line to name a few. A brief description of these analysis and, measurement techniques is also presented in this section.

The near infra-red spectrum was the first non-visible radiation discovered in the absorption spectrum. It spans the wave length range $700-2500 \mathrm{~nm}$, in which absorption bands correspond mainly to overtones of fundamental vibrations (Blanco et al. 1998). Its occurrence in the electromagnetic spectrum is illustrated in Fig 2.4.1. Although it was discovered in the 1800s, its mainstream usage was in a state of dormancy almost until the mid 1950s. Thus, its sedated condition prompted the scientific community to deem it as: "sleeper among spectroscopic techniques" (Blanco et al). But little did they know; its true potential was just waiting to be discovered. According to Blanco and Villarroya (2002), it was in the 1970s that near infra-red spectroscopy was first used to analyze agricultural and food samples. However, the pharmaceutical industry was slow to adapt this technology as it lacks the ability of mid infra-red (MIR) spectroscopy to analyze samples by mere inspection of their spectra. But rapid advancements in fiber-optic, detector, and micro computing technology helped researchers realize that the dependence of near infra-red spectra on both the chemical composition and physical properties of the samples in question was a characteristic that could be exploited. This innate quality of near infra-red spectral signal when treated with powerful mathematical tools can not only help in identifying the compounds present in the sample (qualitative), but at the same time furnish enough information to discern non-chemical parameters (quantitative). Thus, considering near infra-red spectroscopy as a "sleeper" among the spectroscopy techniques is definitely not the opinion of the present scientific community. 


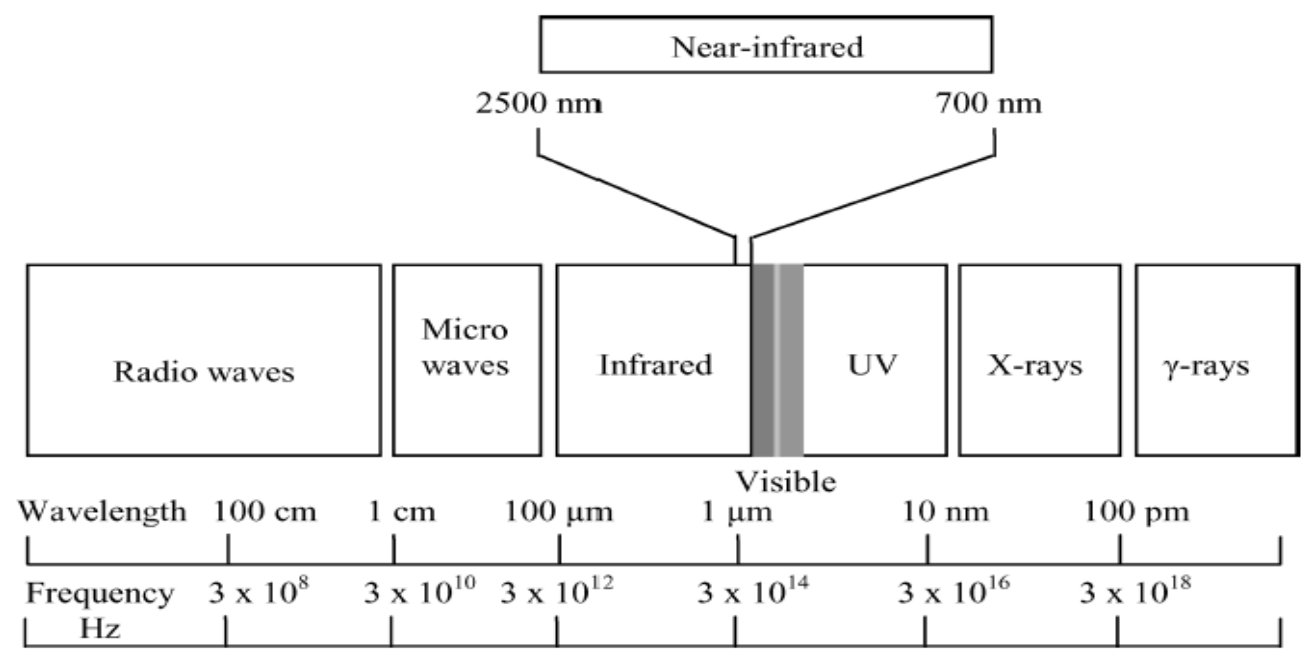

Figure 2.4.1: The electromagnetic spectrum (Scarff et al. 2006)

The most elementary capability of near infra-red spectroscopy is to identify the functional groups such as $(\mathrm{C}-\mathrm{H}, \mathrm{O}-\mathrm{H}, \mathrm{N}-\mathrm{H}$, and $\mathrm{S}-\mathrm{H})$ present in a compound, eventually determining the structure of a compound. The atoms in these functional groups are bonded together with covalent bonds. The structure of the functional group can be visualized as two spheres connected with the help of a vibrating spring. The frequency of the vibrating spring (covalent bond) is dependent on the masses of the atoms, and also on the strength of the bond. When acted upon by radiation, the bonds absorb energy, and change their frequency of vibration. The functional groups mentioned earlier, absorb radiation in a particular frequency range, and a change in the frequency of their bond vibrations is helpful in identifying the functional groups. The aforementioned processes take place inside a box that is as small as a cockpit voice recorder. This is called a spectrometer.

Near-infrared spectrometers are of two distinct types: Fourier Transform (FT-NIR) and dispersive. The arrangement of the components inside a Fourier Transform near infra-red (FTNIR) spectrometer can be simplified to understand the basic principle of its operation. The near infra-red source, guiding mirrors, interferometer, and the detector are arranged in series. The sample under investigation is placed at a convenient position between the source and the detector. 
The source emits the near infra-red radiations, and these radiations reach the sample after passing through the interferometer. The interferometer is a device that generates what is known as an interferogram. An interferogram is a type of a graph that holds the information of all the frequencies that were emitted. The interferogram undergoes changes once it passes through the sample. The computer with the decoding software which is connected to the detector, performs a Fourier transform on the interferogram, and decodes it. The software also plots a graph that provides a relation between the percentages of radiation transmitted against the wave number $\left(\mathrm{cm}^{-1}\right)$. This graph is interpreted by the chemist who eventually comes up with the different functional groups present in the sample.

A dispersive type of a near-infrared spectrometer employs a prism or a grating monochromator that facilitates the separation of visible light into its frequencies. These types of spectrometers have the capability to measure a small part of the spectrum at any given time; therefore the spectrum is scanned by moving the grating, mirrors or the detector. Thus, subtle movements in optical components caused by changes in the surrounding temperature can drastically affect the measurement of absorbance at each wavelength. This results in discrepancies while developing multivariate calibration models (Scarff et al. 2006). The principal features of near-infrared spectroscopy equipment are provided in Fig 2.4.2. 


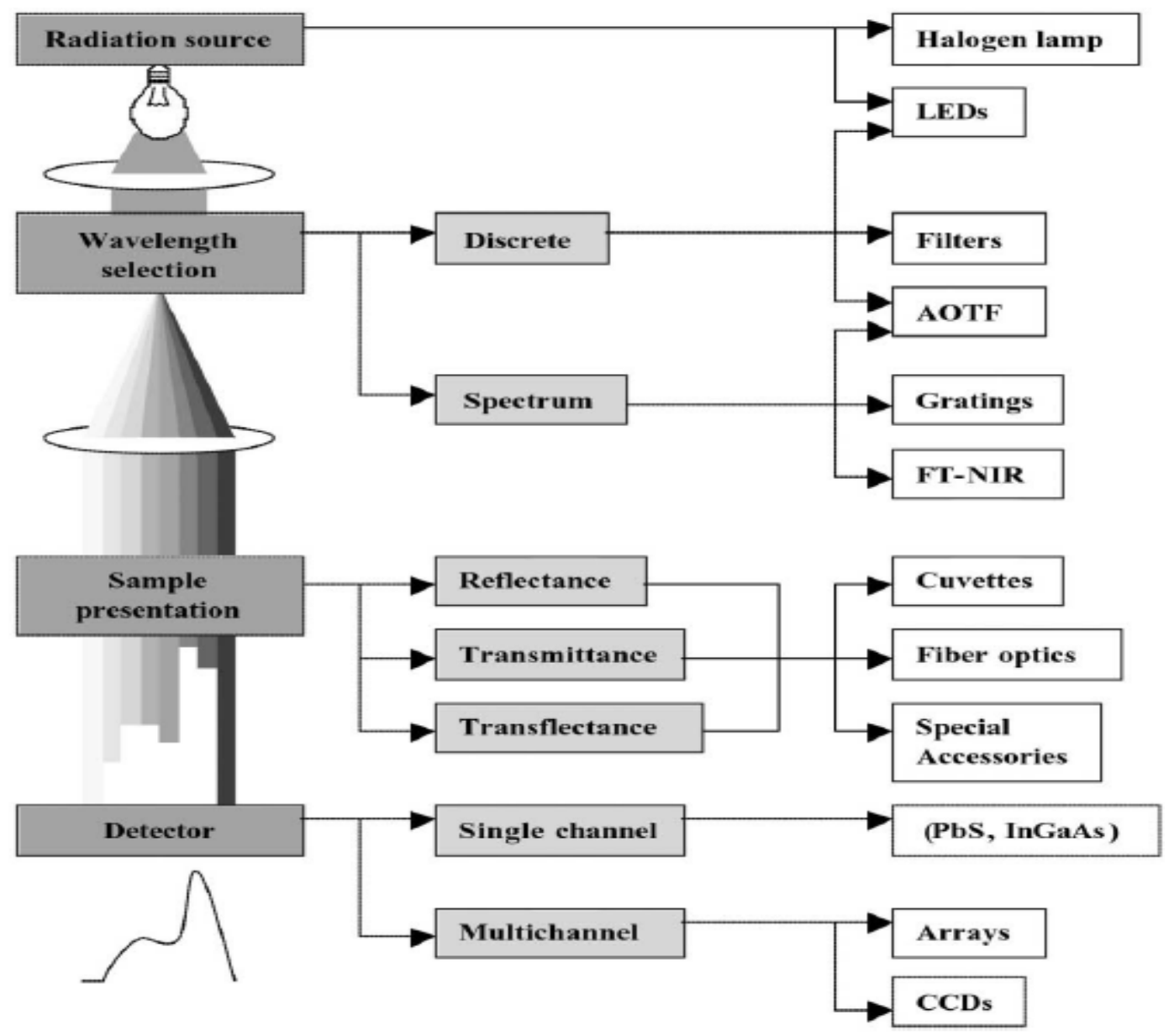

\section{Figure 2.4.2: Principal features of near-infrared spectroscopy equipment (Blanco \& Villarroya 2002)}

As mentioned earlier, to make sense of the near infra-red spectral response generated by the sample, mathematical conditioning is inevitable. A huge class of mathematical conditioning techniques that encompasses qualitative and quantitative analysis techniques is referred to as multivariate calibration. A multivariate calibration holds the relation between spectral variables, and certain sample properties for instance: analyte concentration. In addition, changes in the nearinfrared spectrum are due to changes in the properties under consideration (Hageman et al. 2005). Mathematically, any multivariate calibration aims to find the relationship as described in Equation 2.4.1. In the equation, $(\mathrm{X})$ is a regressor matrix i.e., say (m spectra with $\mathrm{n}$ wavelengths), 
(y) is a dependent variable (properties of interest, for example concentration), (b) contains regression coefficients, and (e) the residuals.

$$
y=X b+e
$$

According to Blanco \& Villarroya (2002), a number of multivariate analysis techniques can be classified according to their purpose. Figure 2.4.3 gives the most frequently used methods.

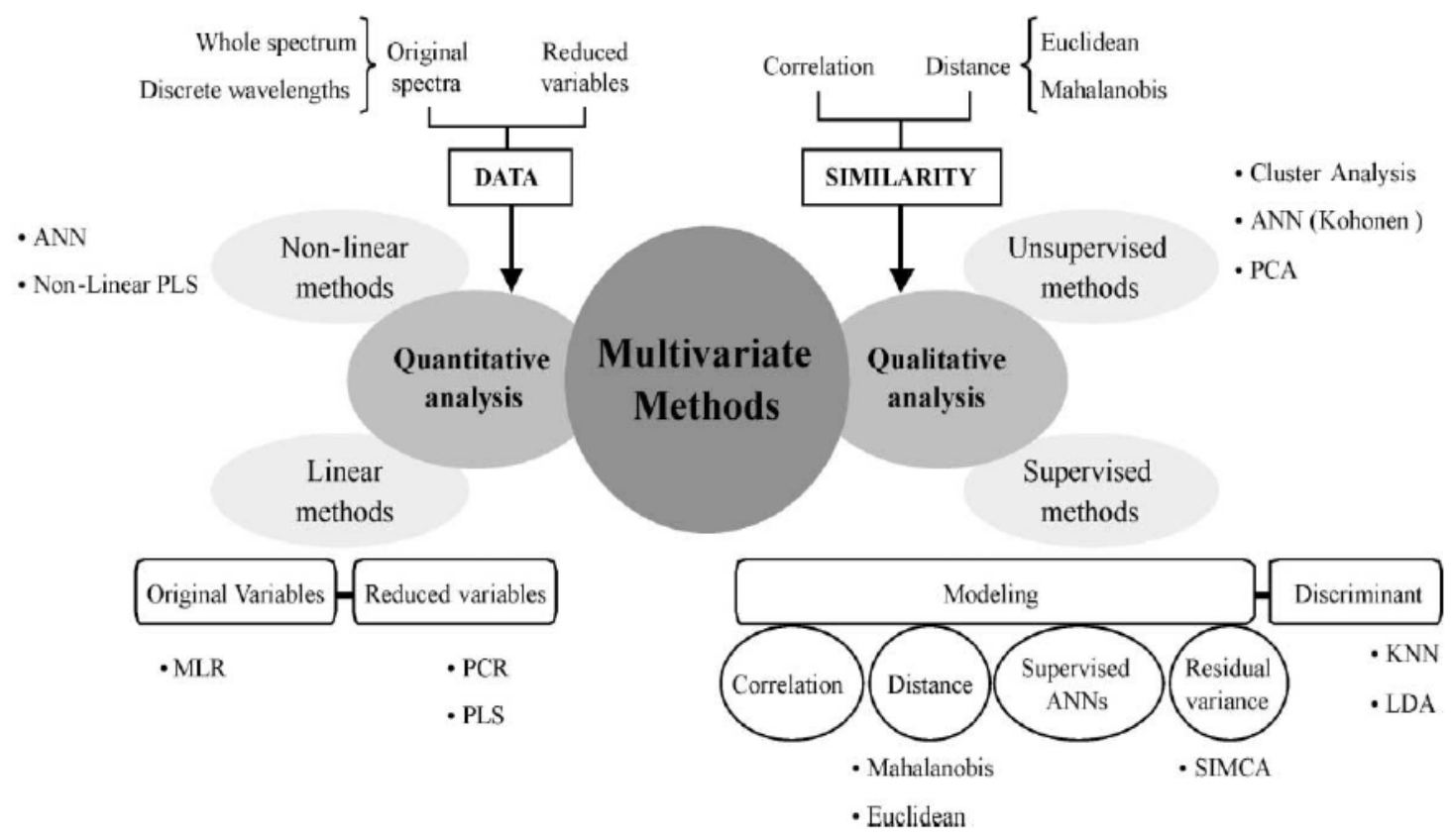

Figure 2.4.3: Qualification of major qualitative and quantitative multivariate analysis methods used in near-infra (NIR) spectroscopy (Blanco \& Villarroya 2003)

According to Vaidyanathan et al. (1999), there are three major measurement approaches associated with near-infrared spectroscopy: off-line, at-line, and on-line. Off-line measurements are measurements where the sample is taken from the process stream, and analyzed later. At-line measurements involve immediate analysis following sampling. On-line measurements can be divided into two categories ex-situ, and in-situ. Ex-situ measurements are performed by placing the device outside the perimeter, and usually involve a fiber-optic probe placed on the glass wall of the vessel or a pipe. In-situ measurements are performed by placing the sampling device inside 
the vessel where the measurements are to be taken. Figure 2.4.4 illustrates different measurement approaches.

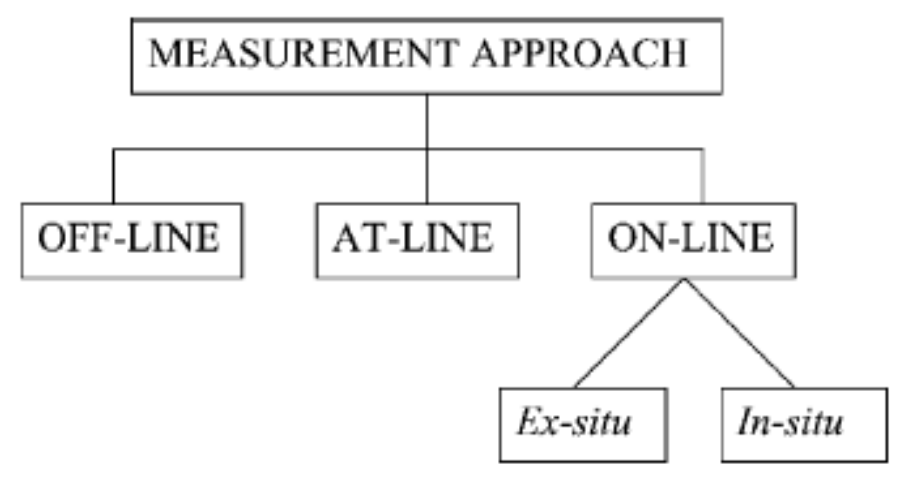

\section{Figure 2.4.4: Diagramatic representatation of different measurement approaches (Vaidyanathan et al. 1999)}

Near-infrared (NIR) spectroscopy has been rarely used to analyze slurries. Friesen (1996) used near-infrared reflectance analysis to study oil/sand slurries flowing through the pipelines of a crude oil extraction plant. A $1 \mathrm{~m}$ long fiber optic probe was coupled with a near-infrared spectrometer, and was inserted into the pipeline through a special window. The window was made out of sapphire, and hence withstood the intense abrasive nature of sand. The spectral data was collected over a period of 9 hours. It was found that the variations in the spectra were due to the variations in the type of ore and the slurry-water content.

Another documented account where near-infrared spectroscopy was used to analyze a twophase flow was presented by Switalski et al. (1999). They used the diffuse reflectance type of near-infrared spectroscopy to monitor the two-phase flow inside a production rotary dryer. Switalski et al. concluded that diffuse reflectance spectroscopy has the potential to examine both solid and liquid phases present in the flow field.

It has been mentioned earlier that the changes in the near-infrared spectrum are due to the changes in the properties of the slurry under consideration. Unfortunately, changes in the spectra 
occur for reasons other than sample compositions. When this happens, the results of multivariate calibrations lose their consistency, and will result in incorrect quantitative information (Hageman et al. 2005). These changes in the spectra can stem from change of a measuring device, or due to changes in environmental conditions (for instance different pressures or temperatures). Especially in slurry flows where the gradients in velocity, concentration, and pressure play a dominant role in spectroscopic measurement, a suitable location in a slurry flow where near-infrared spectroscopic analysis can be performed is the need of the hour. This serves as yet another incentive to study the concentration profiles of slurry exiting a contraction. Hence, a rigorous Computational Fluid Dynamic (CFD) understanding of various flow variables is what follows in the subsequent chapters. 
CHAPTER III

NUMERICAL APPROACH 


\subsection{Introduction}

In the $18^{\text {th }}$ and the $19^{\text {th }}$ century, considerable amount of work was done in order to mathematically describe the motion of fluids. Daniel Bernoulli (1700-1782) derived what is known today as Bernoulli's equation. Leonhard Euler (1707-1783) proposed Euler's equation that describes the conservation of momentum and mass for an inviscid fluid. Two other contributions made during this time would have tremendous impact in defining the field of fluid mechanics. Frenchman Claude Louis Marie Henry Navier (1785-1836) and the Irishman, George Gabriel Stokes (18191903) coupled viscous transport with the Euler equations, which resulted in the famous NavierStokes equation. These forms of differential mathematical equations postulated nearly two centuries ago form the basis of modern day computational fluid dynamics (CFD). It is debatable as to who did the earliest computational fluid dynamic calculations. There is evidence that suggests that Lewis Fry Richardson (1881-1953) in England developed the first numerical weather prediction system by dividing physical space into grid cells and used finite difference approximations of Bjerknes's "primitive differential equations"(Tokaty 1994). With the advent of mainframe and software technology, universities and national laboratories started generating computational fluid dynamic codes. Soon, commercial computational fluid dynamic codes made their presence felt in the market. Major companies around the world started accepting these codes as they promised to increase productivity and improve quality without having to invest time and money on the development of in-house computational fluid dynamic codes.

Computational fluid dynamic software (the CFD software that will be referred to from this point on is ANSYS FLUENT 12.0) is based on sets of complex non-linear mathematical expressions that define the fundamental equations of fluid flow, heat, and mass transfer. These equations are solved iteratively using complex computer algorithms embedded within the computational fluid dynamic software. Computational fluid dynamic software promises to computationally model any flow field provided the geometry of the object being modeled is 
known, the physics and chemistry are identified, appropriate and sufficient initial flow conditions and boundary conditions are prescribed. The software does not only provide reliable results in the form of numbers, but also facilitates graphical interpretation of these results by producing color plots of velocity vectors, contours of pressure, lines of constant flow field properties, and X-Y plots. In a way, the software lets the user have at his disposal, a "Virtual portable wind tunnel" where he can test prototypes subjected to a wide range of conditions without having to leave the comfort of their office space.

Computational fluid dynamics has the ability to solve problems pertaining to the opposite ends of the fluid dynamic spectrum. It can solve laminar flow problems where the Reynolds numbers are comparatively small, and on the other hand it possesses reliable recourses like Reynolds stress model, k-epsilon model, k-omega model, and Spallart-Allmaras model to account for "turbulence" which is a characteristic feature of high Reynolds number flows. These models will be dealt with greater detail in the consecutive sections of this chapter.

The ability of computational fluid dynamic software to model, not only single-phase flow, but also multi-phase flow accentuates its versatility. There is a range of widely accepted multi-phase models present in FLUENT to account for complex multi-phase flows. In the current situation, it is required for us to predict the variation of concentration profiles of a two-phase flow that exits a contraction. Generating experimental data for a multi-phase flow problem, like the one at hand, requires enormous capital, starting from the cost of the multi-phase handling equipment to the expenditure on sensitive instrumentation, and data acquisition. Thus, the use of industry accepted multi-phase and turbulence models to analyze a multi-phase flow is definitely a fruitful option. All this can be accomplished at a fraction of the cost and the time of what would be needed in case of an experimental endeavor.

Any computational fluid dynamic problem is solved in three steps, namely: pre-processing, solving, and post-processing. Pre-processing is where the geometry is created and discretized into 
smaller volumes. These volumes are also called cells. The region of interest where numerical data has to be computed is also defined in the pre-processing phase. The solving process is where the created geometry is uploaded into solving software, and the boundary conditions are specifically defined. Later, the numerical representations of the governing equations are solved iteratively at the nodes of the previously created volumes. The results are numerically interpreted in the postprocessing stage. The aforementioned stages will become clear as the reader goes through the consecutive sections of this chapter.

There is a plethora of commercial computational fluid dynamic software available in the market today. As ICEM CFD, FLUENT (both belong to ANSYS, Inc) possess an exceedingly easy-to-use graphical-user-interface (GUI), and at the same time entail an overwhelming reputation in the industry, they were used as the primary software(s) to perform pre-processing, solving, and post-processing operations in this study.

\subsection{Governing equations}

The three basic equations that play a crucial role in defining a flow field are the conservation of mass, momentum, and energy. These equations will be presented momentarily. The equation of conservation of mass which is also known as the continuity equation, and the equation of conservation of momentum which is referred to as the Navier-Stokes equation in the case of Newtonian fluids will be presented first. The three dimensional continuity equation is represented by Eq. 3.2.1 where $\mathrm{X}, \mathrm{Y}$, and $\mathrm{Z}$ are three co-ordinate axes.

$$
\frac{\partial u}{\partial x}+\frac{\partial v}{\partial y}+\frac{\partial w}{\partial z}=0
$$

Technically speaking, Eq. 3.2.1 is the continuity equation for an incompressible flow where $\mathrm{u}, \mathrm{v}$, and $\mathrm{w}$ represent the velocity components in $\mathrm{X}, \mathrm{Y}$, and $\mathrm{Z}$ directions respectively. 
Before discussing the equation of conservation of momentum (Navier-Stokes equation), it's best to present Newton's second law of motion. This is justified by the fact that Newton's second law is theoretically simpler to understand. It is presented in Eq. 3.2.2.

$$
m \frac{d v}{d t}=\sum F
$$

Newton's law states that the rate of change of momentum of a body is directly proportional to the summation of all the forces acting on it. This equation cannot be applied directly to fluids as they are not rigid. Thus, one makes use of the Navier-Stokes equation. It is presented in Eq. 3.2.3.

$$
\rho\left(\frac{\partial v}{\partial t}+v \cdot \nabla v\right)=-\nabla p+\mu \nabla^{2} v+f
$$

Similar to Eq. 3.2.2, the left hand side of the Navier-Stokes equation represents the acceleration of a fluid element while the right hand side presents the forces acting on it. Figure 3.2.1 illustrates what the individual terms in the Navier-Stokes equation stand for.

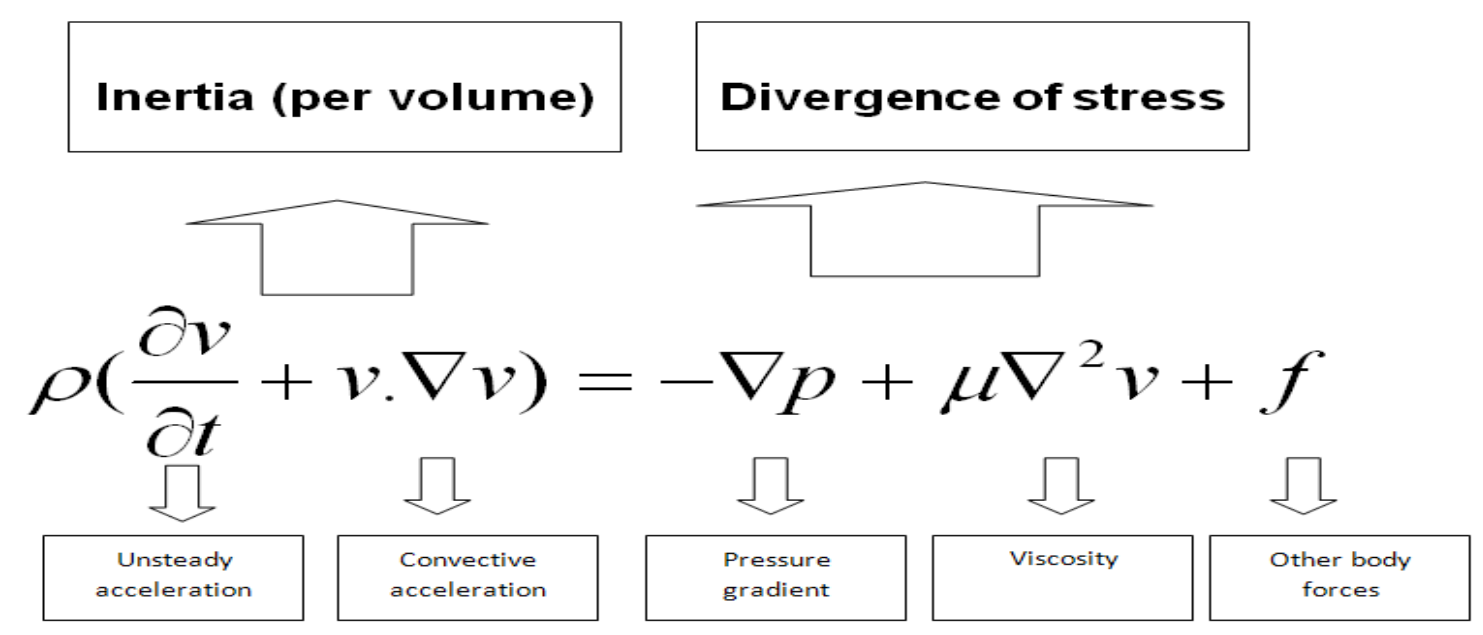

Figure 3.2.1: Illustration of all the terms in the Navier-Stokes equation

The energy equation is presented in Equation 3.2.4. 


$$
\frac{d Q}{d t}-\frac{d W}{d t}=\frac{d E}{d t}
$$

Using Navier-Stoke's equation, in the form described previously, it is not computationally feasible to account for turbulence which is characteristic of high Reynolds number pipe flows. However, Direct Numerical Simulation (DNS) lets one describe a fluid flow by resolving a whole range of spatial and temporal scales of turbulence. This requires tremendous computational resources. Another technique that requires comparatively less computational resources is Large Eddy Simulation (LES). As the term indicates, this technique resolves only the large, high energy eddies that govern the turbulent flow field. The smaller, dissipative eddies are mathematically modeled. The third class of turbulence modeling techniques is the Reynolds Averaged Navier Stokes (RANS). All the commercial computational fluid dynamic codes use RANS models. FLUENT not only incorporates RANS models but also has the ability to perform large eddy simulations. Since the turbulent flow field is going to be simulated using a model based on RANS scheme, it is worthwhile to describe this concept first.

Applying time averaging to the basic equation of motion produces what is famously known as the Reynolds equation (White 2006). Its simplistic approach has often resulted in the underestimation of its promising capabilities. But the Reynolds equations have stood the test of time, and are extensively used as the basis for solving a wide array of turbulent flow problems.

The process of applying Reynolds averaging to the Navier-Stokes equation (equation of motion) is also called Reynolds decomposition for reasons that will become evident momentarily. The underlying principle is to treat every quantity in the transport equation as a sum of a mean quantity and its corresponding fluctuating counterpart. The following equation set aids the understanding of this treatment. The terms represented by a bar $(\bar{u})$ on top are the mean terms and the ones represented by a dash $\left(u^{\prime}\right)$ are the fluctuating terms.

$$
u=\bar{u}+u^{\prime}
$$




$$
\begin{gathered}
v=\bar{v}+v^{\prime} \\
w=\bar{w}+w^{\prime} \\
p=\bar{p}+p^{\prime} \\
T=\bar{T}+T^{\prime}
\end{gathered}
$$

All the terms in the Navier-Stokes equation are decomposed and eventually subjected to time averaging. Thus, when the rules of averaging are applied, and the equation is further simplified, the resultant form is drastically different from what one starts with. The average of a fluctuating term multiplied to a mean term vanishes, while the average of two fluctuating terms remains. The average of two fluctuating terms is the cause of all evil in the Reynolds Averaged Navier-Stokes equation, and has to be mathematically modeled for its effect to be represented. The Reynolds Averaged Navier-Stokes equation is presented below.

$$
\begin{gathered}
\rho \frac{D \bar{V}}{D t}=\rho g-\nabla \bar{p}+\nabla \cdot \tau_{i j} \\
\tau_{i j}=\mu\left(\frac{\partial \overline{u_{i}}}{\partial x_{j}}+\frac{\partial \overline{u_{j}}}{\partial x_{i}}\right)-\rho \overline{u_{i}^{\prime} u_{j}^{\prime}}
\end{gathered}
$$

The mean momentum equation (Eq. 3.2.6 (a)) becomes complicated due to the appearance of a new term as a result of decomposition followed by time averaging. This new term $\left(-\rho \overline{u_{i}^{\prime} u_{j}^{\prime}}\right)$, is called the turbulent stress tensor, and it can never be neglected in any turbulent flow. Mathematically, turbulent stress terms behave as if the total stress on the system were composed of Newtonian viscous stresses and an additional turbulent stress tensor. Almost all numerical complications stem from these terms, predominantly because their analytic form is not known. Thus, the time averaging process has introduced nine new variables (the tensor components) that can be fathomed only through (unavailable) knowledge of detailed turbulent structure (White 
2006). The effect of these turbulent stresses on the flow field is simulated by either modeling them algebraically or through additional partial differential equations. These two approaches are dealt with in greater detail in Section. 3.3 of this chapter.

Turbulence has been painted in various colors over the years. Lamb (1916) quotes: "turbulence is the chief outstanding difficulty of our subject". Bradshaw (1994) states: "turbulence is an invention of the Devil on the seventh day of creation". More technically so, Kline et al. (1967), while studying turbulent boundary layers, quotes: "Turbulence is neither completely ordered nor completely random, but somewhere in between because of the existence of coherent structures". This leads one to believe that the effect of turbulence on particles suspended in the flow cannot be purely dispersive and/or random in nature. Thus, a turbulence model that would predict the exact behavior of particles suspended in the flow must be selected.

\subsection{Turbulence models and their selection}

The process of choosing a turbulence model depends primarily on the amount of computational resource available, type of flow (internal, external), the physics of the flow, and finally the level of accuracy desired. Direct numerical simulation (DNS) and large eddy simulation (LES) produce supreme degrees of resolution. Unfortunately, they also require enormous amounts of computational power. However, Reynolds averaged Navier Stokes (RANS) are turbulence models that require paltry (in comparison to DNS and LES) computational resources yet are capable of producing commendable degrees of accuracy, provided that the problem is posed correctly. Hence, Reynolds Averaged Navier Stokes will be the subject of discussion in the present section. The following paragraphs briefly, yet effectively, summarize the different models that belong to the aforementioned regime.

The realm of RANS can be split into two groups. There are models that are based on what is known as the Boussinesq hypothesis, and ones that are referred to as Reynolds stress models. The Boussinesq hypothesis involves using algebraic equations for Reynolds stresses. The Reynolds 
stress models solve the transport equations to discern the generated Reynolds stresses. The Reynolds stress models are mathematically complex as they solve additional 7 partial differential equations.

Most of the turbulence models that FLUENT offers are based on the Boussinesq hypothesis. These models are either one equation models or two equation models. One equation models are predominantly used to solve external flows (these flows can have walls too). The SpalartAllmaras model is known to produce good results for external flows. Since the flow in question is a pipe flow, models that hold the capability to resolve internal flows will be discussed. The most widely used two equation model is the k-epsilon model. It solves two additional governing equations, turbulent kinetic energy $(\mathrm{k})$, and viscous dissipation $(\varepsilon)$ in order to compute the contributions of turbulence to the mean flow. This model captures turbulent activity away from the wall, but can be equipped with wall functions that make it work near the wall also. Before presenting the k-epsilon model, it is worthwhile to understand the concept of Boussinesq eddy viscosity. Boussinesq postulated that the momentum transfer caused by turbulent eddies can be modeled with an eddy viscosity. The underlying principle is that the Reynolds stress tensor $\left(\tau_{i j}\right)$

is proportional to the mean strain rate tensor $\left(S_{i j}{ }^{*}\right)$, and can be written according to equation 3.3.1.

$$
\tau_{i j}=2 \mu_{t} S_{i j}^{*}-\frac{2}{3} \rho k \delta_{i j}
$$

The following equation set defines the eddy viscosity and presents equations for the k-epsilon turbulence model (Pope 2006).

$$
\begin{gathered}
\mu_{t}=\rho C_{\mu} \frac{k^{2}}{\varepsilon} \\
\frac{\partial}{\partial t}(\rho k)+\frac{\partial}{\partial x_{i}}\left(\rho k \overline{u_{i}}\right)=\frac{\partial}{\partial x_{j}}\left[\left(\mu+\frac{\mu_{t}}{\sigma_{k}}\right) \frac{\partial k}{\partial x_{j}}\right]+P_{k}-\rho \varepsilon+S_{k}
\end{gathered}
$$




$$
\begin{gathered}
\frac{\partial}{\partial t}(\rho \varepsilon)+\frac{\partial}{\partial x_{i}}\left(\rho \varepsilon \overline{u_{i}}\right)=\frac{\partial}{\partial x_{j}}\left[\left(\mu+\frac{\mu_{t}}{\sigma_{\varepsilon}}\right) \frac{\partial \varepsilon}{\partial x_{j}}\right]+C_{1 \varepsilon} \frac{\varepsilon}{k}\left(P_{k}+C_{3 \varepsilon} P_{b}\right)-C_{2 \varepsilon} \rho \frac{\varepsilon^{2}}{k}+S_{\varepsilon} \\
P_{k}=-\rho \overline{u_{i}^{\prime} u_{j}^{\prime}} \frac{\partial u_{j}}{\partial x_{i}} \\
S=\sqrt{2 S_{i j} S_{i j}}
\end{gathered}
$$

Turbulent flows are significantly affected when they are bounded by walls. Very close to the wall, viscous damping reduces the tangential velocity fluctuations, while kinematic blocking reduces normal fluctuations. Further, near wall modeling dearly impacts the credibility of the numerical solution. The near-wall region generates large gradients, and scalar transports occur in a very feverish manner. Therefore, accurate representation of the flow in the near-wall region determines successful prediction of wall bounded turbulent flows. The k-epsilon model is generally suitable for turbulent core flows. On the other hand the k-omega model is suitable for near-wall flows, provided that the region close to the wall has a very fine mesh. Solving turbulent flows where the resolution close to the wall is fine demands large computational power. Thus, the computational fluid dynamic software (FLUENT) is equipped with what are known as "wall functions". These functions are semi-empirical formulas used to bridge the viscosity-affected region between the wall and the fully-turbulent region. The use of wall functions obviates the need to modify the turbulence models to account for the presence of the wall. The so called wall functions can be understood by observing Fig 3.3.1. 


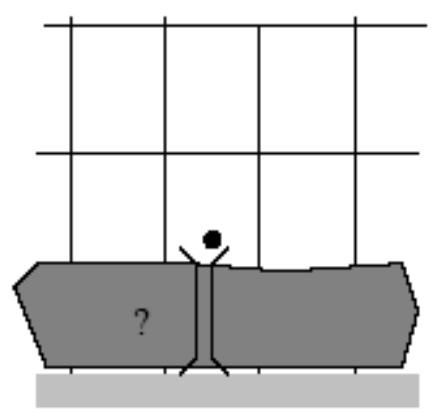

\section{Wall Function Approach}

The viscosity-affected region is not resolved, instead is bridged by the wall functions.

High-Re turbulence models can be used.

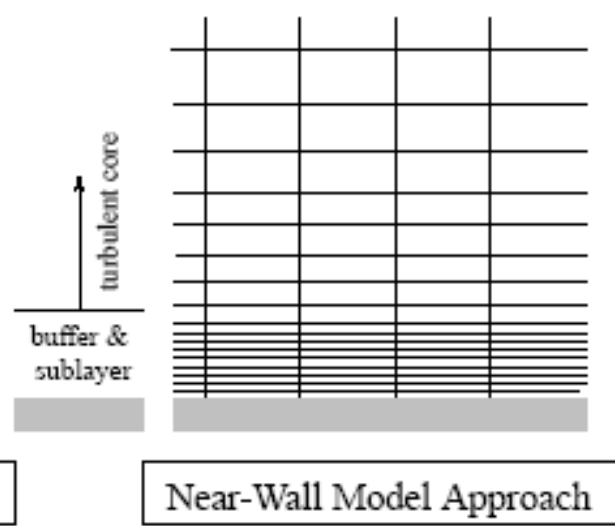

- The near-wall region is resolved all the way down to the wall.

- The turbulence models ought to be valid throughout the near-wall region.

Figure 3.3.1: Near-wall treatments (Hirsch 2007)

Since the k-epsilon turbulence model provides a wide array of wall functions that are apt for industrial flows, the decision of choosing this model to solve a slurry flow through a contraction seems to be logical. Now that a suitable turbulence model is selected, the next step is to select a suitable multi-phase model that would account for particle-fluid and particle-particle interaction.

\subsection{Selection of multi-phase flow model}

A multi-phase flow has more than one phase present inside the flow field. The present work deals with turbulent two-phase flows where the primary phase is liquid and the secondary or the disperse phase is the solid particles. There are two approaches that are most prevalently used to model multi-phase flows: the Euler-Lagrange approach and the Euler-Euler approach.

In the Euler-Euler approach, the different phases are treated mathematically as interpenetrating media. Since the volume of a phase cannot be occupied by the other phases, the concept of phase volume fraction is introduced. These volume fractions are assumed to be continuous functions of space and time and their sum is equal to one. Conservation equations for each phase are derived to obtain a set of equations, which have similar structure for all phases. These equations are 
closed by providing constitutive relations that are obtained from empirical information. While on the other hand, in the Euler-Lagrange approach, the fluid phase is treated as a continuum by solving the time-averaged Navier-Stokes equations, while the dispersed phase is solved by tracking a large number of particles, bubbles, or droplets through the calculated flow field. The dispersed phase can exchange momentum, mass, and energy with the fluid phase. A fundamental assumption made in this model is that the dispersed phase occupies a low volume fraction. This makes the model appropriate for the modeling of spray dryers, coal, and liquid fuel combustion, but inappropriate for the modeling of liquid-liquid mixtures, fluidized beds, or any application where the volume fraction of the dispersed phase is not negligible.

Since the slurry that will be modeled in this work has a considerable amount of secondary phase (disperse phase) volume fraction, using Euler-Lagrange approach to model it is out of the question. There are three different models in FLUENT that are based on the Euler-Euler approach namely: the volume of fluid (VOF) model, the mixture model, and the Eulerian model. They will be described briefly in the paragraphs that follow.

The VOF model is a surface-tracking technique applied to a fixed Eulerian mesh. It is designed for two or more immiscible fluids where the position of the interface between the fluids is of interest. In the VOF model, a single set of momentum equations is shared by the fluids, and the volume fraction of each of the fluids in each computational cell is tracked throughout the domain. Further, this model can handle stratified flows, free-surface flows, filling, sloshing, the motion of large bubbles in a liquid, the motion of liquid after a dam break, the prediction of jet breakup (surface tension), and the steady or transient tracking of any liquid-gas interface.

The mixture model is designed for two or more phases (fluid or particulate). As in the Eulerian model, the phases are treated as interpenetrating media. The mixture model solves for the mixture momentum equation and prescribes relative velocities to describe the dispersed phases. In addition, this model can handle particle-laden flows with low loading, bubbly flows, sedimentation, and cyclone separators. 
The Eulerian model is the most complex of the multiphase models in FLUENT. It solves a set of $\mathrm{n}$ momentum and continuity equations for each phase. Coupling is achieved through the pressure and interphase exchange coefficients. The manner in which this coupling is handled depends upon the type of phases involved; granular (fluid-solid) flows are handled differently than non-granular (fluid-fluid) flows. For granular flows, the properties are obtained from application of kinetic theory. Momentum exchange between the phases is also dependent upon the type of mixture being modeled. Further, this model can handle bubble columns, risers, particle suspension, and fluidized beds.

From the above discussion it becomes quite evident that the volume of fluid (VOF) model cannot be applicable for the problem at hand. Thus, one is left to choose between the mixture model and the Eulerian model. Before one goes ahead and chooses between the two models, it's a good idea to talk about the fluid-particle and particle-particle interactions. It is best to select a model that accounts for these interactions without demanding huge computational effort. Furthermore, since this work tries to predict the concentration profiles of particle-laden slurry exiting a geometric contraction, the interaction between the two phases becomes a primary concern. The Stokes number is one of the best parameters that can quantify the interaction between the phases in question, and can help one select the most appropriate multi-phase model. Stokes number gives the relation between the particle response time $\left(\tau_{d}\right)$ and the system response time $\left(t_{s}\right)$ (Crowe et al. 1998):

$$
\begin{gathered}
S t=\frac{\tau_{d}}{t_{s}} \\
\tau_{d}=\frac{\rho_{d} d_{p}^{2}}{18 \mu} \\
t_{s}=\frac{L_{s}}{V_{s}}
\end{gathered}
$$


In the above equation set, $t_{s}$ is based on characteristic length $L_{s}$ and characteristic velocity $V_{s}$. For the system that is going to be investigated in this work, the maximum values of all the quantities that appear in equation set 3.4.1 are presented in the table below.

\begin{tabular}{|c|c|c|c|c|}
\hline$\rho_{d}\left(\mathrm{Kg} / \mathrm{m}^{3}\right)$ & $d_{p}(\mu \mathrm{m})$ & $\mu(P a-s)$ & $L_{s}(\mathrm{~m})$ & $V_{s}(\mathrm{~m} / \mathrm{s})$ \\
\hline 1480 & 150 & $6.2 \times 10^{-4}$ & 0.045 & 16 \\
\hline
\end{tabular}

\section{Table 3.4.1: The maximum values of all the quantities required to calculate the Stokes number}

The characteristic length $\left(L_{s}\right)$ is the axial length scale of the nozzle section of the geometric contraction. And the characteristic velocity $\left(V_{s}\right)$ is the maximum velocity that is registered in this section. Thus when we substitute all the values mentioned in Table 3.4.1 into equation set 3.4.1, we find that the Stokes number (St) satisfies St 1. This implies that the disperse phase follows the continuous phase closely. Thus in a situation like this, one is free to choose between the mixture model and the Eulerian multi-phase model. The mixture model is chosen as it is considered to be the most inexpensive, in a computational sense, of the two multi-phase models.

\subsubsection{Modeling with mixture model}

The mixture model solves only one set of continuity and momentum equations for the whole mixture, as opposed to the Eulerian model that solves ' $n$ ' momentum and continuity equations for each phase. This is the primary reason for choosing the mixture model over the Eulerian model. Apart from solving one set of continuity, momentum, and energy equations for the mixture, the mixture model also solves the volume fraction equation for the secondary phase. It also accounts for the relative velocity between the two phases if they are moving at different velocities. The equations that the mixture model employs are described in the following paragraphs.

As mentioned previously, the continuity equation is solved by the mixture model. It is 
presented in Eq. 3.4.1.1.

$$
\begin{gathered}
\frac{\partial\left(\rho_{m}\right)}{\partial t}+\nabla \cdot\left(\rho_{m} \overline{v_{m}}\right)=0 \\
v_{m}=\frac{\sum_{k=1}^{n} \alpha_{k} \rho_{k} \overline{v_{k}}}{\rho_{m}} \\
\rho_{m}=\sum_{k=1}^{n} \alpha_{k} \rho_{k}
\end{gathered}
$$

Where $\overline{v_{k}}$ and $\rho_{m}$ represent mass averaged velocity mixture density, and $\alpha_{k}$ is the volume fraction of $\mathrm{k}^{\text {th }}$ phase. Further, the momentum equation solved by the mixture model is given by:

$$
\begin{gathered}
\frac{\partial\left(\rho_{m} \overline{v_{m}}\right)}{\partial t}+\nabla \cdot\left(\rho_{m}{\overline{v_{m} \cdot v_{m}}}^{\prime}\right)=-\nabla p+\nabla \cdot\left[\mu_{m}\left(\nabla \overline{v_{m}}+\nabla \overline{v_{m}^{T}}\right)\right]+\rho_{m} \bar{g}+\bar{F}+\nabla \cdot\left[\sum_{k=1}^{n} \alpha_{k} \rho_{k} \overline{v_{d r, k}} \overline{v_{d r}}\right. \\
\mu_{m}=\sum_{k=1}^{n} \alpha_{k} \mu_{k} \\
\overline{v_{d, k}}=\overline{v_{k}}-\overline{v_{m}}
\end{gathered}
$$

Where n, $\bar{F}$, and $\mu_{m}$ are number of phases, total body forces, and viscosity of the mixture respectively, and $\overline{v_{d r, k}}$ is the drift velocity. The energy equation is given in Eq. 3.4.1.3.

$$
\begin{gathered}
\frac{\partial}{\partial t} \sum_{k=1}^{n}\left(\alpha_{k} \rho_{k} E_{k}\right)+\nabla \cdot \sum_{k=1}^{n}\left(\alpha_{k} \overline{v_{k}}\left(\rho_{k} E_{k}+p\right)\right)=\nabla .\left(k_{e f f} \nabla T\right)+S_{E} \\
k_{e f f}=\sum\left(\alpha_{k}\left(k_{k}+k_{t}\right)\right)
\end{gathered}
$$

The first and second terms in the energy equation stand for conduction heat transfer and 
miscellaneous volumetric heat sources. The subscript $\mathrm{t}$ in the conductivity term $k_{t}$ stands for turbulence, and its value varies with turbulence model selected. The volume fraction equation that the mixture model solves is derived from the continuity equation. It is presented in Eq. 3.4.1.4 (FLUENT Inc 2009).

$$
\frac{\partial}{\partial t}\left(\alpha_{p} \rho_{p}\right)+\nabla \cdot\left(\alpha_{p} \rho_{p} \overline{v_{m}}\right)=-\nabla \cdot\left(\alpha_{p} \rho_{p} \overline{v_{d r, p}}\right)+\sum_{q=1}^{n}\left(m_{q p}-m_{p q}\right)
$$

Finally, the relative or the slip velocity between the two phases that the mixture model accounts for is described by the following set of equations.

$$
\begin{gathered}
\overline{v_{p q}}=\overline{v_{p}}-\overline{v_{q}} \\
\overline{v_{d r, p}}=\overline{v_{p q}}-\sum_{k=1}^{n} c_{k} \overline{v_{q k}} \\
c_{k}=\frac{\alpha_{k} \rho_{k}}{\rho_{m}} \\
v_{p q}=\frac{\left(\rho_{p}-\rho_{m}\right) d_{p}^{2}}{18 \mu_{q} f_{d r a g}} \vec{a}-\frac{v_{m}}{\alpha_{p} \sigma_{D}} \nabla \alpha_{q}
\end{gathered}
$$

Where,

$c_{k} \quad=\quad$ mass fraction of any phase

$\vec{a} \quad=\quad$ acceleration of disperse phase

$f_{\text {drag }}=$ drag function

$\sigma_{D} \quad=\quad$ Prandlt dispersion coefficient 
The only aspect of the multi-phase flow that has not been accounted for is the turbulent structures that the primary phase generates. The behavior of the particles in the presence of turbulent structures is described by the k-epsilon model that was chosen earlier. Turbulent kinetic energy (k) and dissipation (epsilon) when coupled with the mixture model are transformed to the following equations (FLUENT Inc 2009).

$$
\begin{aligned}
& \frac{\partial}{\partial t}\left(\rho_{m} k\right)+\nabla \cdot\left(\rho_{m} \overline{v_{m}} k\right)=\nabla \cdot\left(\frac{\mu_{t, m}}{\sigma_{k}} \nabla k\right)+G_{k, m}-\rho_{m} \varepsilon \\
& \frac{\partial}{\partial t}\left(\rho_{m} \varepsilon\right)+\nabla \cdot\left(\rho_{m} \overline{v_{m}} \varepsilon\right)=\nabla \cdot\left(\frac{\mu_{t, m}}{\sigma_{\varepsilon}} \nabla \varepsilon\right)+\frac{\varepsilon}{k}\left(C_{1 \varepsilon} G_{k, m}-C_{2 \varepsilon} \rho_{m} \varepsilon\right)
\end{aligned}
$$

Where,

$$
\begin{gathered}
\rho_{m}=\sum_{i=1}^{n} \alpha_{i} \rho_{i} \\
\overline{v_{m}}=\frac{\sum_{i=1}^{n} \alpha_{i} \rho_{i} \overline{v_{i}}}{\sum_{i=1}^{n} \alpha_{i} \rho_{i}}
\end{gathered}
$$

And turbulent viscosity is given by Eq. 3.4.1.10.

$$
\mu_{t, m}=\rho_{m} C_{\mu} \frac{k^{2}}{\varepsilon}
$$

Now that all the details of the various models that are going to be incorporated have been specified, the next stage of the process would be to generate the geometry. After generating the geometry, a suitable discretization scheme will be applied, and the process of solving the flow field will follow. The details of the problem, pre-processing, solving, and post-processing will be discussed in Sections 3.5, 3.6, 3.7, and 3.8 respectively. 


\subsection{Problem details}

This section presents the details of the geometry that is considered for performing computational fluid dynamic analysis of the two-phase flow. In addition, the properties of the primary and secondary phases of the slurry are also discussed.

Since this work primarily aims to study the concentration profiles of a slurry travelling through a symmetric contraction, it is necessary to define the dimensions of this structure. An ASME flow nozzle with a $\beta$ value of 0.5 is chosen to serve the purpose of a symmetric contraction. Beta $(\beta)$ is the ratio of the outlet diameter to the inlet diameter of the nozzle. Its dimensions are illustrated in Fig 3.5.1.

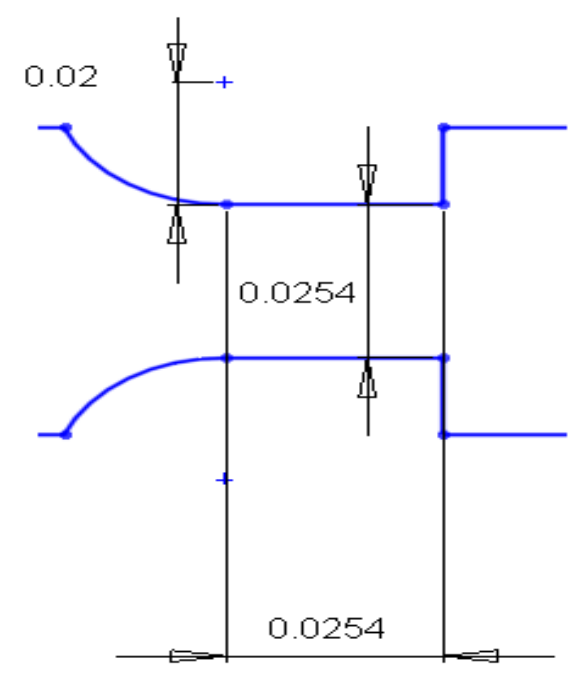

Figure 3.5.1: Schematic of an ASME flow nozzle $(\beta=0.5)$

This contraction is incorporated in a pipe section at a suitable location that will be decided on the basis of the following discussion.

The diameter (D) of the pipe in question is selected $0.0508 \mathrm{~m}$ ( 2 inches). All the simulations are performed at an average primary phase velocity of $2 \mathrm{~m} / \mathrm{s}$ or $4 \mathrm{~m} / \mathrm{s}$. Thus, for a slurry that is 
flowing in these conditions, it requires at least 20 pipe diameters to become fully-developed. To be absolutely certain that fully-developed conditions have been established at the time that the slurry reaches the nozzle, the nozzle is placed at a distance of 35 pipe diameters from the inlet. At the end of the nozzle, an extra 20 diameters of pipe is added. In summary, we are dealing with a pipe that is $2.8 \mathrm{~m}$ (111.75 inches) long. This produces an 1/D ratio of approximately 55 . Figure 3.5.2 presents a schematic of the geometry that is described above.

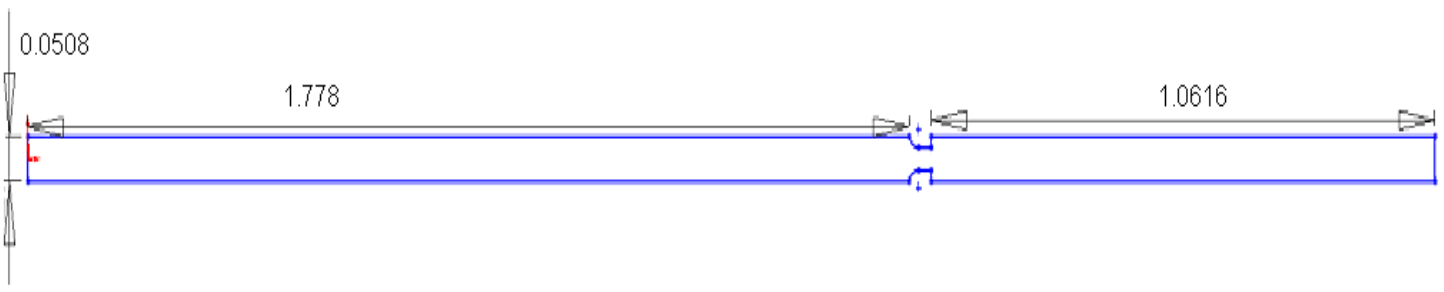

Figure 3.5.2: Schematic of the pipe section in corporate with an ASME flow nozzle

Now that the geometric details have been specified the next step is to deal with the properties of the two-phase flow in question. The two-phase flow that is going to be simulated has xylene as the primary phase and 2-Amino-4, 6-dimethylpyrimidine (ADP) as the secondary or the disperse phase. The properties of these two phases are presented Table. 3.5.1.

\begin{tabular}{|c|c|c|}
\cline { 2 - 3 } \multicolumn{1}{c|}{} & Primary phase & Secondary phase \\
\hline Material & Xylene & ADP \\
\hline Phase type & Liquid & Solid \\
\hline Density $\left(\mathrm{Kg} / \mathrm{m}^{3}\right)$ & 870 & 1480 \\
\hline Viscosity $(\mathrm{Pa}-\mathrm{s})$ & $6.2 \times 10^{-4}$ & N/A \\
\hline Particle size $(\mu \mathrm{m})$ & N/A & $38-150$ \\
\hline
\end{tabular}

Table 3.5.1: Information with regards to the primary and secondary phases of the slurry 


\subsection{Pre-Processing}

The primary step in simulating any fluid dynamic problem computationally, involves preprocessing. As mentioned previously, pre-processing involves the building of geometry, discretizing the volume, followed by applying the boundary conditions at the prescribed boundaries. All these steps and the thought process that went into choosing from a wide variety of available options will be presented in this section.

ICEM CFD was used as the primary agent to perform pre-processing. All the co-ordinates of the geometry that were described in Section 3.5 were entered into the software. A volume was generated by revolving an edge around the axis of the geometry. The isometric view of the mentioned volume is presented in Fig 3.6.1.

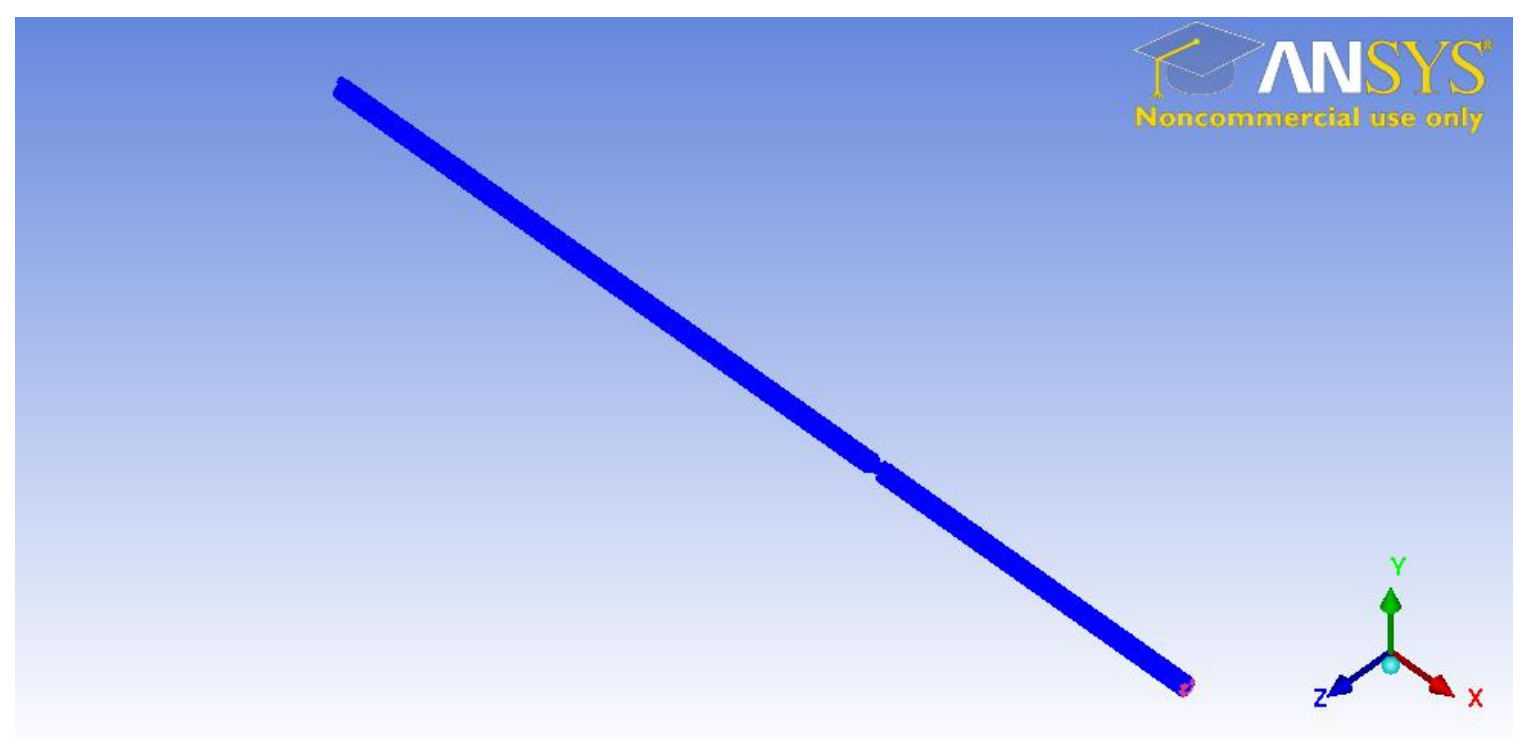

Figure 3.6.1: An isometric view of the pipe-nozzle assembly

Applying a mesh to the created volume is what follows. This step is of paramount importance in the whole pre-processing procedure, if not the entire numerical treatment. Selection of a suitable mesh, and also the right number of cells is vital in deciding the accuracy of the obtained results. The meshing scheme is decided after describing the different types of meshes available at 
our disposal. The meshes are broadly classified into structured and unstructured meshes. Structured meshes acquire their name from the fact that the mesh is laid out in a regular repeating pattern called a block. These types of grids utilize quadrilateral elements in 2D and hexahedral elements in $3 \mathrm{D}$ in a computationally rectangular array. The primary drawback of structured meshes is that they are suitable only for simple geometries. Creating an efficient structured mesh for complex geometries demands huge computational effort. In contrast, unstructured grid methods utilize a random collection of elements to fill the region. As the arrangements of elements have no discernible pattern, the mesh is called unstructured. These types of grids typically utilize triangles in 2D and tetrahedral in 3D. Unstructured meshes complement complex geometries well, and their generation requires comparatively lower computational resources (Hirsch 2007).

Owing to the fact that the nozzle has a combination of curved and sharp boundaries, it was decided that an unstructured meshing scheme would serve the purpose of resolving the turbulent pipe flow. However, it is worth mentioning that a Cartesian (structured mesh) meshing scheme was tried, but the solution failed to converge. The reasons for this occurrence are unknown. Thus, a tetrahedral meshing scheme was selected. The details of the meshing scheme are presented in Table 3.6.1. It should be noted here that the tetrahedral grid was adapted, after generating an initial steady state solution, until the condition: $30<y^{+}<60$ was satisfied. If this condition is not satisfied, one cannot be sure of achieving accurate computational results using the k-epsilon model with standard wall functions of FLUENT. However, it is worth noting that kepsilon model with standard wall functions is efficient enough to produce accurate solutions even when $\mathrm{y}^{+}$is greater than 60 but less than 300 . The concept of $\mathrm{y}^{+}$can be understood after going through the following paragraphs.

The near-wall region is subdivided into three layers. The inner most layer is called the viscous sub layer. It is in this layer that the viscous forces are dominant, and molecular viscosity governs momentum and heat transfer. The outer most layer is called the fully-turbulent layer where the 
turbulence plays a dominant role. There is also a layer present in the middle where both the viscous and turbulent forces play a crucial role (Wilcox 1993). Figure 3.6 .2 illustrates the subdivisions of the near-wall region.

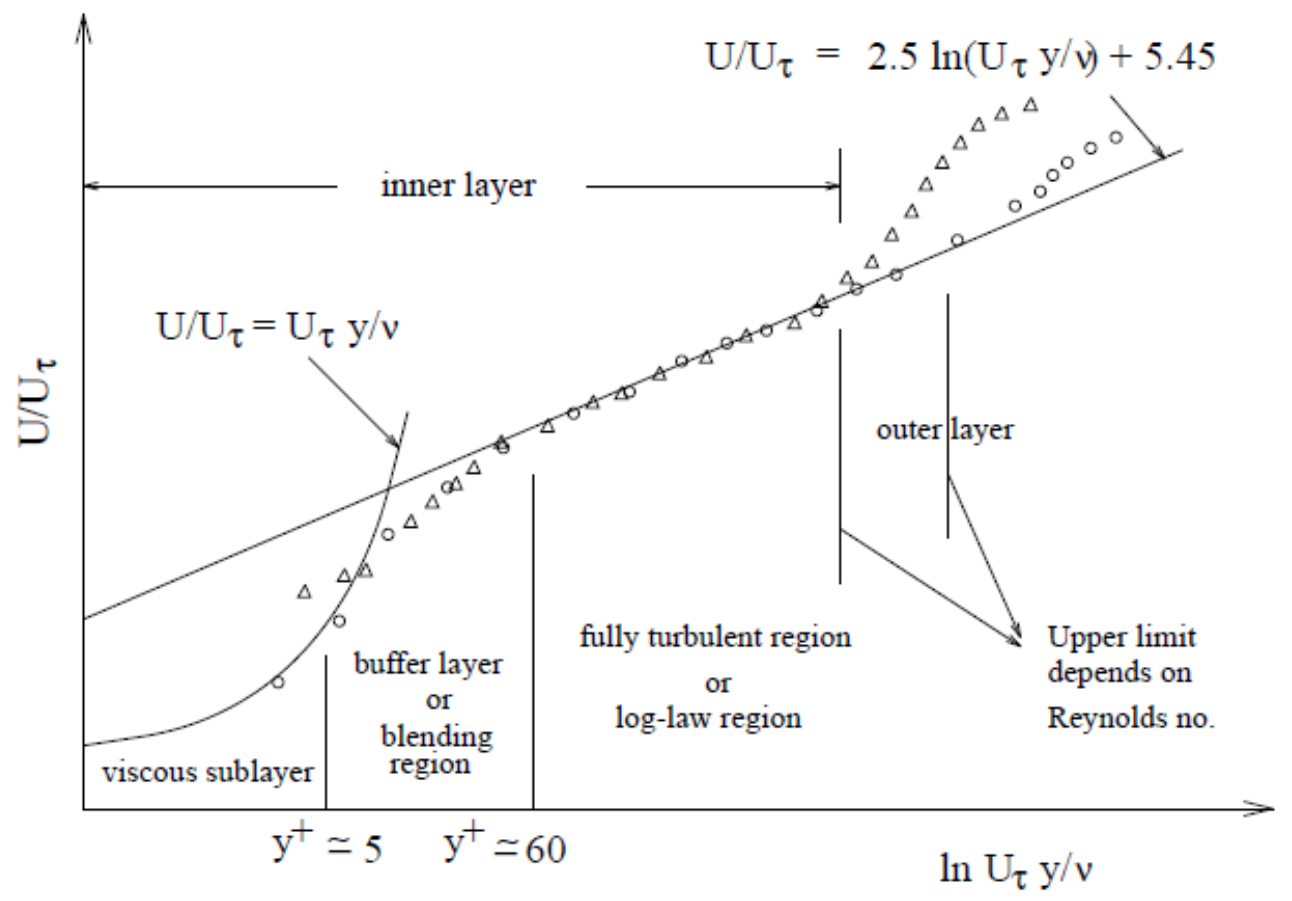

Figure 3.6.2: Illustration of the near-wall region (Wilcox 1993)

The standard wall functions that are used to capture the activity in the near-wall region are presented in the following equation set.

$$
\begin{gathered}
U^{+}=\frac{1}{\kappa} \ln \left(E y^{+}\right) \\
U^{+}=\frac{U_{p} C_{\mu}{ }^{1 / 4} K_{p}{ }^{1 / 2}}{\frac{\tau_{w}}{\rho}} \\
y^{+}=\frac{\rho C_{\mu}{ }^{1 / 4} K_{p}{ }^{1 / 2} y_{p}}{\mu}
\end{gathered}
$$

Where,

$\kappa=$ von Karman constant $(=0.42)$ 


$$
\begin{aligned}
& E=\text { Empirical constant }(=9.793) \\
& U_{p}=\text { Mean velocity of the fluid at point } \mathrm{P} \\
& K_{p}=\text { Turbulence kinetic energy at point } \mathrm{P} \\
& y_{p}=\text { Distance of point } \mathrm{P} \text { to the wall } \\
& \mu=\text { Dynamic viscosity of the fluid }
\end{aligned}
$$

\begin{tabular}{|c|c|}
\hline \multicolumn{2}{|c|}{ Mesh details } \\
\hline Type & Tetrahedral \\
\hline Scale factor & 0.4 \\
\hline Seed size & 0.5 \\
\hline Number of elements created & 1.4 million \\
\hline Value of $y^{+}$ & $30-60$ \\
\hline
\end{tabular}

Table 3.6.1: Details of the generated mesh

After this critical process, the inlet and the outlet boundaries are defined as velocity-inlet and pressure outlet respectively. The meshed geometry with the boundaries is presented in Fig 3.6.2.

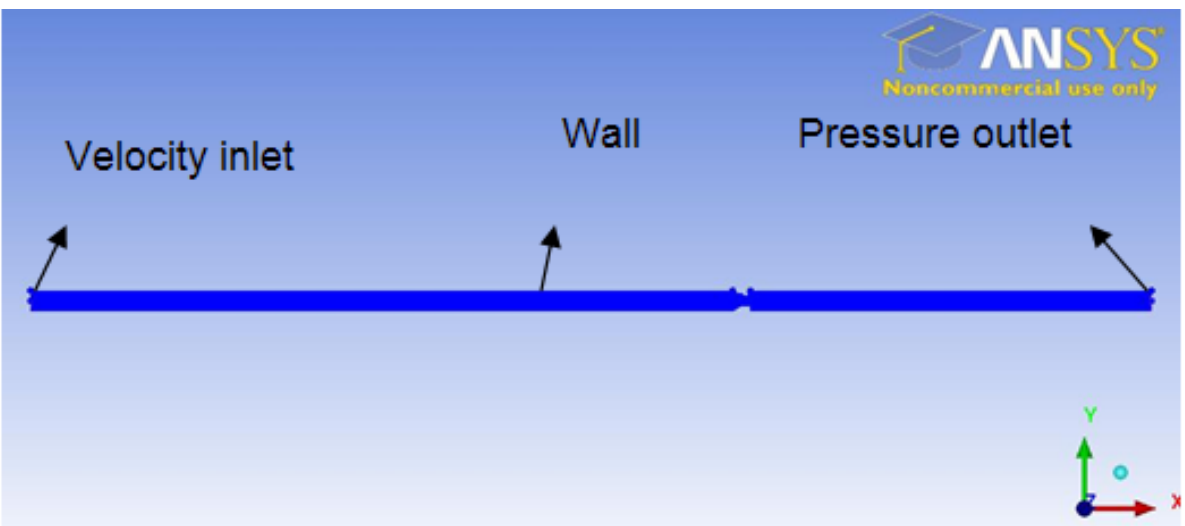

Figure 3.6.2: Boundaries surrounding the control volume 
The generated mesh is saved and is ready to be uploaded into the solver (FLUENT). The process of setting up the simulation in the solver software is discussed in detail in the following section.

\subsection{Solver}

The generated mesh was checked in FLUENT in order to estimate any discrepancies. Sometimes anomalous errors prop up due to faulty meshing. Fortunately, in our case this did not happen. Due to the presence of particles in the flow, achieving a solution through steady state solver is difficult. It has to be noted that an attempt to achieve a converged solution by employing a steady state solver was made, but the solution never converged. Thus an unsteady, implicit solver was selected. Since the mixture model is not compatible with the density based solver, a pressure based solver was selected. Since the $\mathrm{y}^{+}$value of the mesh in question was adjusted to a value between 30 and 60 , using the adapt option, the k-epsilon turbulence model with standard wall functions was activated to account for turbulent fluctuations. The mixture multi-phase model was activated to account for the particles present in the flow.

The material properties of the two phases in question were entered into solver. The properties of primary phase (xylene) and secondary phase (ADP) particles have already been presented in Table 3.5.1. FLUENT lets us specify the size of the secondary phase particles. The following step involves specifying the boundary conditions.

Since the mixture model treats the two-phase flow as a mixture, behavior of each entity namely; xylene, ADP particles, and the mixture itself have to be provided as inputs to the solving software. At the inlet, xylene which is the primary phase is provided with a velocity of $2 \mathrm{~m} / \mathrm{s}$. The initial guess for the secondary phase velocity can be any arbitrary value less than $2 \mathrm{~m} / \mathrm{s}$. Thus the secondary phase is provided with a velocity of $1.5 \mathrm{~m} / \mathrm{s}$. The initial volume fraction of the ADP particles is also provided. This value has to be less than 0.5 to satisfy the constraints of the 
mixture model. Turbulent intensity and hydraulic diameter values have to be provided as inputs for the mixture phase. Since we are dealing with a medium to high Reynolds number flow, turbulent intensity of $3.4 \%$ is assumed to be a good initial guess. The hydraulic diameter is specified as $0.0508 \mathrm{~m}$ (2 inches).

The outlet is kept at atmospheric pressure (1 atm). It is not required for us to provide any guesses for the primary phase at the outlet. However, for the secondary phase, backflow volume fraction is assumed to be same as the input value. This helps one achieve fully-developed flow conditions. For the mixture phase, we specify similar values of turbulent intensity and hydraulic diameter. What follows next can be deemed as the trickiest and the most excruciating part of the whole computational fluid dynamic process. The solving algorithm, convergence criterion, underrelaxation factors, time-step, and the number of iterations per time step have to be chosen in such a way that the solution converges steadily at each time step.

Initially, the solution was solved for a first order upwind discretization scheme. Later, it was solved using second order upwind discretization. All the presented results are for second order discretization scheme. It is highly recommended that one uses the pressure implicit with splitting of operators (PISO) algorithm for transient flow problems. This algorithm provides the coupling between pressure and velocity (FLUENT Inc 2009). Experience and careful scrutinization of the way the solution progresses is the only thing that can help one select the right under-relaxation factors. These values were adjusted ever so gently to produce a world of difference in the convergence process. The residuals of all the governing equations were set to $1 \mathrm{e}-05$ to obtain maximum accuracy. The time step and the number of time steps were adjusted to a particular value so that their product produced time in seconds that was greater than the total flow time. Since the pipe is approximately $2.8 \mathrm{~m}$ is length, and the fluid flows through it at a rate of $2 \mathrm{~m} / \mathrm{s}$, it is safe to assume that the flow time is close to 2 seconds. The velocity and the concentration profiles at 2.5 seconds, 3.5 seconds, and 4.5 seconds were compared, and it was found that there was no difference between them. Thus, the results are generated at 4.5 seconds to eliminate any 
dilemma of not achieving steady state, fully-developed flow conditions. The analysis that follows after the solution converges is termed as post-processing. The parameters mentioned above are presented in Table 3.7.1.

\begin{tabular}{|c|c|}
\hline Solver & Pressure based solver \\
\hline Algorithm & Pressure Implicit with Splitting Operators \\
& (PISO) \\
\hline Residuals & $1 \mathrm{e}-05$ \\
\hline Flow time & $4.5 \mathrm{~s}$ \\
\hline
\end{tabular}

Table 3.7.1: The parameters employed to simulate the multi-phase flow

\subsection{Post-processing}

After the end of each time step, FLUENT generates and stores a data file. This data file has the information of all the variables at each and every cell present in the volume mesh. One can gain useful information about the variation of the flow variables at any location in the control volume. FLUENT lets us create points, lines, and planes anywhere in the control volume. Thus one can predict how the variables are changing at a point, along a line, and/or in a particular plane. Organizing and interpreting the enormous amount of data is a gargantuan task. A dedicated effort was made to organize the reams of data obtained, and present them in a manner that is simplistic and systematic at the same time. 
CHAPTER IV

\section{RESULTS AND DISCUSSION}




\subsection{Introduction}

This section presents, in detail, the effect of various parameters: particle diameter, initial efflux concentration, and Reynolds number on the concentration profiles of the slurry at the fully developed region and the exit plane of the ASME flow nozzle. It is best to recognize the constants and variables that will be incorporated in this analysis. The Reynolds number of the flow, the initial particle efflux concentration, and the particle diameters are varied to investigate the effect of the geometric contraction on the concentration profiles. The concentration profiles for different cases are presented at the exit plane of the nozzle, and are compared with those at the fully-developed region of the pipe. Suitable reasoning to explain the variation of the concentration profiles at the exit plane is also presented. The different cases that are computed in this section are outlined in Table 4.1.1.

\begin{tabular}{|c|c|c|c|c|c|c|c|}
\hline \multicolumn{4}{|c|}{ Pipe Reynolds number, $R_{D}=140000$} & \multicolumn{4}{|c|}{ Pipe Reynolds number, $\operatorname{Re}_{D}=280000$} \\
\hline & Effll & ncen & $n(C)$ & & Effl & ncen & on $(C)$ \\
\hline $\begin{array}{l}\text { Small } \\
38 \mu m\end{array}$ & 0.05 & 0.2 & 0.3 & $\begin{array}{l}\text { Small } \\
38 \mu m\end{array}$ & 0.05 & 0.2 & 0.3 \\
\hline $\begin{array}{c}\text { Medium } \\
75 \mu \mathrm{m}\end{array}$ & 0.05 & 0.2 & 0.3 & $\begin{array}{c}\text { Medium } \\
75 \mu \mathrm{m}\end{array}$ & 0.05 & 0.2 & 0.3 \\
\hline $\begin{array}{l}\text { Large } \\
150 \mu \mathrm{m}\end{array}$ & 0.05 & 0.2 & 0.3 & $\begin{array}{l}\text { Large } \\
150 \mu \mathrm{m}\end{array}$ & 0.05 & 0.2 & 0.3 \\
\hline
\end{tabular}

\section{Table 4.1.1: Different cases studied}

It is best to validate the models that were employed to generate the profiles. This was done in the case of both the velocity and concentration profiles in the fully-developed region. The fully developed region is the location beyond which the velocity profiles do not change with 
the spatial coordinate system. In the process of defining the fully-developed region, a steady state simulation with just the primary phase was computed. The velocity profiles were plotted at regular intervals of $\mathrm{X} / \mathrm{D} \sim 10$ from the pipe inlet. The velocity profiles were compared at $\mathrm{X} / \mathrm{D}$ 10, X/ D 20, and X/ D 30. It was found that there was no appreciable difference in the velocity profiles at locations $\mathrm{X} / \mathrm{D} 20$ and $\mathrm{X} / \mathrm{D} \sim 30$. Thus, $\mathrm{X} / \mathrm{D} \sim 30$ was taken as the location where fully developed flow is achieved. The location of the fully-developed region in relation to the exit plane of the nozzle is presented in Fig 4.1.1.

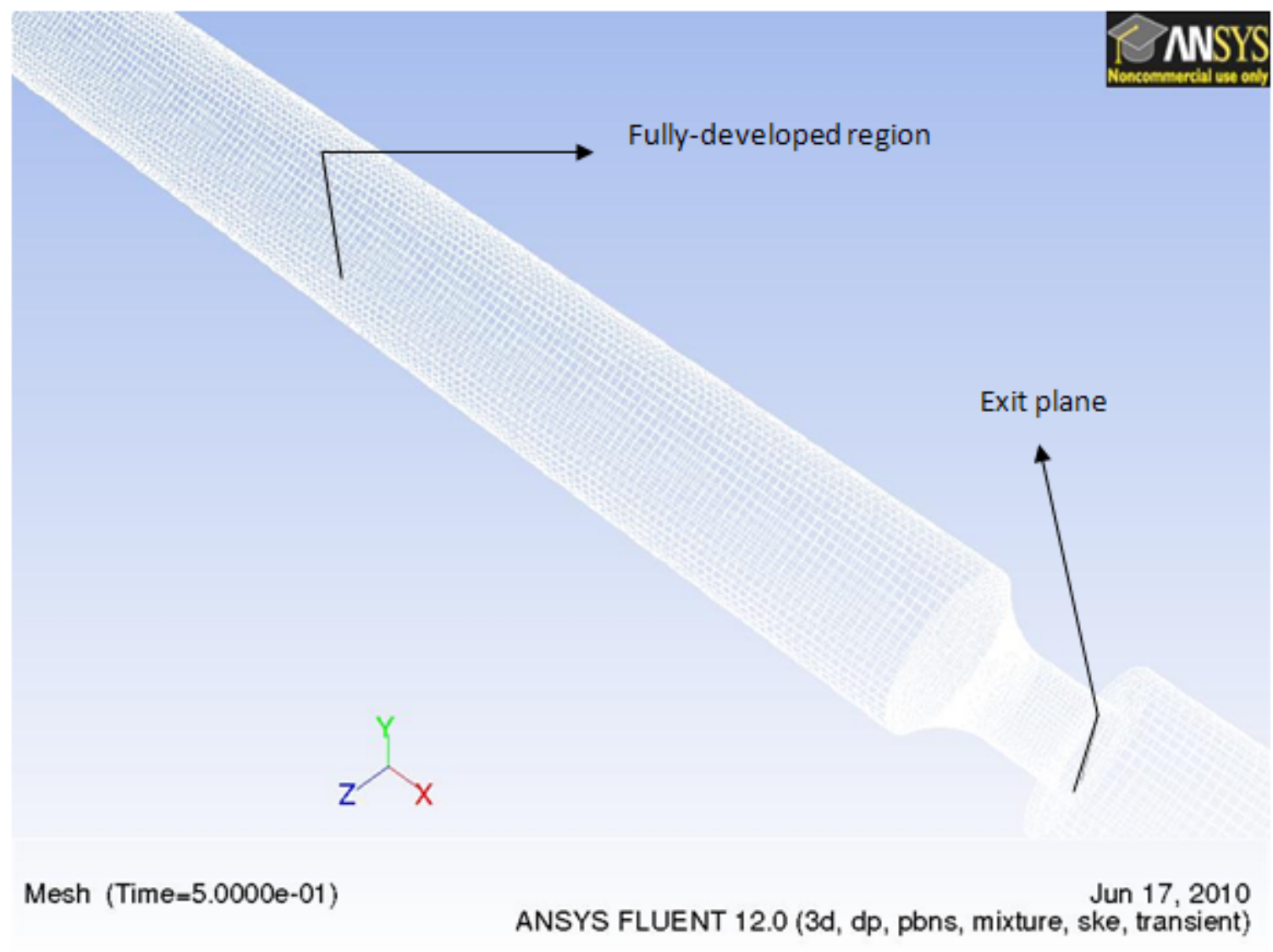

Figure 4.1.1: Position of fully-developed region with respect to the exit plane of the nozzle (Represented by dark vertical lines)

Fig 4.1.2 presents the exit plane with a greater degree of clarity. It is to be noted that the mesh has been coarsened and transformed to a hexahedral scheme so that one can clearly interpret the regions of interest. 


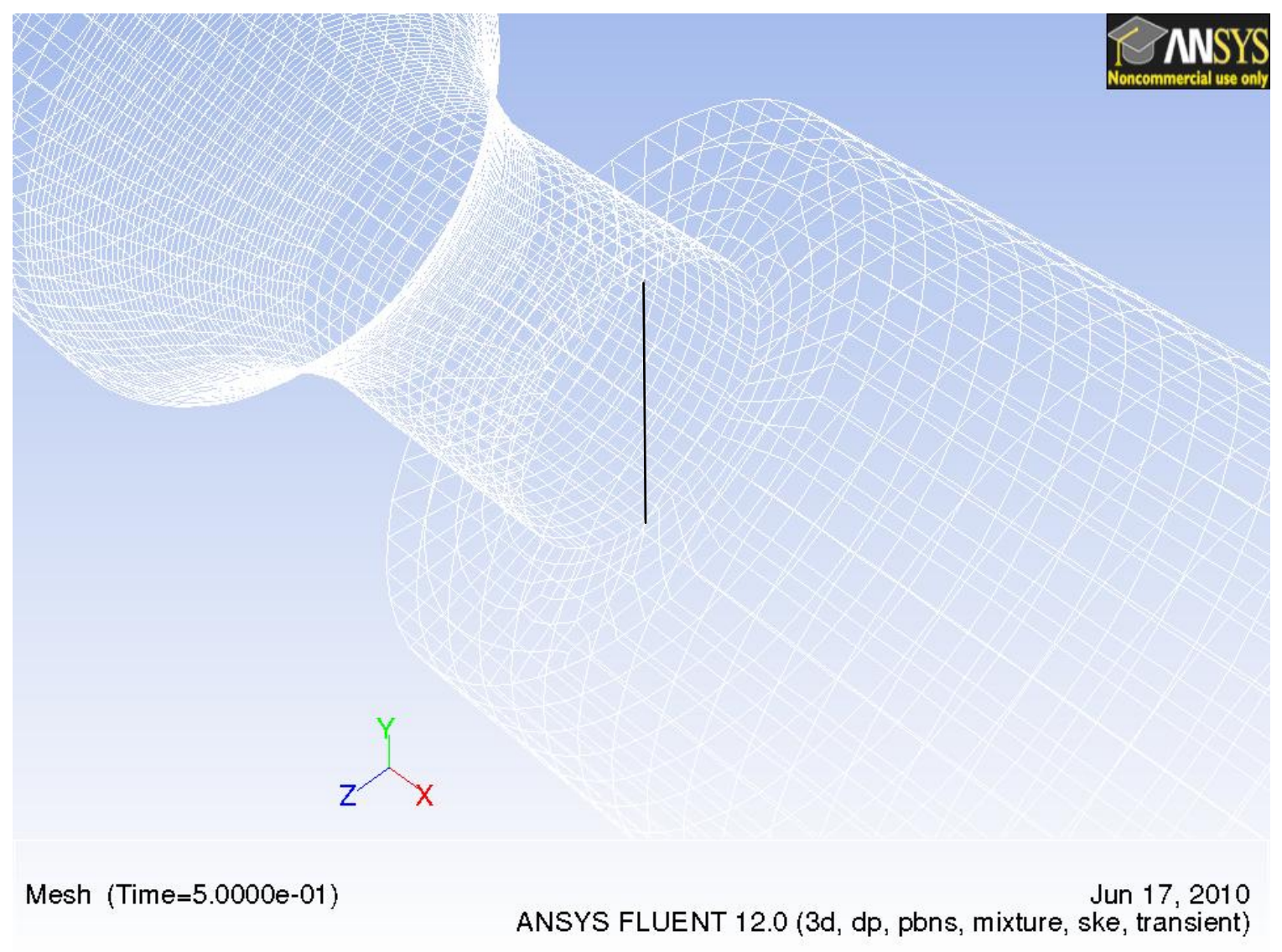

Figure 4.1.2: An enhanced view of the exit plane

The section of the chapter that follows is devoted to the velocity profiles. The profiles are first validated, and are later discussed.

\subsection{Velocity profiles and their validation}

This section talks briefly about the velocity profiles of the slurry flowing through a pipe. Since this work mainly concentrates on the concentration profiles of a slurry, the subject of velocity profiles is broached only to build confidence in the models that were used to simulate the flow. However, the velocity profiles of a two-phase flow, conveyed through a contraction, are compared to those found in the literature. The fully-developed velocity profile of xylene generated for two different grids is compared to the power law velocity profile in Fig 4.2.1. 


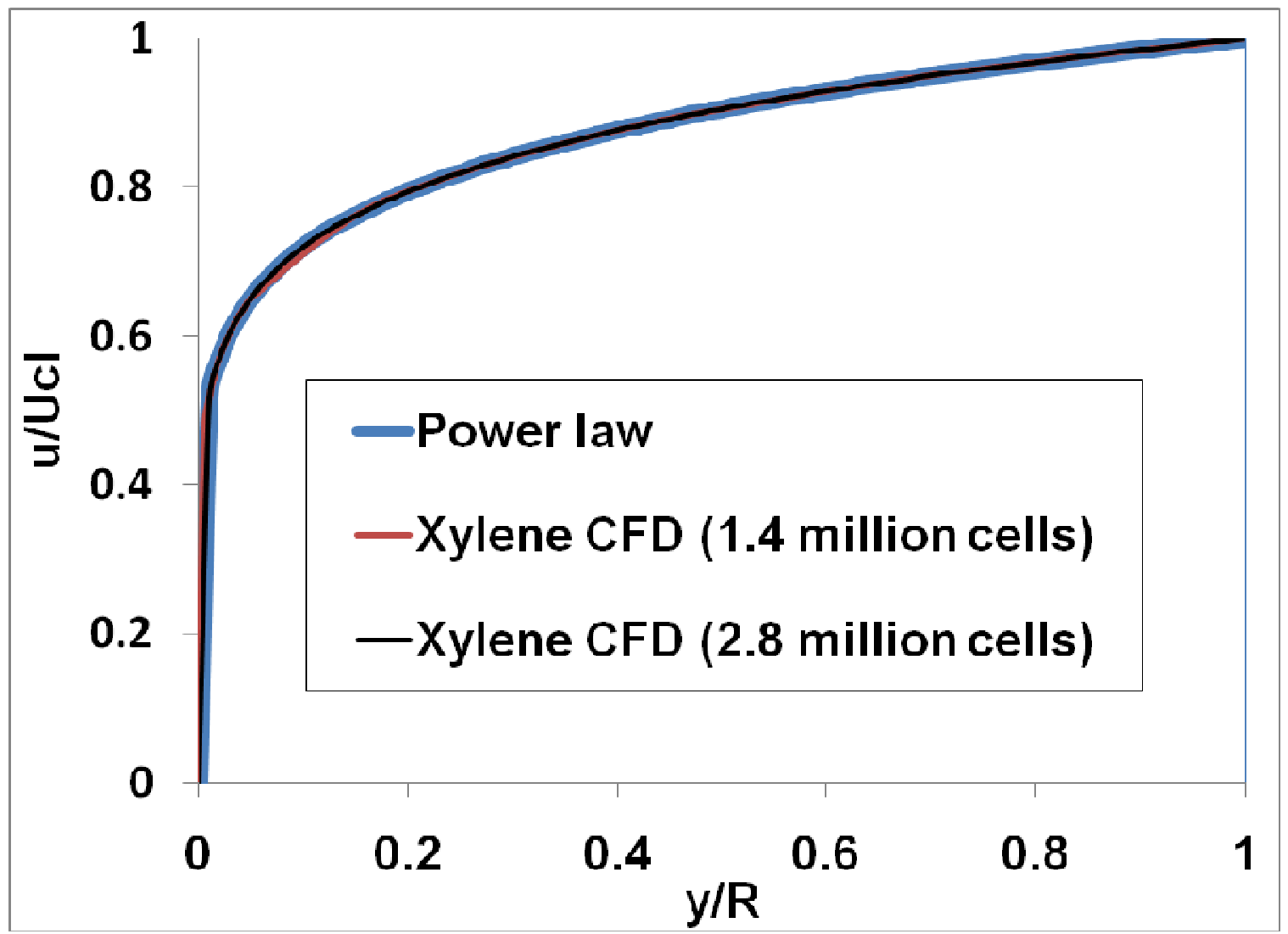

Figure 4.2.1: Power law profile compared with profiles generated through numerical computation

One can clearly observe that the velocity profile of xylene for the two different grids is in good accordance with the power law profile $(n=7)$. Thus, this is a clear indication that all the turbulence models are doing what they are supposed to do. It also implies that the solutions generated from here on are as close to grid independence as practically possible. In order to save valuable time, the computations will be performed using the coarser of the two grids mentioned in Fig. 4.2.1.

To check whether the turbulence models are functioning properly when the dispersed phase is introduced into the medium, the velocity profiles of the slurry are compared with those plotted by Wildman et al. (1992). As mentioned previously, Wildman et al. experimentally plotted velocity profiles of $5 \%$ silica gel slurry when it was conveyed through a contraction. The uncertainty in their velocity measurements is $\pm 0.5 \%$. Information regarding these plots is presented in Fig 2.1.7 and Fig 2.1.8. A similar exercise is conducted here. The velocity profiles of a $5 \%$ silica gel slurry 
are plotted when it was conveyed through the geometry that was described in Section 3.6. Velocity profiles at similar locations for a $5 \%$ silica gel were generated using the present computational model. They are plotted with the profiles generated by Wildman et al. in Fig 4.2.2.

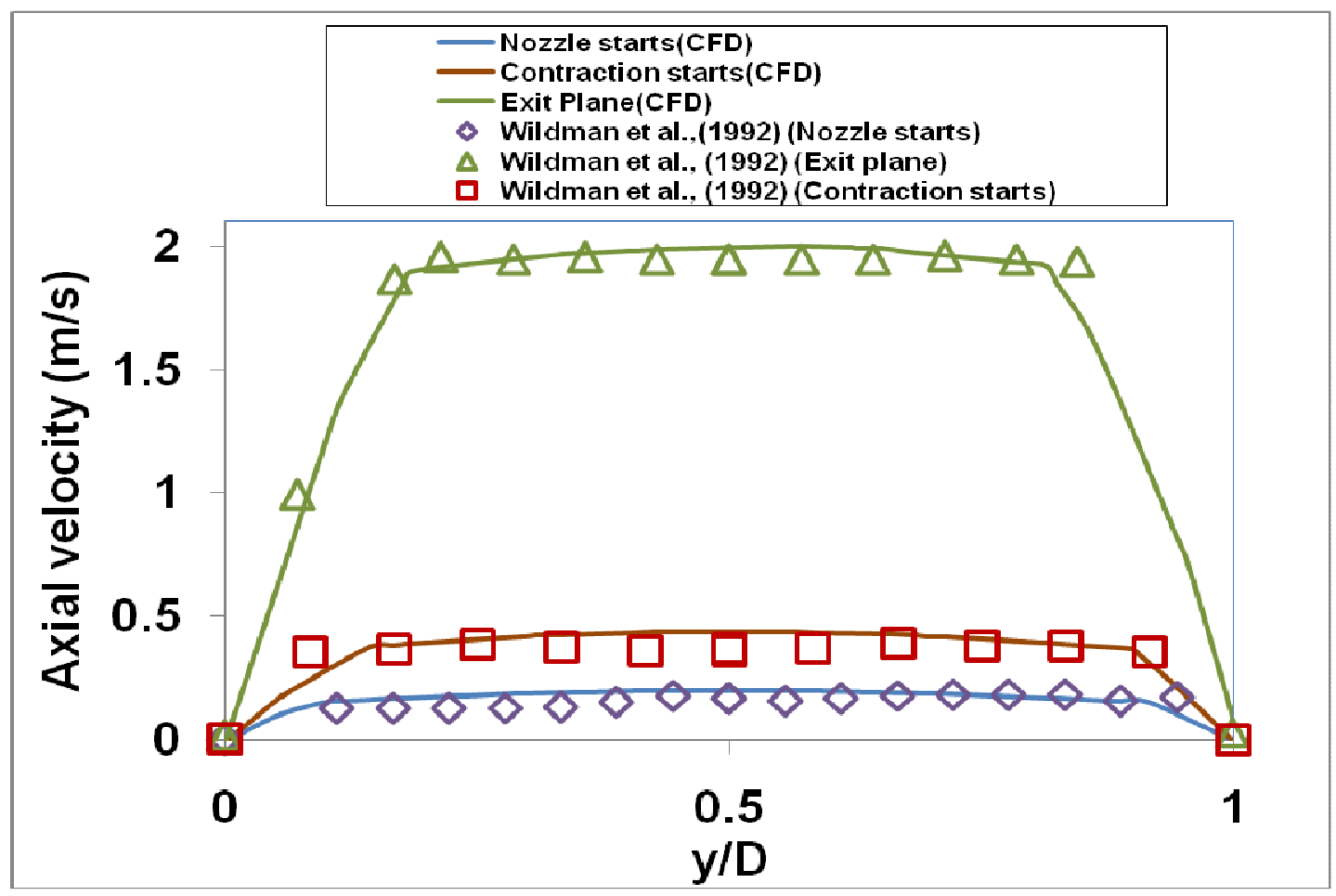

Figure 4.2.2: Comparison of velocity profiles of $5 \%$ silica gel slurry $\left(d_{p}=40 \mu \mathrm{m}\right)$ at pipe flow Reynolds number of $\left(\operatorname{Re}_{\mathrm{D}} \sim \mathbf{3 8 0 0}\right)$

There is a good agreement between the two velocity profiles. This is yet another testimony that our solving engine is doing a good job while predicting the velocity profiles of a single phase and a two-phase flow. Ekambara et al. (2009) performed numerical analysis to validate the experimental velocity profiles of a two-phase flow that were generated by Gillies et al. (2004). Ekambara et al. does not quantify the accuracy of their numerical simulations, but state that their model is most accurate for particle sizes less than $90 \mu \mathrm{m}$. Gillies et al. worked with a sand-water slurry where the average particle diameter was $90 \mu \mathrm{m}$. They operated at a pipe Reynolds number $\left(\mathrm{Re}_{\mathrm{D}}\right)$ of approximately 300000. Simulations incorporating the parameters used by Gillies et al. were performed using the current computational model. The comparison is presented in Fig 4.2.3. 


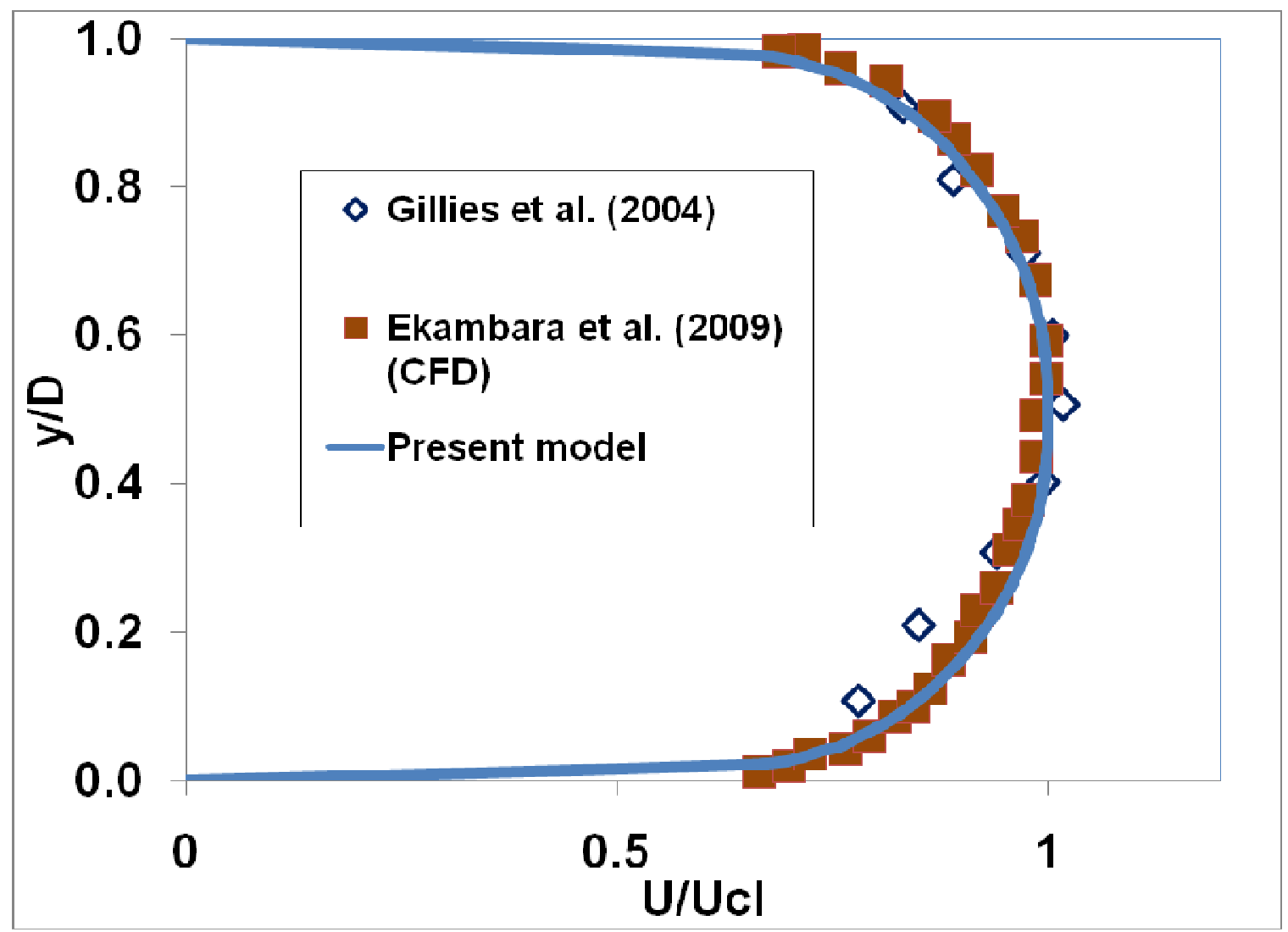

Figure 4.2.3: Comparison of velocity profiles of a sand-water slurry at a Reynolds number $\left(\operatorname{Re}_{\mathrm{D}}\right)$ of 300000

One can observe that the two computational results, the present model and Ekambara et al. (2009), are in fairly good agreement with each other. The variation between the experimental results (Gillies et al. (2004)) and the computational results, closer to the bottom wall, could be due to the presence of a fraction of particles larger than the mean particle diameter of $\left(d_{p}=90\right.$ $\mu \mathrm{m})$. There are deviations in the plots presented by Wildman et al. (1992) also; observe Fig 2.1.8 and Fig 4.2.2. Wildman et al. attributes these deviations to the slight imperfections on the surface of the wall. They also state that these imperfections make the experimental measurements very challenging in the regions closest to the wall. But, on the whole, the present computational model seems to work well in predicting the velocity profiles of a two-phase flow. So far so good, but our primary concern: the concentration profiles. How our model fares when working with concentration profiles is dealt with in the following sections. 


\subsection{Concentration profiles}

Initially, this section presents the validation of the computational model that was used to generate the data. Later, all the cases that were outlined in Table 4.1 .1 will be presented. Finally, a thorough discussion explaining the aforementioned cases will be presented. Before proceeding further it is best to illustrate a subtle difference between volume fraction and initial efflux concentration. Since FLUENT allows us to specify the inlet and the outlet volume fractions, it becomes essential for one to convert the initial efflux concentration value to its corresponding volume fraction value while setting up the simulation. This conversion is made possible with the help of the following equation set (Crowe et al. 1996):

$$
\begin{gathered}
\alpha_{d}+\alpha_{c}=1 \\
C_{w}=\frac{\rho_{d} \alpha_{d} \bar{u}}{\rho_{c} \alpha_{c} \bar{v}} \\
\rho_{m}=\rho_{c} \alpha_{c}+\rho_{d} \alpha_{d} \\
C=\frac{C_{w} \rho_{m}}{\rho_{d}}
\end{gathered}
$$

Where,

$$
\begin{array}{ll}
C_{w} & =\text { Concentration by weight } \\
\rho_{d} & =\text { Density of dispersed phase, }\left(\mathrm{Kg} / \mathrm{m}^{3}\right) \\
\rho_{c} & =\text { Density of continuous phase, }\left(\mathrm{Kg} / \mathrm{m}^{3}\right) \\
\bar{u} & =\text { Average velocity of secondary phase, }(\mathrm{m} / \mathrm{s})
\end{array}
$$




\subsubsection{Validation of the concentration profiles}

In order to eliminate any apprehension relative to the accuracy of the solution, two simulations for $38 \mu \mathrm{m}$ ADP particles at a primary phase Reynolds number of $\left(\operatorname{Re}_{\mathrm{D}}=140000\right)$ were simulated. The first simulation was run with a tetrahedral grid with 1.4 million cells, and the second was run with 2.8 million cells. The profiles were plotted on the same graph to enhance comparison. They are presented in Fig 4.3.1.6.

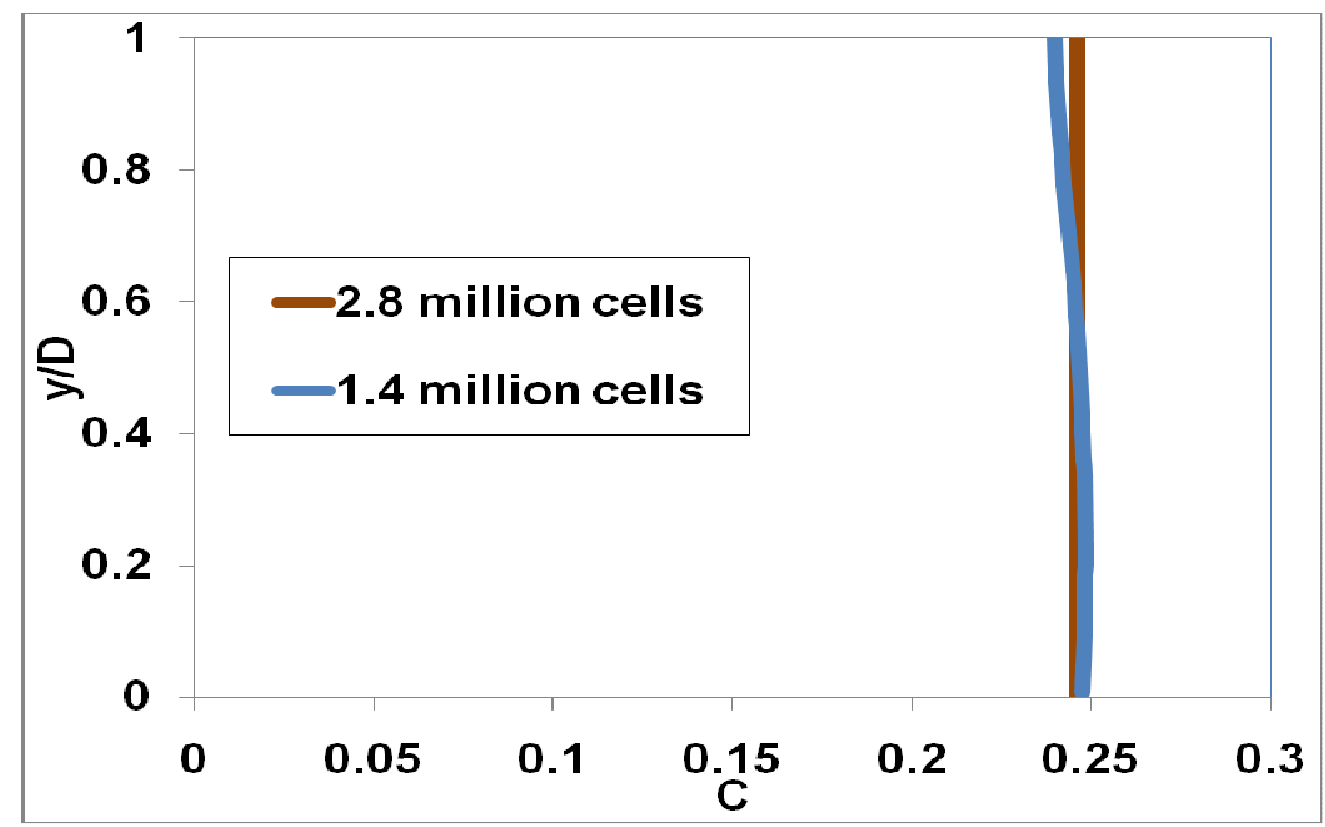

Figure 4.3.1.1: Concentration profiles of a xylene-ADP slurry $\left(d_{p}=38 \mu \mathrm{m}\right)$ at 0.25 efflux concentration computed with different cell volumes at $\left(\operatorname{Re}_{D}=140000\right)$

One can notice that while the velocity profiles are in good agreement for the two different meshes employed; see Fig 4.2.1, there is a maximum error of approximately $2 \%$ when the concentration profiles are compared. This anomaly might be explained by the variation in the value of $\mathrm{y}^{+}$. In the two meshes that were generated, the value of $\mathrm{y}^{+}$was adjusted to lie between 30 
and 60 by using the adapt function of FLUENT. However, several attempts to achieve the same $\mathrm{y}^{+}$value in case of both the meshes proved to be counterproductive. Another reason for this slight deviation in the concentration profiles could also be attributed to the unstructured nature of the mesh. Though unstructured grids display higher flexibility for automatic generation of grids around complex geometries, they sometimes give rise to slightly distorted cells. And these distorted cells slightly influence the accuracy of the solution (Hirsch 2007). Moreover, one cannot be certain whether it was the coarse or the fine grid that generated this so called distorted cell upon adaptation in FLUENT because the velocity profiles generated by both the meshes are in good agreement with each other. Thus, it is possible that the concentration profiles generated by the mixture multi-phase model are sensitive to the variation in the value of $\mathrm{y}^{+}$, or the presence of slightly distorted cells near the top wall. It has to be noted that the grid independence study was possible only for the smallest particles. When the finer mesh was used for the larger particles, the solution failed to converge. Perhaps, the finer mesh is not compatible with larger particles. The reasons for this occurrence are still being investigated.

The turbulence kinetic energy in the fully-developed region for a xylene-ADP slurry containing $38 \mu \mathrm{m}$ particles was compared for the fine and the coarse meshes employed. The plots are presented in Fig 4.3.1.2.

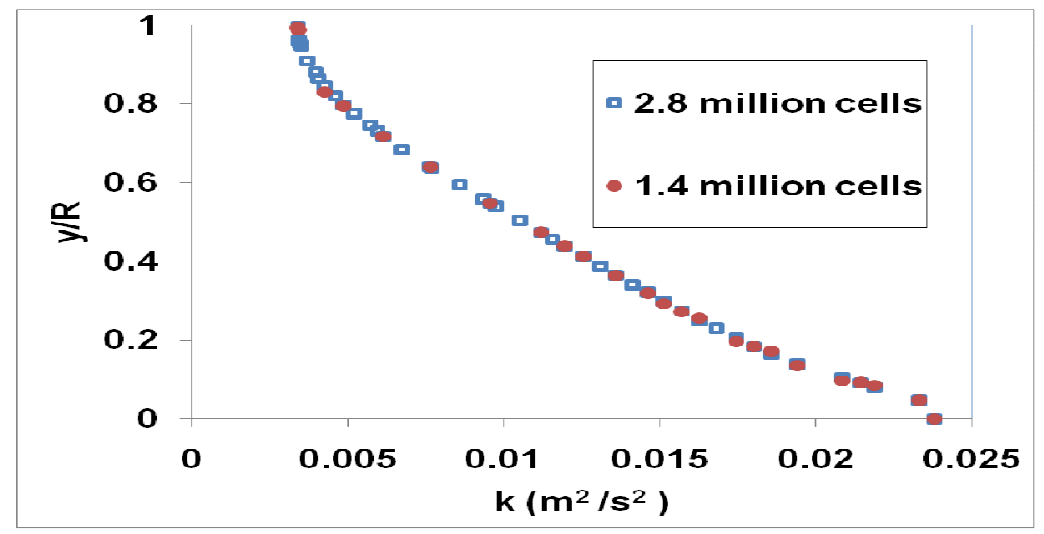

Figure 4.3.1.2: Turbulence kinetic energy profiles of a xylene-ADP slurry $\left(d_{p}=38 \mu \mathrm{m}\right)$ at 0.25 efflux concentration computed with different cell volumes at $\left(\operatorname{Re}_{\mathrm{D}}=\mathbf{1 4 0 0 0 0}\right)$ 
Since the velocity profiles and the turbulence kinetic energy profiles in case of the two grids are in good agreement with each other, and in order to save valuable time, the grid with 1.4 million cells is selected. It is to be noted that both the grids, 1.4 million cells and 2.8 million cells, satisfy all the constraints of the k-epsilon model with standard wall functions.

Experimental data relative to the concentration profiles of a slurry flowing through a pipeline is available in the open literature. The experimental data that had all the variables and constants clearly defined was selected for the purpose of validation. Gillies et al. (2004) generated experimental data in relation to sand water slurries. They plotted concentration profiles at $\mathrm{Re}_{\mathrm{D}}=300000$ for sand particles having a mean particle diameter of $90 \mu \mathrm{m}$. Experimental data was generated for initial volumetric concentrations of $19,24,29$, and $33 \%$. Their findings are presented in Fig 4.3.1.1. Computational simulations were run using exactly the same parameters that were employed by Gillies et al. The mesh with 1.4 million cells was used as it produced good results at higher Reynolds numbers while validating the velocity profiles. Since the Reynolds number employed by Gillies et al. is very close to the maximum Reynolds number that will be employed in this study, the 1.4 million mesh may be considered adequate to resolve the aforementioned flow regime. The comparison between the two data sets will be presented momentarily.

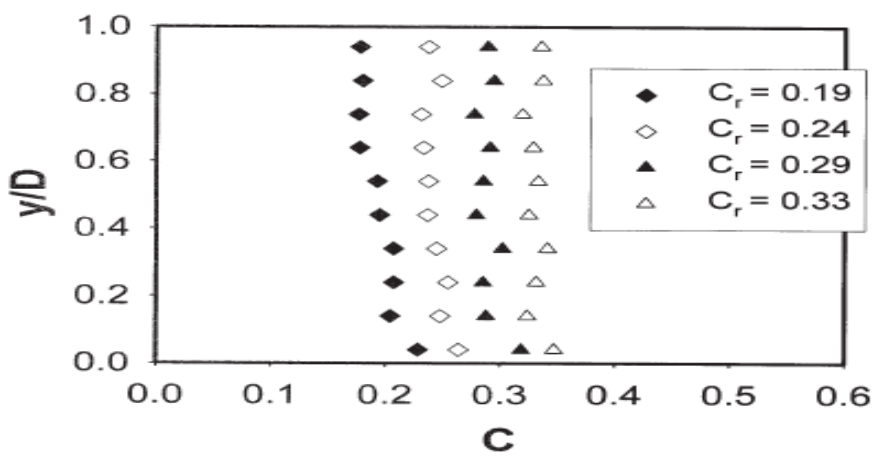

Figure 4.3.1.3: Concentration profiles of sand-water slurry at a $\operatorname{Re}_{\mathrm{D}}=\mathbf{3 0 0 0 0 0}$ (Gillies et al. 2004) 
The following figures contain both data sets plotted in a single graph to have a better understanding.

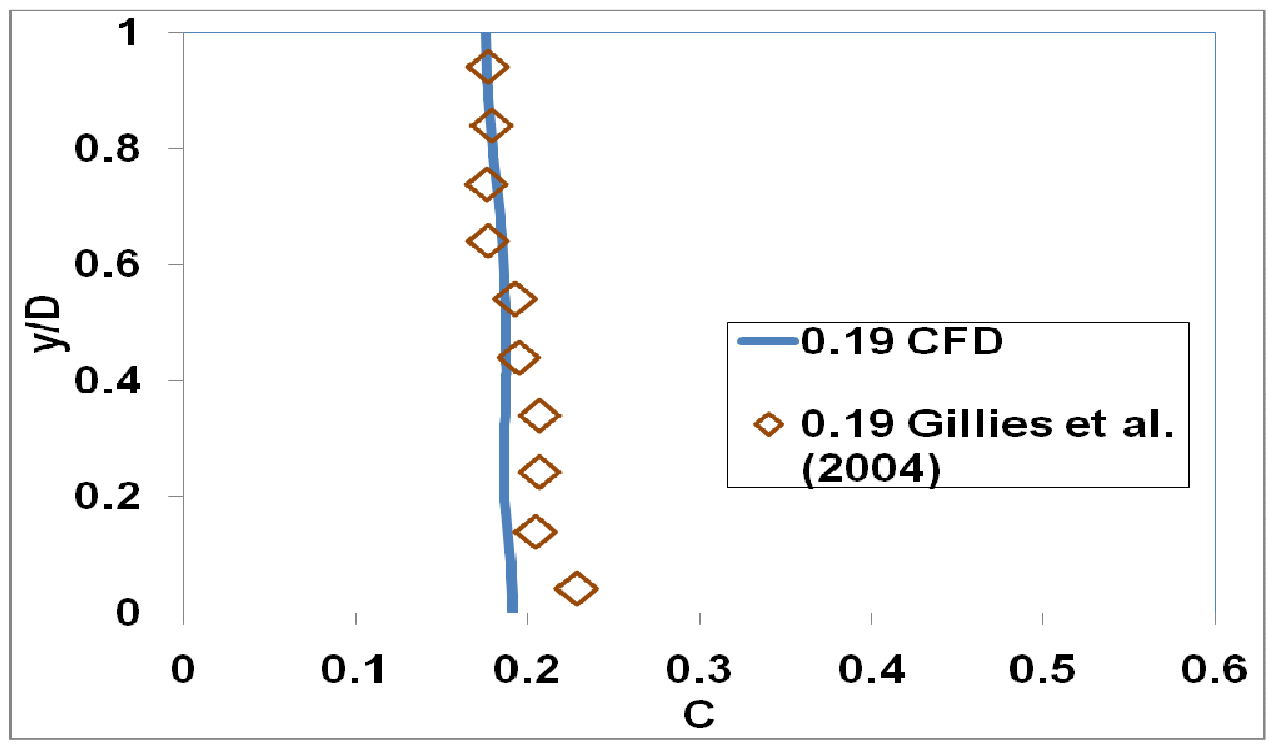

Figure 4.3.1.4: Comparison of concentration profiles of sand-water slurry at initial volumetric concentration of 0.19

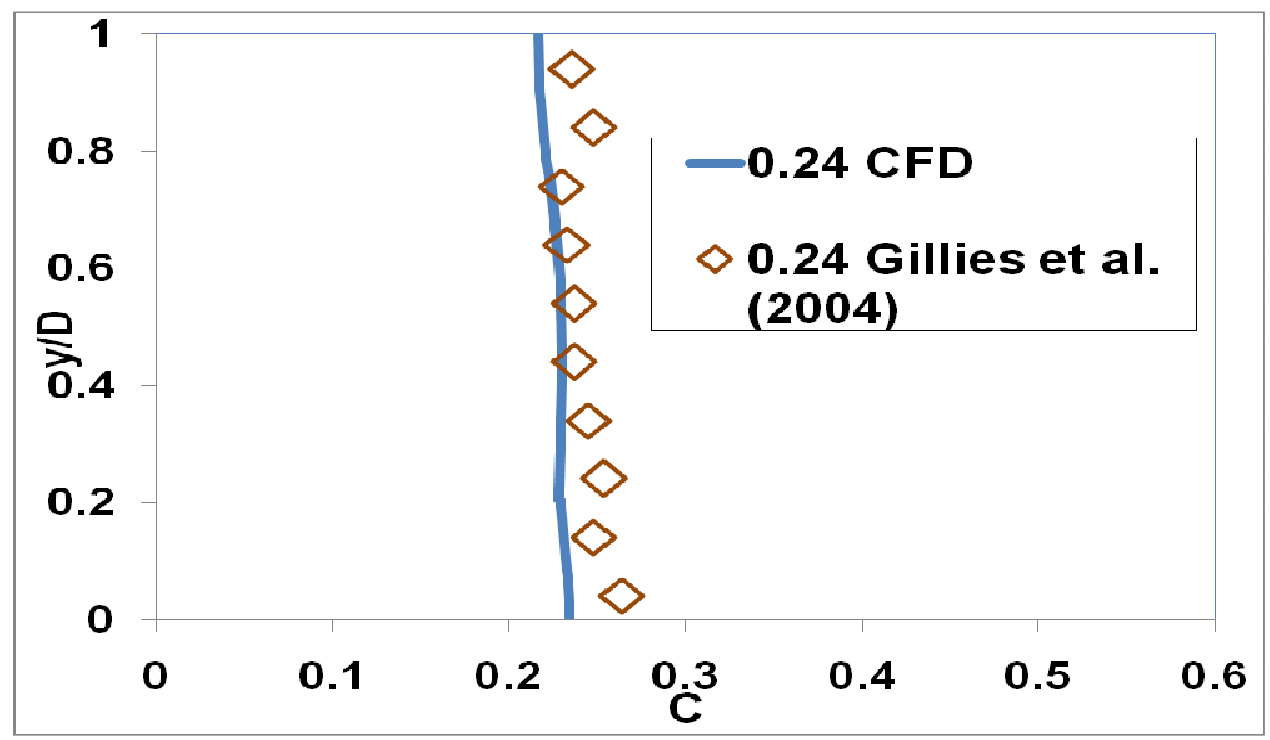

Figure 4.3.1.5: Comparison of concentration profiles of sand-water slurry at initial volumetric concentration of $\mathbf{0 . 2 4}$ 


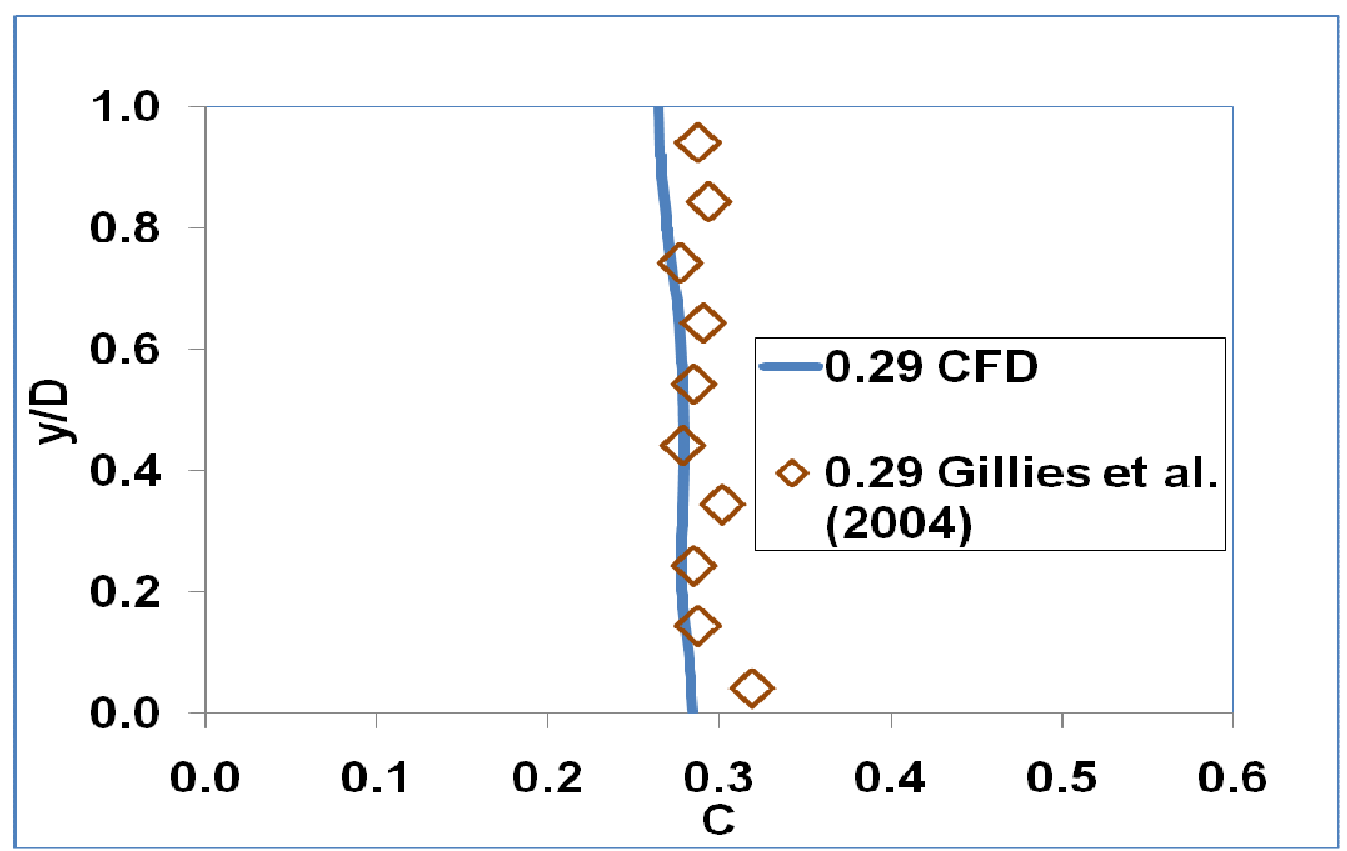

Figure 4.3.1.6: Comparison of concentration profiles of sand-water slurry at initial volumetric concentration of 0.29

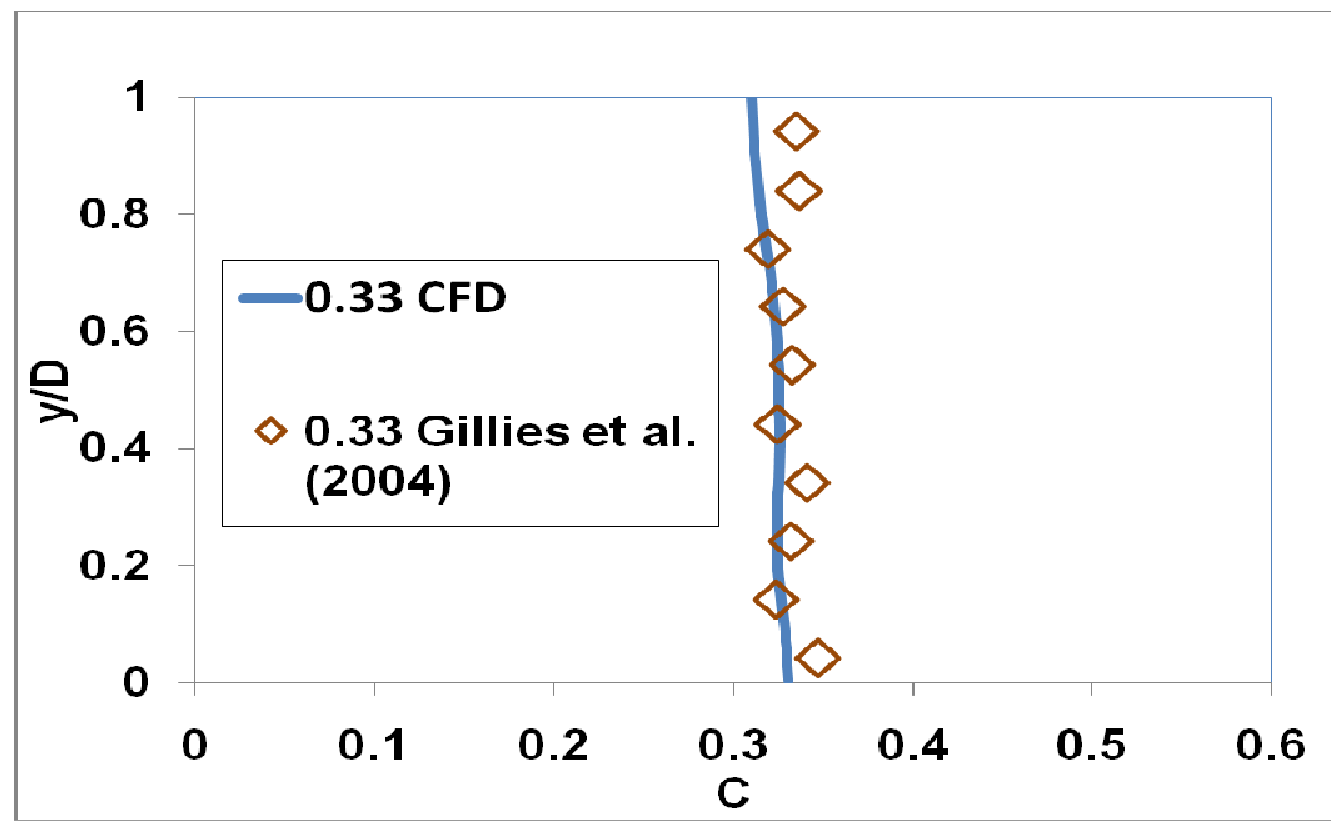

Figure 4.3.1.7: Comparison of concentration profiles of sand-water slurry at initial volumetric concentration of $\mathbf{0 . 3 3}$

After observing the graphs from Fig 4.3.1.3 through Fig 4.3.1.6, one can say that the present computational fluid dynamic model does an acceptable job while predicting the concentration 
profiles. A slight deviation at the top and bottom of the pipe is explained by Roco and Shook (1984). According to them, a computational model assumes the particles to be of uniform size. But in reality, there are small fractions of particles with dimensions smaller and larger than the average size. Explicitly, the particles used in the experiments are not mono-dispersed. Owing to this the computational data deviates from its experimental counterpart. The deviations could be also attributed to sensitivity of the mixture multi-phase model to predict the concentration profiles in the near-wall region. Roco and Shook worked with sand-water slurries where the mass median diameter ranged from $165 \mu \mathrm{m}$ to $520 \mu \mathrm{m}$. Further, Gillies et al. states that the experimental error is maximum closest to the walls.

From the above discussion it is clear that the present computational model is doing a satisfactory job while predicting the concentration profiles of a slurry. And thus, it is fair to say that all the results presented from here onwards can be considered substantially accurate. The different cases that were described in Table 4.1.1 will be analyzed next.

\subsubsection{Concentration profiles for $\left(\operatorname{Re}_{\mathrm{D}}=140000\right)$}

The concentration profiles for all the cases that belong to $\mathrm{Re}_{\mathrm{D}}=140000$ class will be presented and discussed in this section. The profiles will be presented in the increasing order of particle size and initial efflux concentration. Every figure will compare the concentration profiles registered at the fully-developed region and the exit plane of the geometry in question. These locations presented in Fig 4.1.1. The profiles are plotted along the dark vertical lines that appear in the figures. 


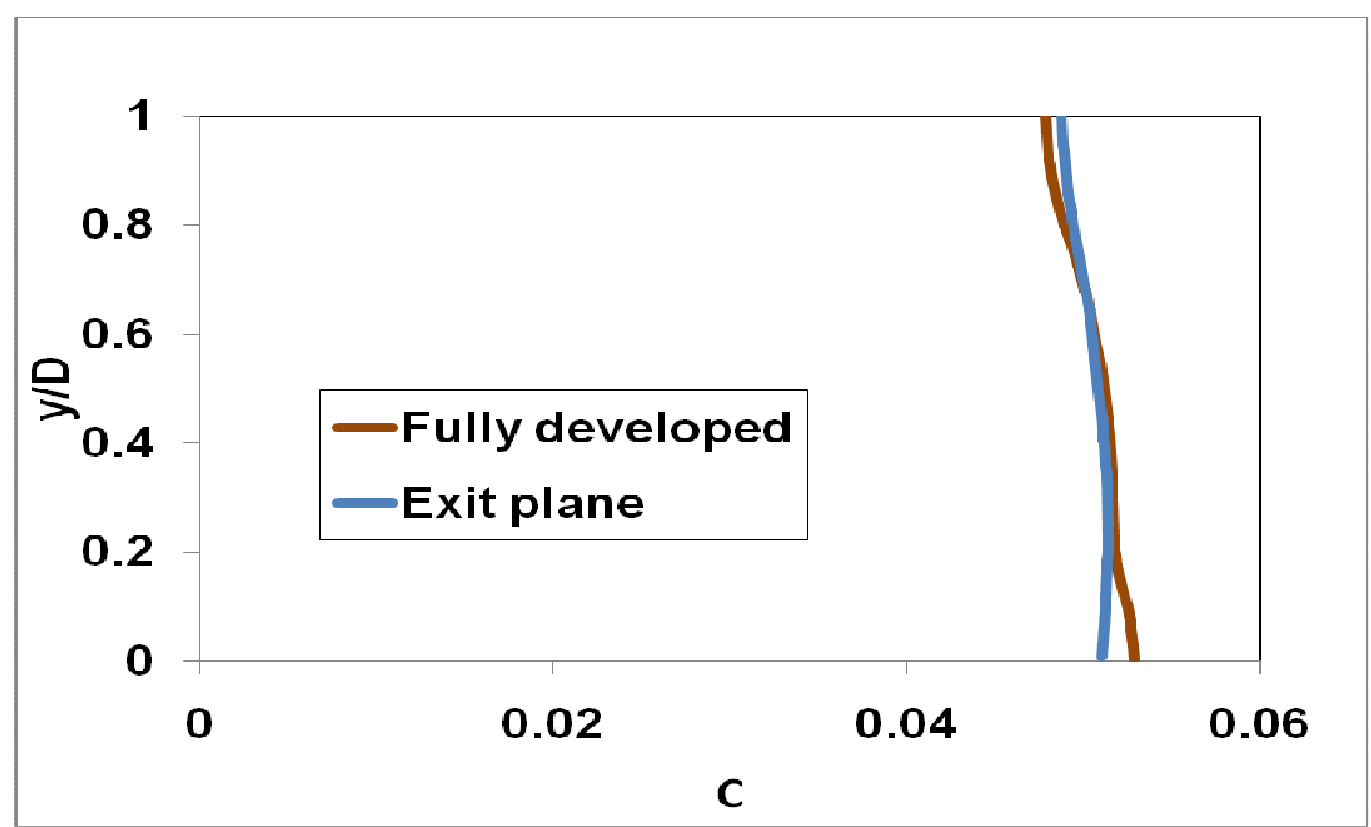

Figure 4.3.2.1: Concentration profiles of $38 \mu \mathrm{m}$ ADP particles at 0.05 initial efflux concentration as realized at the fully developed region and the exit plane

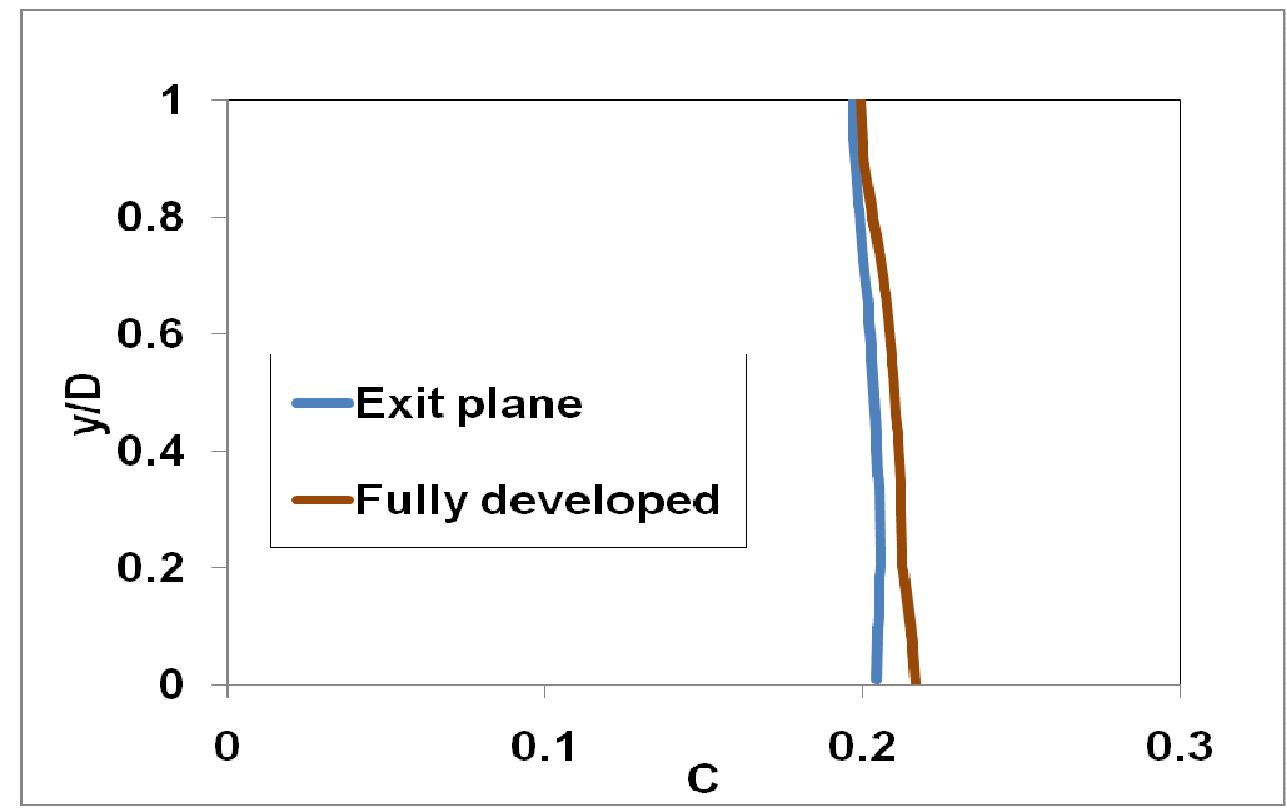

Figure 4.3.2.2: Concentration profiles of $38 \mu \mathrm{m}$ ADP particles at 0.2 initial efflux concentration as realized at the fully developed region and the exit plane 


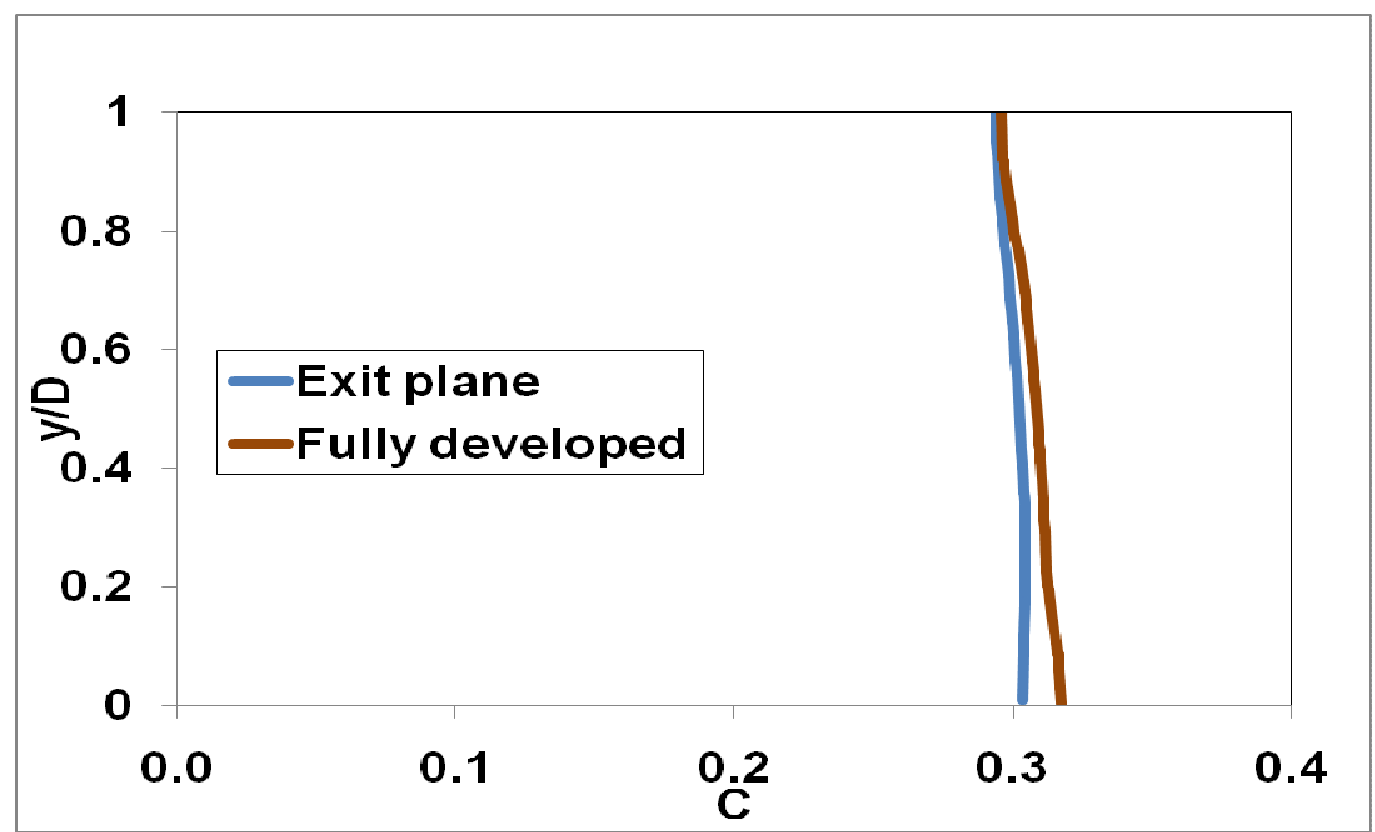

Figure 4.3.2.3: Concentration profiles of $38 \mu \mathrm{m}$ ADP particles at 0.3 initial efflux concentration as realized at the fully developed region and the exit plane

In Fig 4.3.2.1 through 4.3.2.3, the shape of the profile displays a slight slope at the fully developed region, the profile at the exit plane remains comparatively uniform; moreover, it is shifted closer to the initial efflux concentration value. Is this effect consistent with variation in particle size (coarser particles) is a question that will be answered after observing the profiles for $75 \mu \mathrm{m}$ particles. Figures 4.3.2.4 through Fig 4.3.2.6 represent the profiles of $75 \mu \mathrm{m}$ particles at different efflux concentrations. 


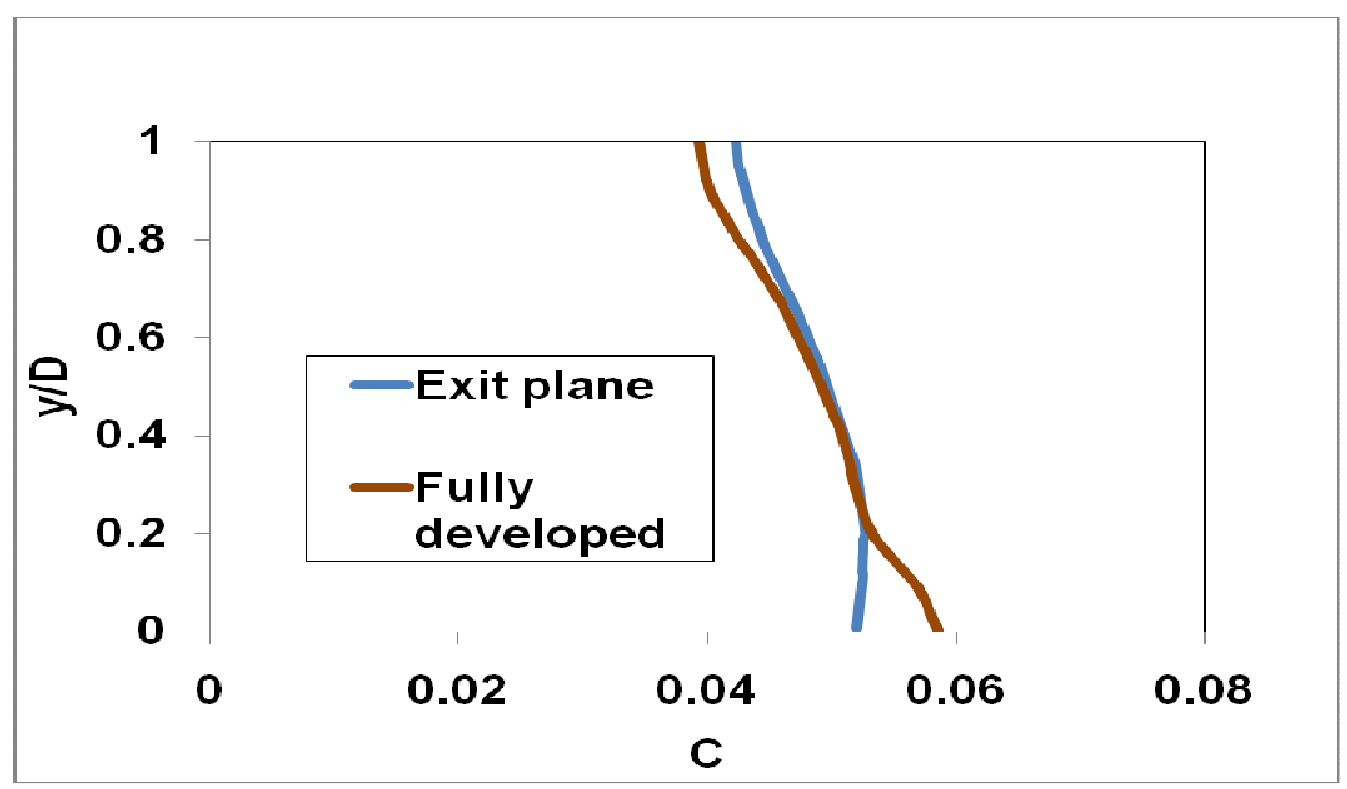

Figure 4.3.2.4: Concentration profiles of $75 \mu \mathrm{m}$ ADP particles at 0.05 initial efflux concentration as realized at the fully developed region and the exit plane

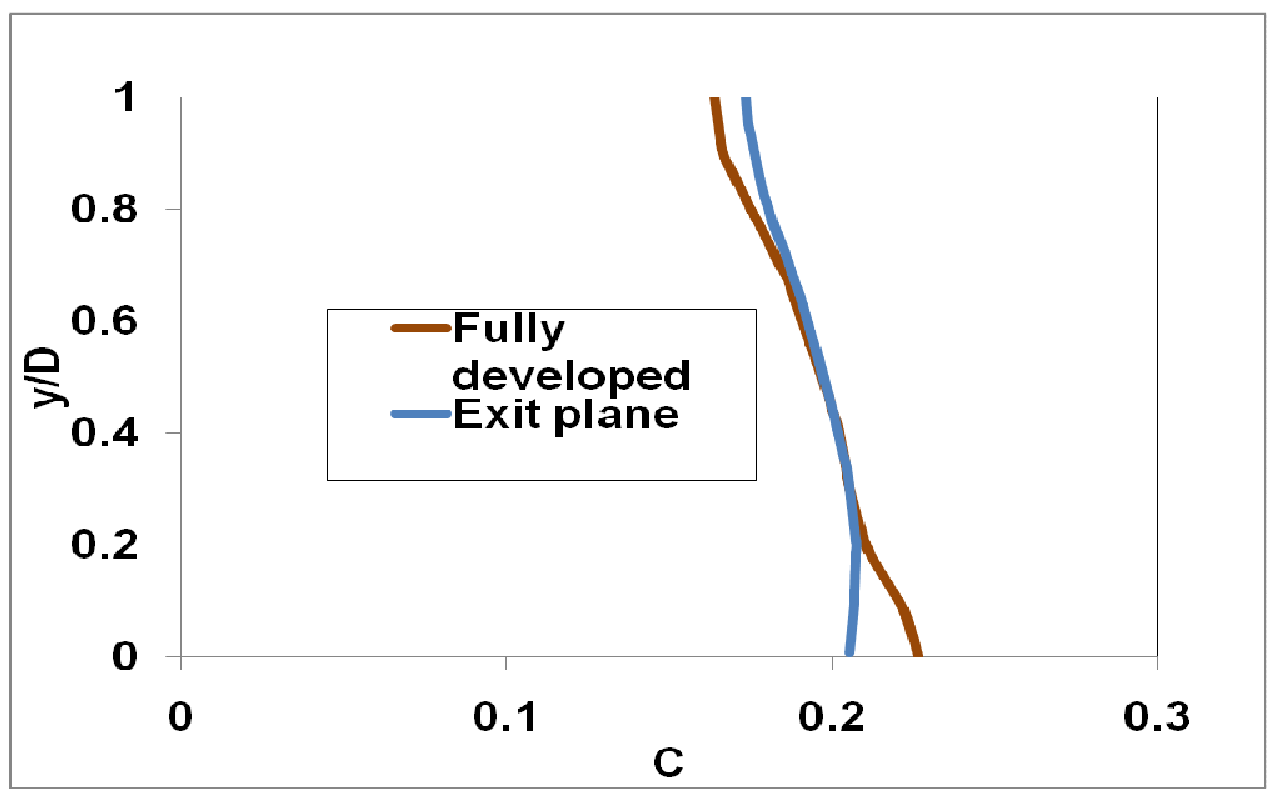

Figure 4.3.2.5: Concentration profiles of $75 \mu \mathrm{m}$ ADP particles at 0.2 initial efflux concentration as realized at the fully developed region and the exit plane 


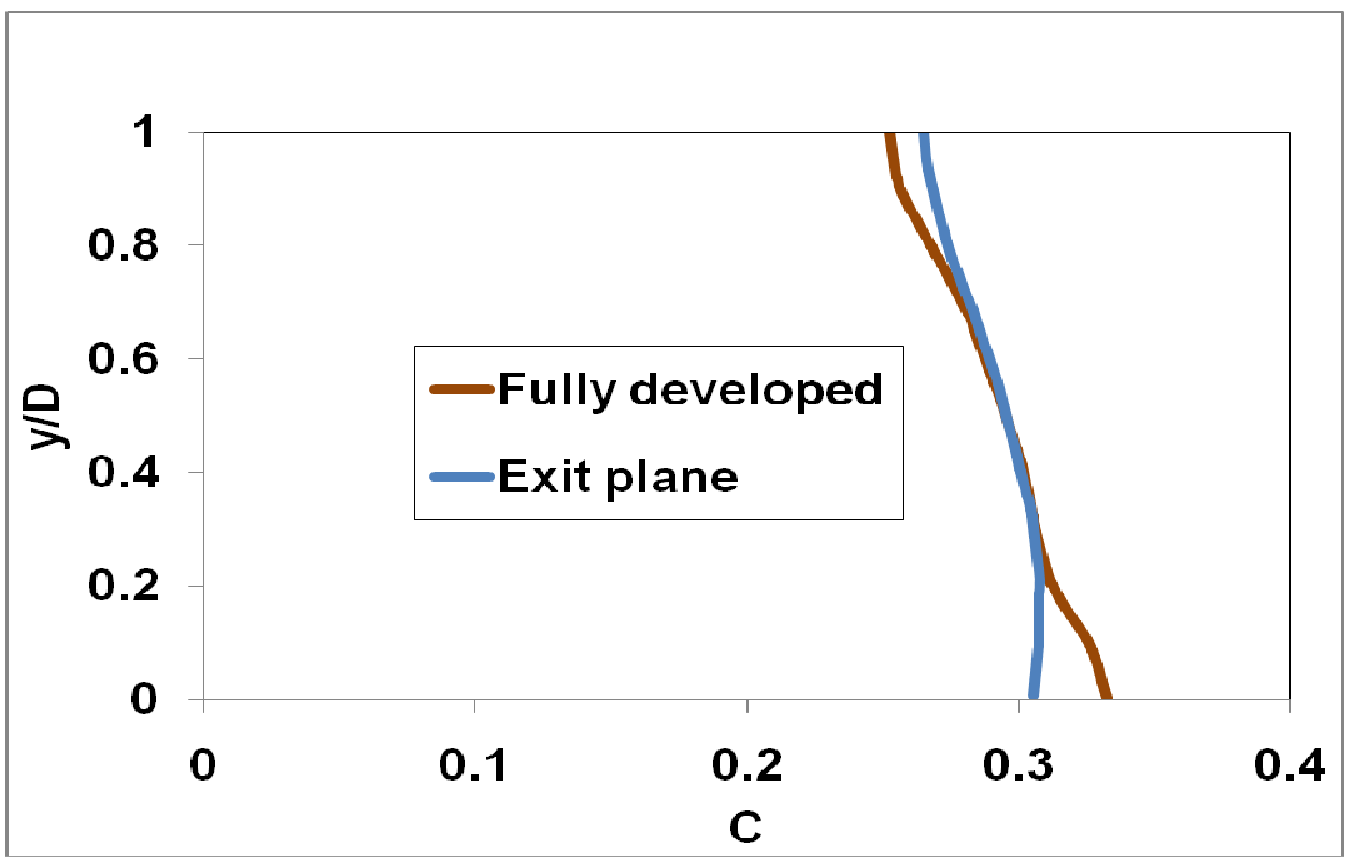

Figure 4.3.2.6: Concentration profiles of $75 \mu \mathrm{m}$ ADP particles at 0.3 initial efflux concentration as realized at the fully developed region and the exit plane

Owing to the larger size of the particles; the profiles succumb to the gravity effect. Hence, all the profiles at the fully developed region for the $75 \mu \mathrm{m}$ particles display a distinct slant. After observing the concentration profiles at the exit plane, one can observe that the profiles have yet again shifted closer to the initial efflux concentration, and display a greater degree of uniformity. Before concluding that this trend is consistent with the increase in particle size, the simulations are performed with a bigger particle size. The particle size is doubled, i.e., increased to $150 \mu \mathrm{m}$, and similar computational analysis is performed. The results from this analysis are presented in the series of plots that follow. 


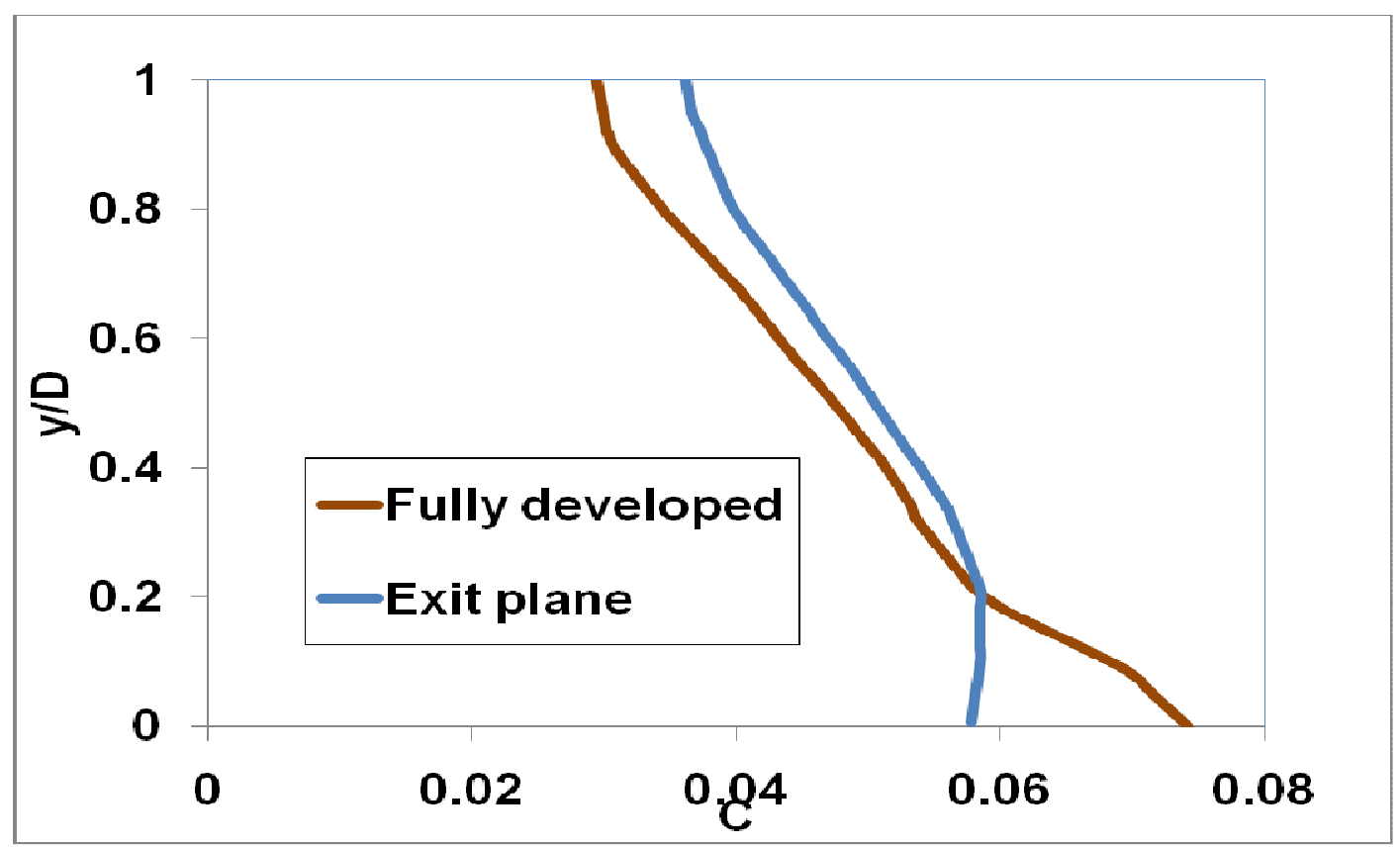

Figure 4.3.2.7: Concentration profiles of $150 \mu \mathrm{m}$ ADP particles at 0.05 initial efflux concentration as realized at the fully developed region and the exit plane

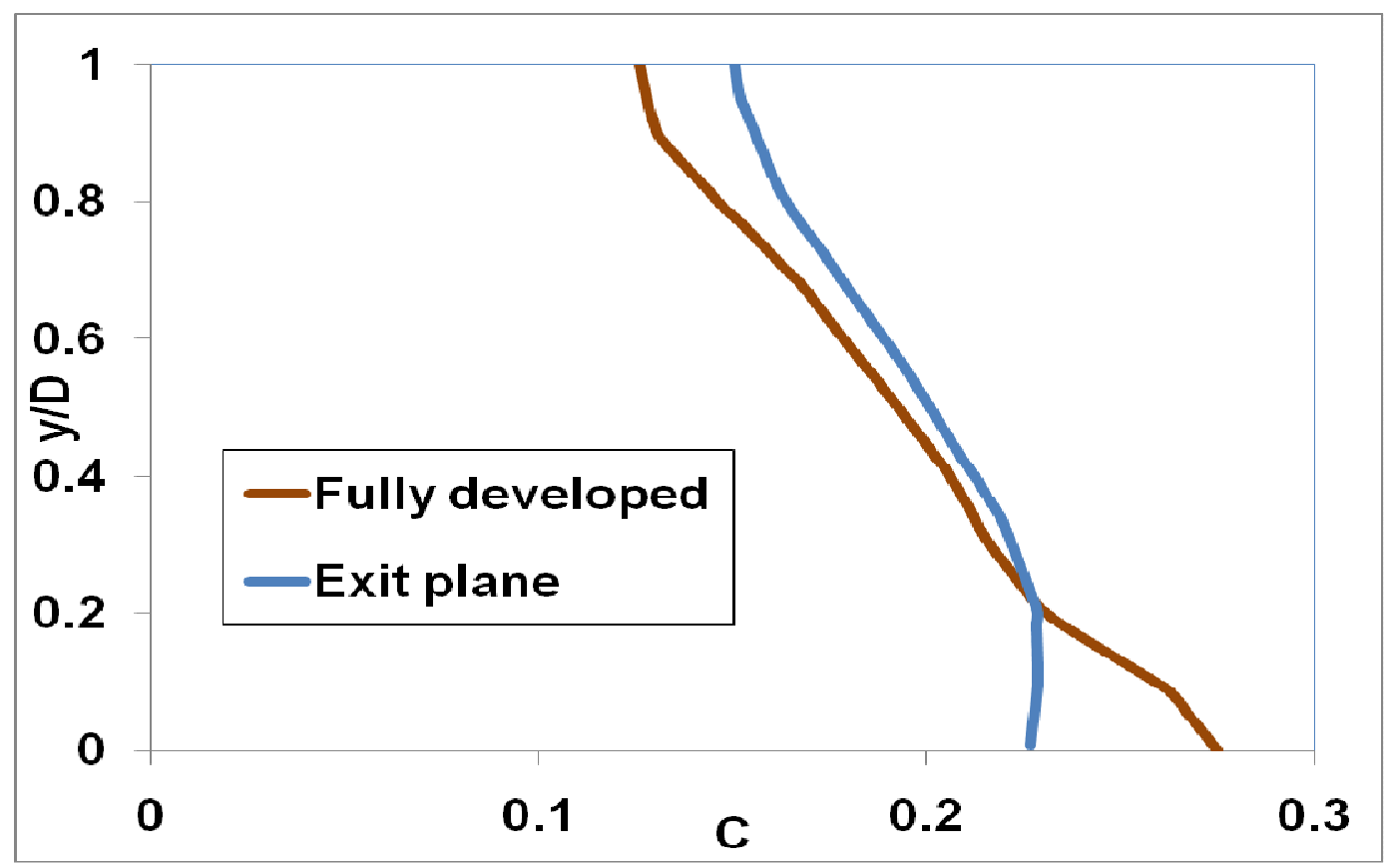

Figure 4.3.2.8: Concentration profiles of $150 \mu \mathrm{m}$ ADP particles at 0.2 initial efflux concentration as realized at the fully developed region and the exit plane 


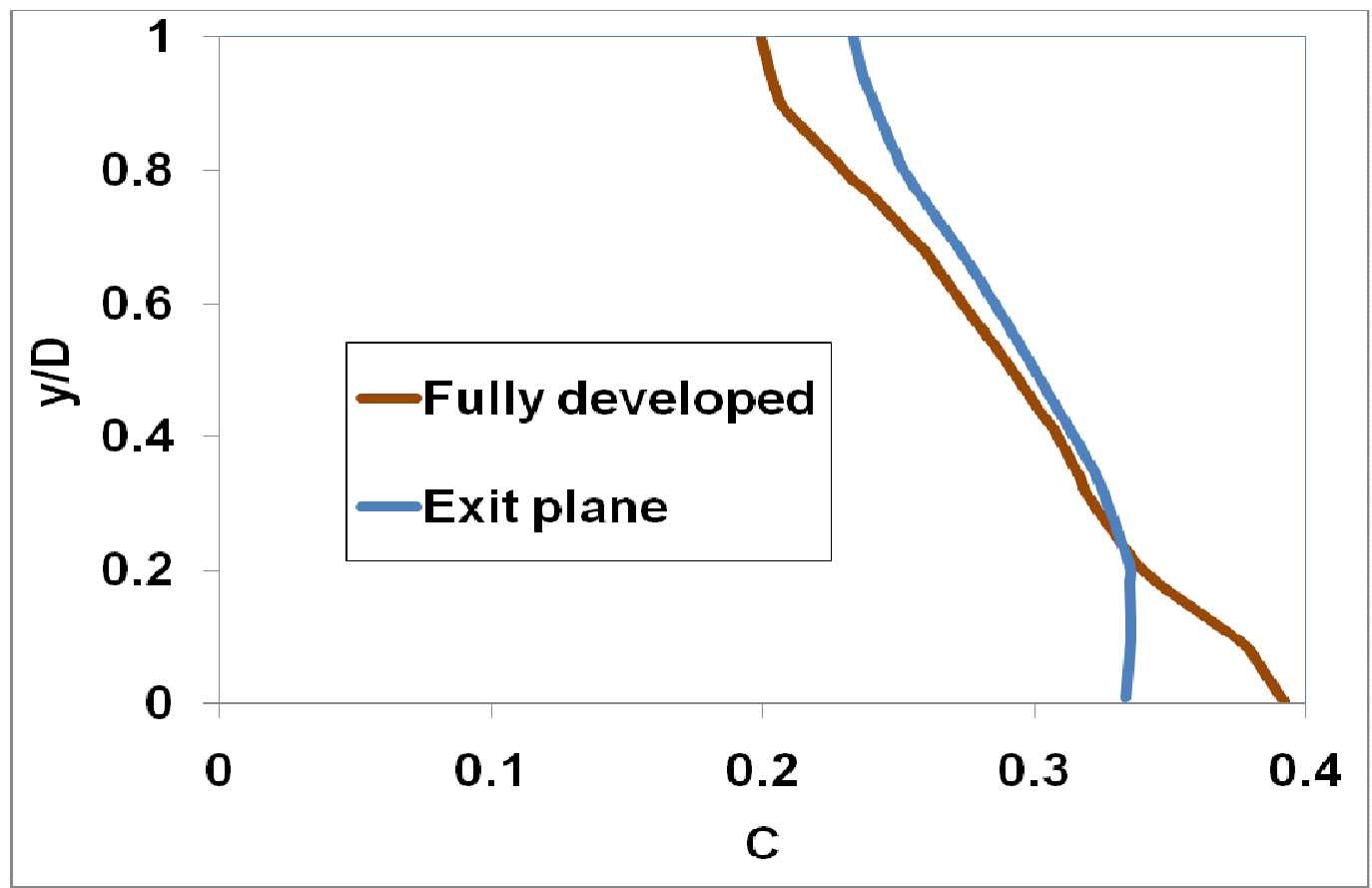

Figure 4.3.2.9: Concentration profiles of $150 \mu \mathrm{m}$ ADP particles at 0.3 initial efflux concentration as realized at the fully developed region and the exit plane

In all the above cases the concentration profiles at the exit plane display a certain degree of uniformity. This can be explained by considering the shear strain that is registered by the slurry when it travels through the rapidly accelerating section present in the pipeline. The strain rate is represented by Equation 4.3.2.1. As the slurry passes through the contraction, it rapidly accelerates. Its velocity shoots up from $2 \mathrm{~m} / \mathrm{s}$ to $8 \mathrm{~m} / \mathrm{s}$ in a short span of approximately $0.0254 \mathrm{~m}$ ( 1 inch). This causes a rapid rise in the shear strain along the axis of the geometry. The velocity vectors at the contracting section of the geometry are presented in Fig. 4.3.2.9. A two dimensional slice at the exact center of the volume is created, and contours of velocity are generated. These contours are presented in Fig 4.3.2.10. The behavior of shear strain along the axis of the nozzle is presented in Fig 4.3.2.11. It is worth noting that it was mentioned in Section 2.2, while describing rapid distortion theory that the strain rate increases to an enormous degree when the fluid passes through a symmetric geometric contraction. 


$$
\varepsilon_{x y}=\frac{1}{2} \frac{d \bar{U}}{d y}
$$

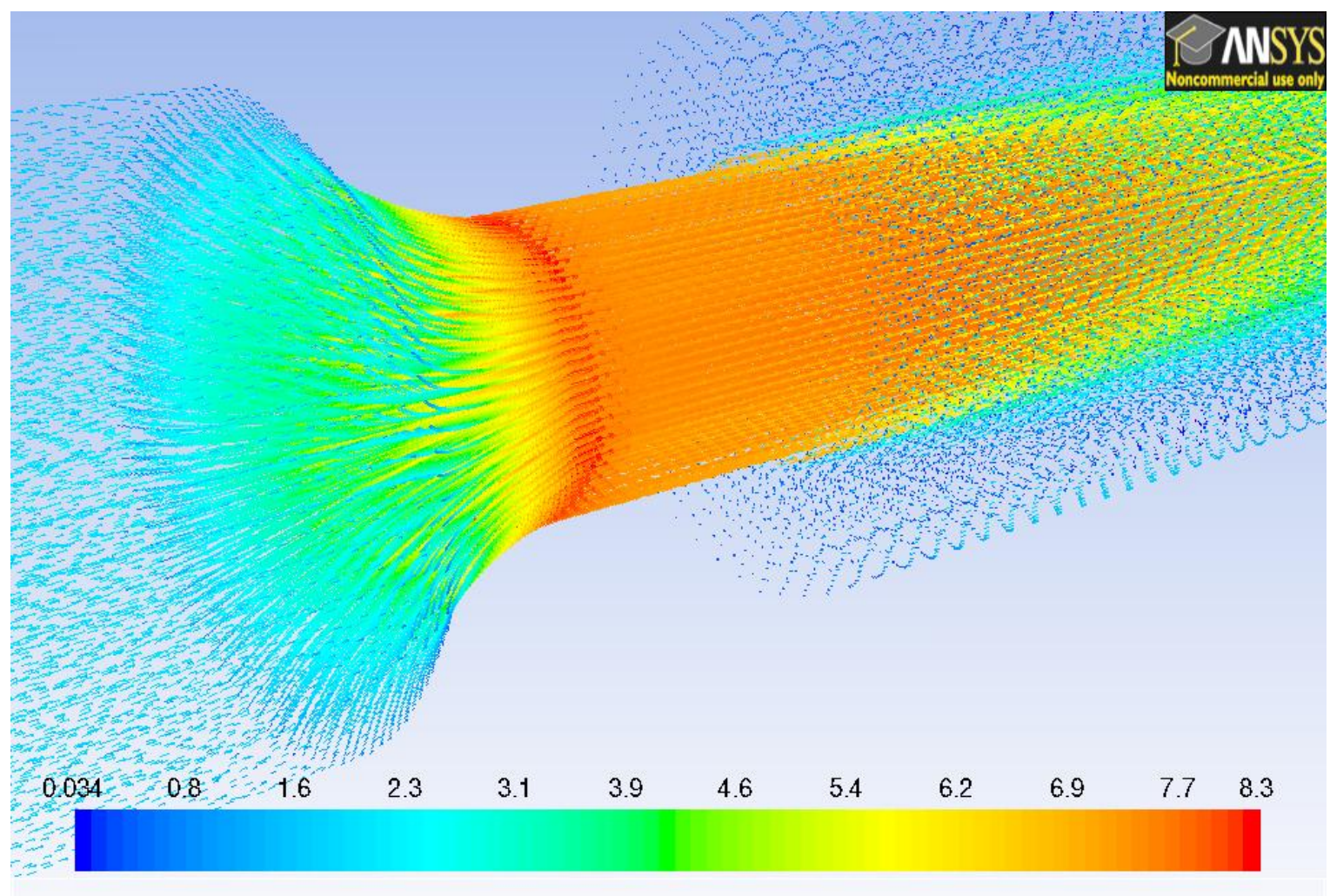

Velocity Vectors Colored By Velocity Magnitude (mixture) $(\mathrm{m} / \mathrm{s})$ (Time $=4.5000 e+00) \quad J u l 01,2010$ ANSYS FLUENT 12.0 (3d, dp, pbns, mixture, ske, transient)

Figure 4.3.2.10: Velocity vectors of a 5 percent ADP-xylene slurry $\left(d_{p}=38 \mu \mathrm{m}\right)$ passing through the ASME flow nozzle at Reynolds number $\left(\operatorname{Re}_{\mathrm{D}}\right)$ of 140000 


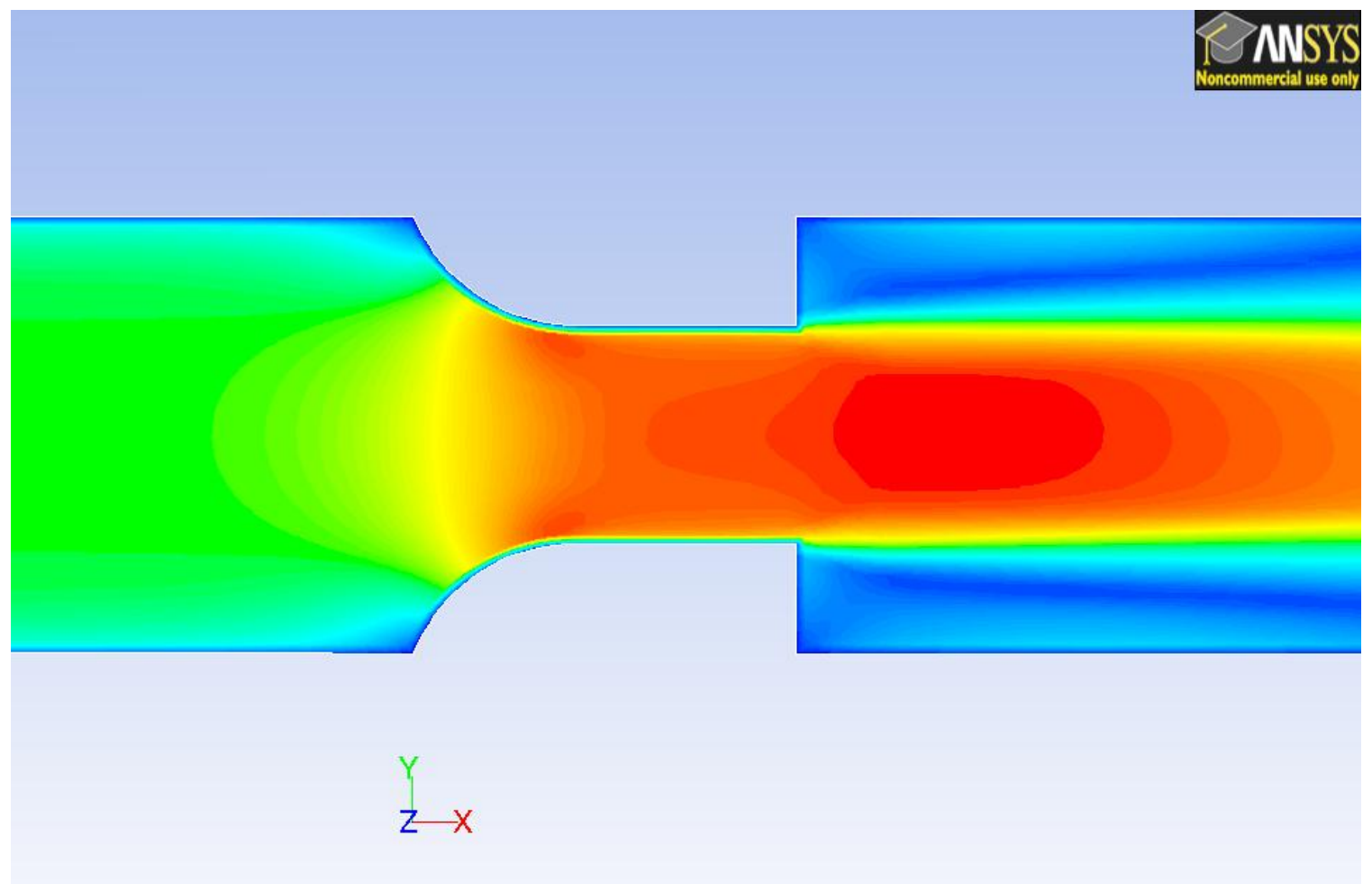

Figure 4.3.2.11: Velocity contours at the mid-plane of a 5 percent ADP-xylene slurry $\left(d_{p}=38\right.$ $\mu \mathrm{m})$ passing through the ASME flow nozzle at a Reynolds number $\left(\operatorname{Re}_{\mathrm{D}}\right)$ of 140000

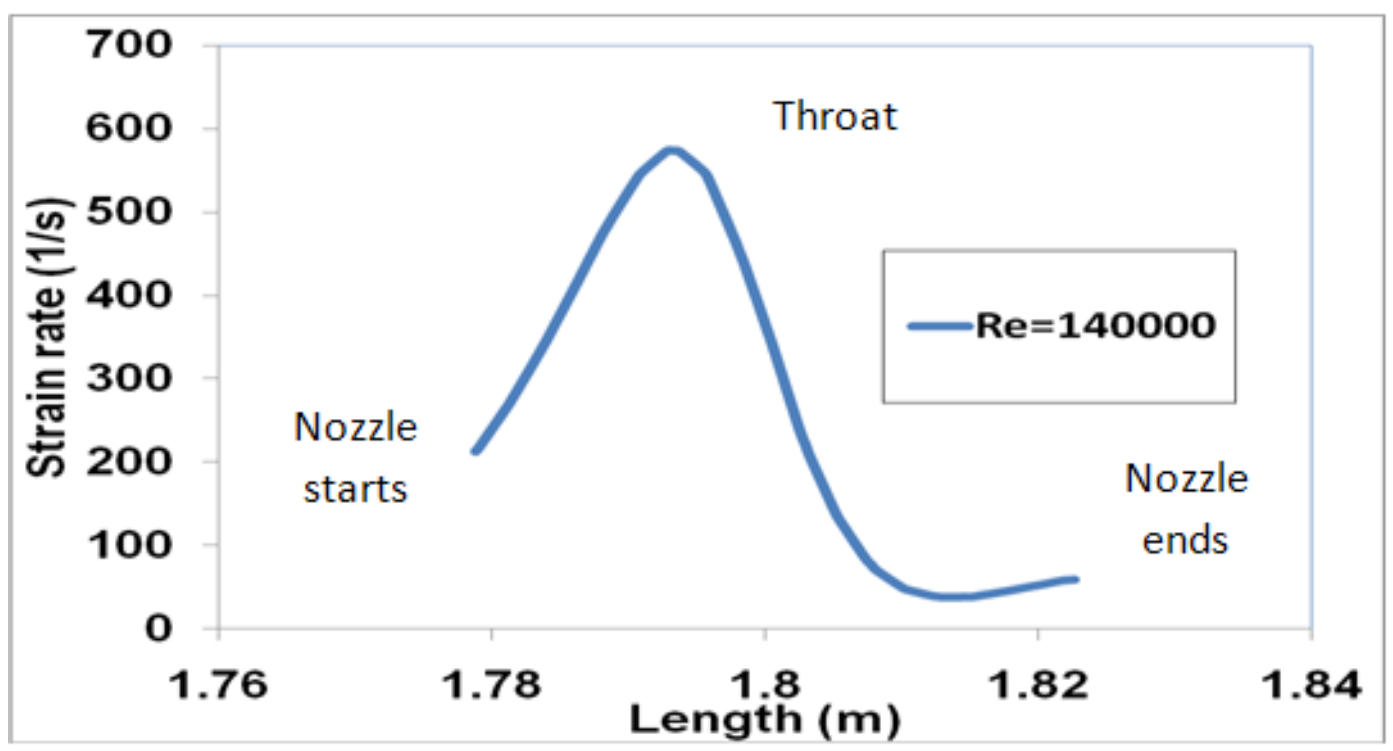

Figure 4.3.2.12: Strain rate registered by the 5 percent ADP-xylene slurry along the axis of the ASME nozzle at $\operatorname{Re}_{D}=140000$ 
4.3.3. Concentration profiles for $\left(\operatorname{Re}_{\mathrm{D}}=280000\right)$

The concentration profiles that belong to the cases pertaining to Reynolds number $\left(\operatorname{Re}_{\mathrm{D}}=280000\right)$ will be presented and discussed in this section. Since the Reynolds number of the flow has increased, the fully-developed region shifts to a location that is further downstream. The distance of this location from the entrance can be estimated by using Equation 4.4.3.1 (Pope 2000).

$$
E l_{\text {turbulent }}=4.4 \operatorname{Re}_{D}{ }^{1 / 6}
$$

Using the above equation, it was estimated that the fully developed flow condition is achieved at $\mathrm{X} / \mathrm{D} 34$. The location of the fully developed region with respect to the exit plane of the nozzle is presented in Fig 4.3.3.1.

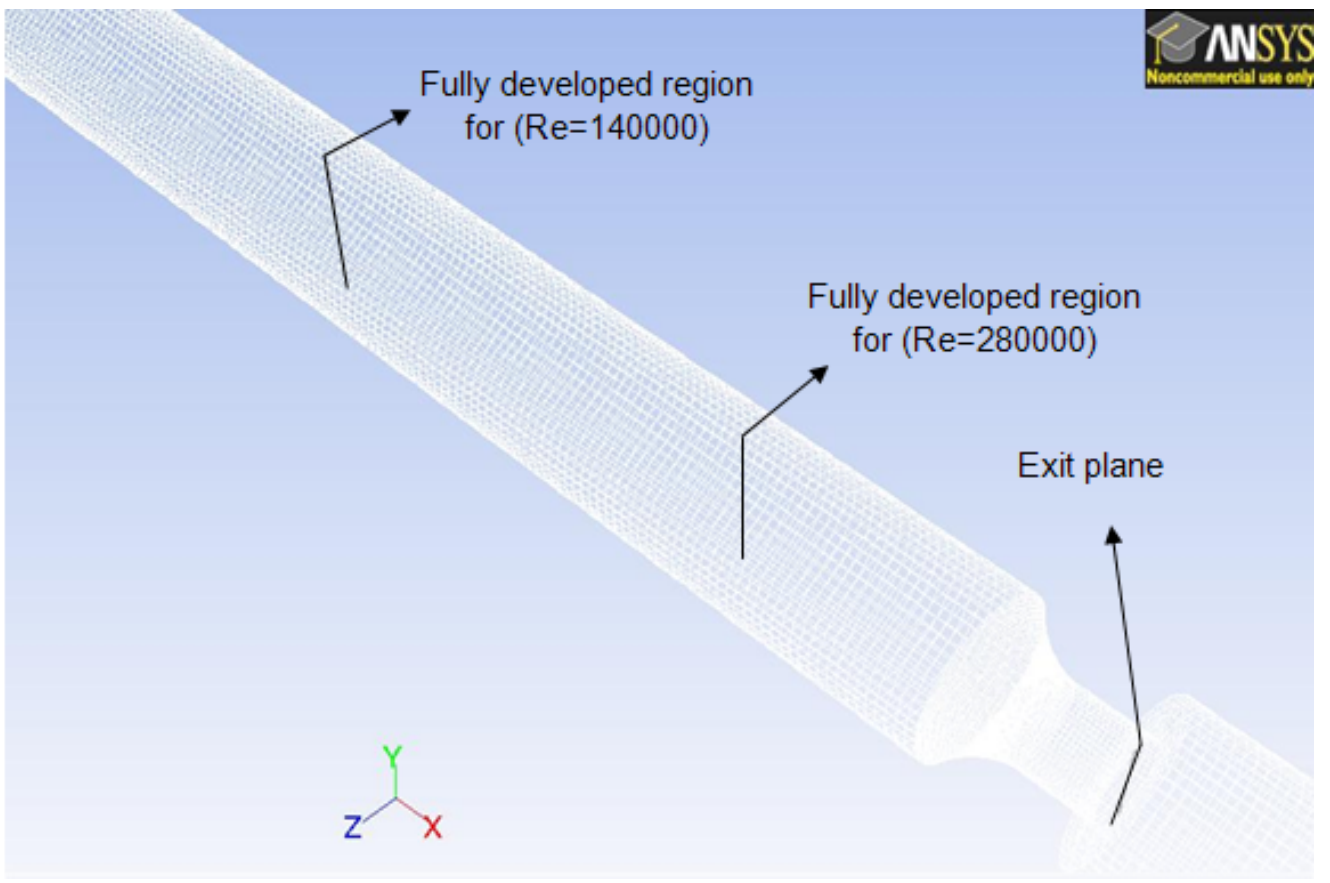

Figure 4.3.3.1: Position of fully developed region with respect to the exit plane of the nozzle (Represented by dark vertical lines) 
The concentration profiles at the fully developed region and the exit plane of the nozzle are plotted on the same area to enhance comparison. The graphs appear in the increasing order of particle size and initial efflux concentration. A brief discussion after each set of plots throws more light on what is happening at the fully developed region and the exit plane of the geometry.

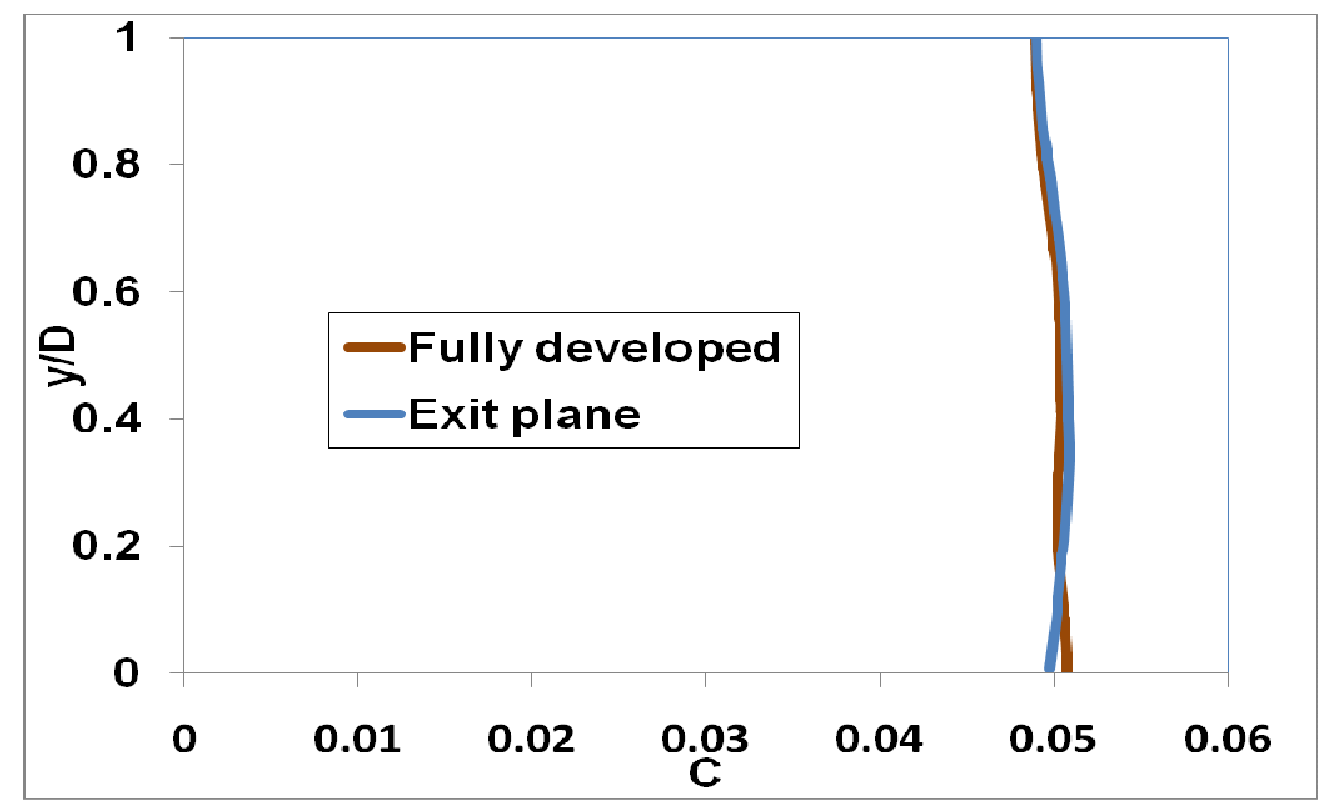

Figure 4.3.3.2: Concentration profiles of $38 \mu \mathrm{m}$ ADP particles at 0.05 initial efflux concentration as realized at the fully developed region and the exit plane 


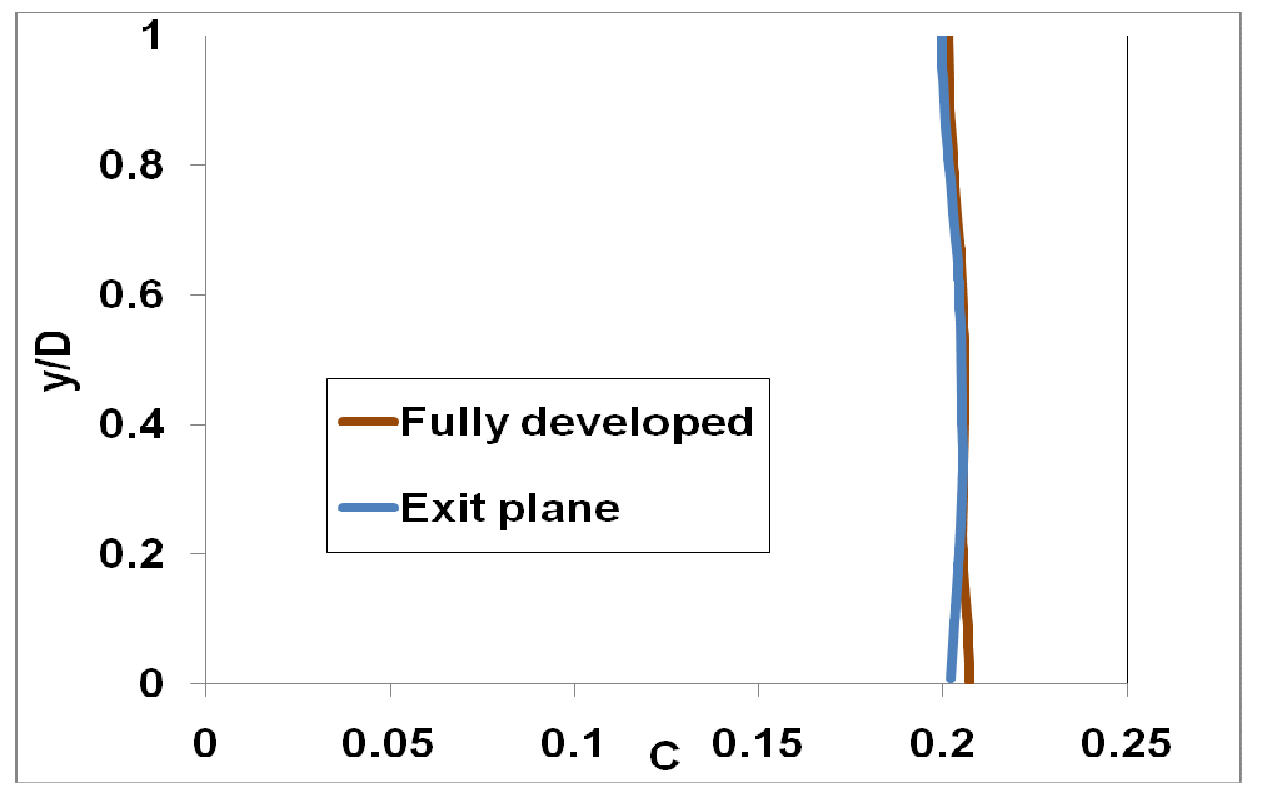

Figure 4.3.3.3: Concentration profiles of $38 \mu \mathrm{m}$ ADP particles at 0.2 initial efflux concentration as realized at the fully developed region and the exit plane

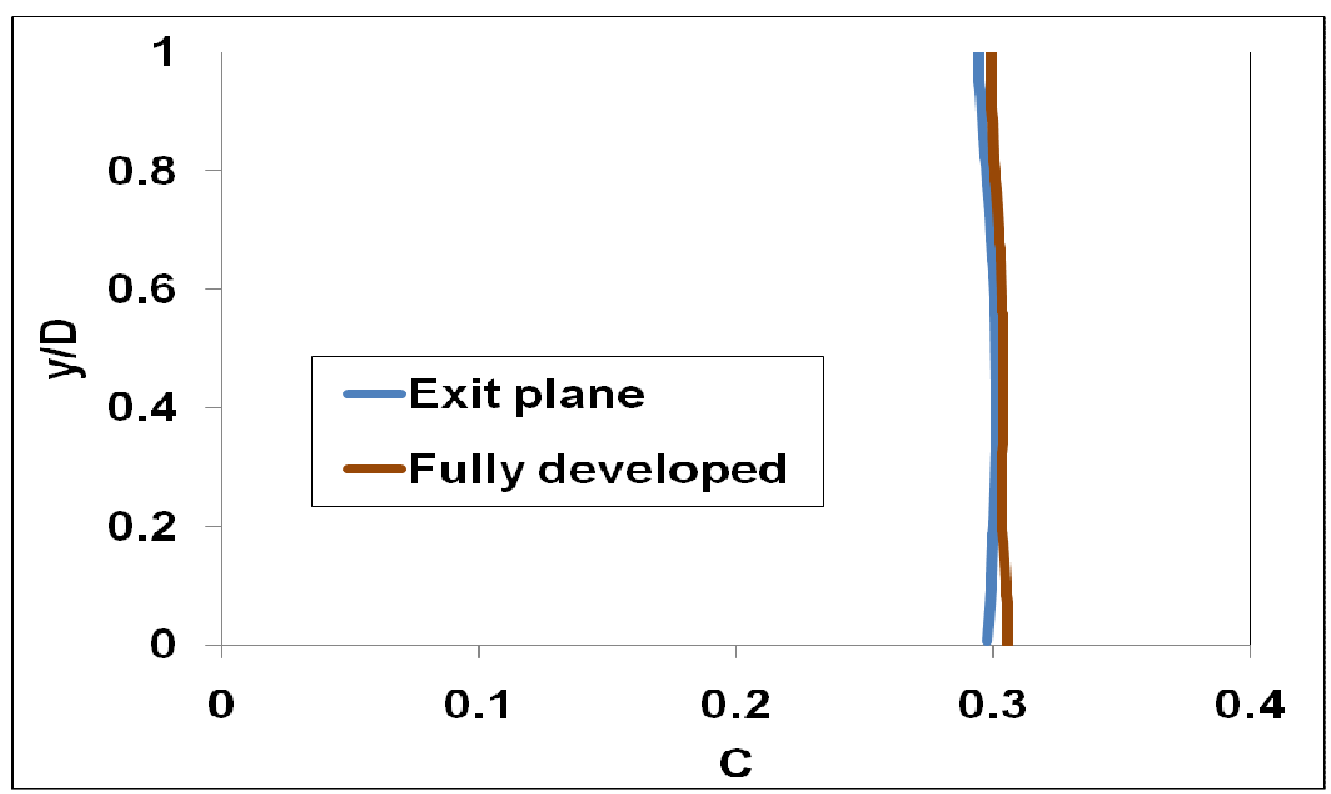

Figure 4.3.3.4: Concentration profiles of $38 \mu \mathrm{m}$ ADP particles at 0.3 initial efflux concentration as realized at the fully developed region and the exit plane

After observing Fig 4.3.3.2 through 4.3.3.4, one finds that the concentration profiles registered at the fully developed region and the exit plane at any initial efflux concentration are very similar. Since the Reynolds number $\left(\operatorname{Re}_{\mathrm{D}}\right)$ has increased to 280000 , the $38 \mu \mathrm{m}$ particles are acted upon by greater turbulent eddy drag force. This turbulent eddy drag force, the primary agent that keeps the 
particles suspended in a two-phase flow, substantially compensates for the gravity force, thus maintaining a nearly uniform concentration of particles throughout the cross-section of the pipe geometry. However, a closer observation of the figures reveals that the concentration profile realized at the exit plane is yet again closer to the initial efflux concentration. How do the concentration profiles of heavier particles $(75 \mu \mathrm{m})$ behave at a higher Reynolds number $\left(\operatorname{Re}_{D}=280000\right)$ can be understood by observing the figures that follow.

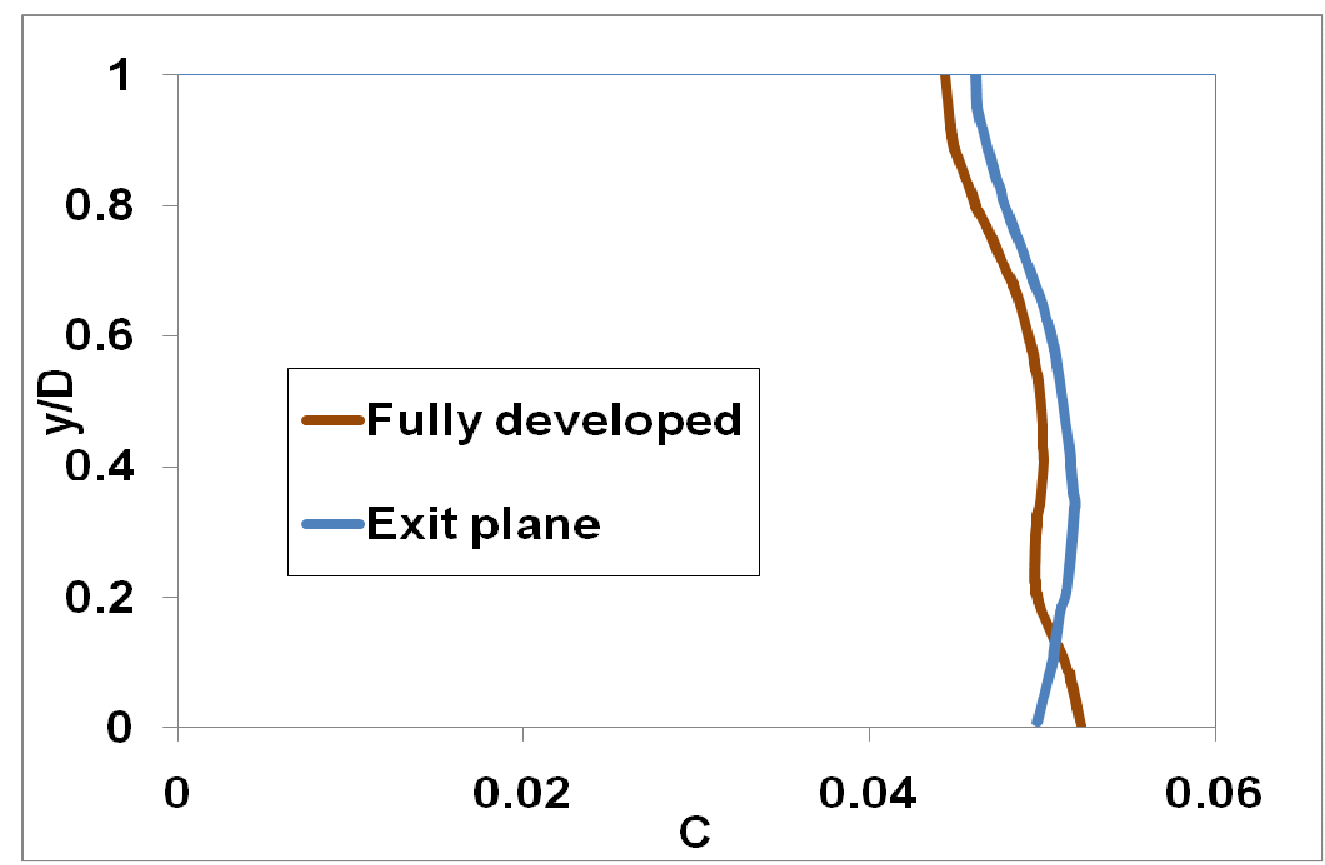

Figure 4.3.3.5: Concentration profiles of $75 \mu \mathrm{m}$ ADP particles at 0.05 initial efflux concentration as realized at the fully developed region and the exit plane 


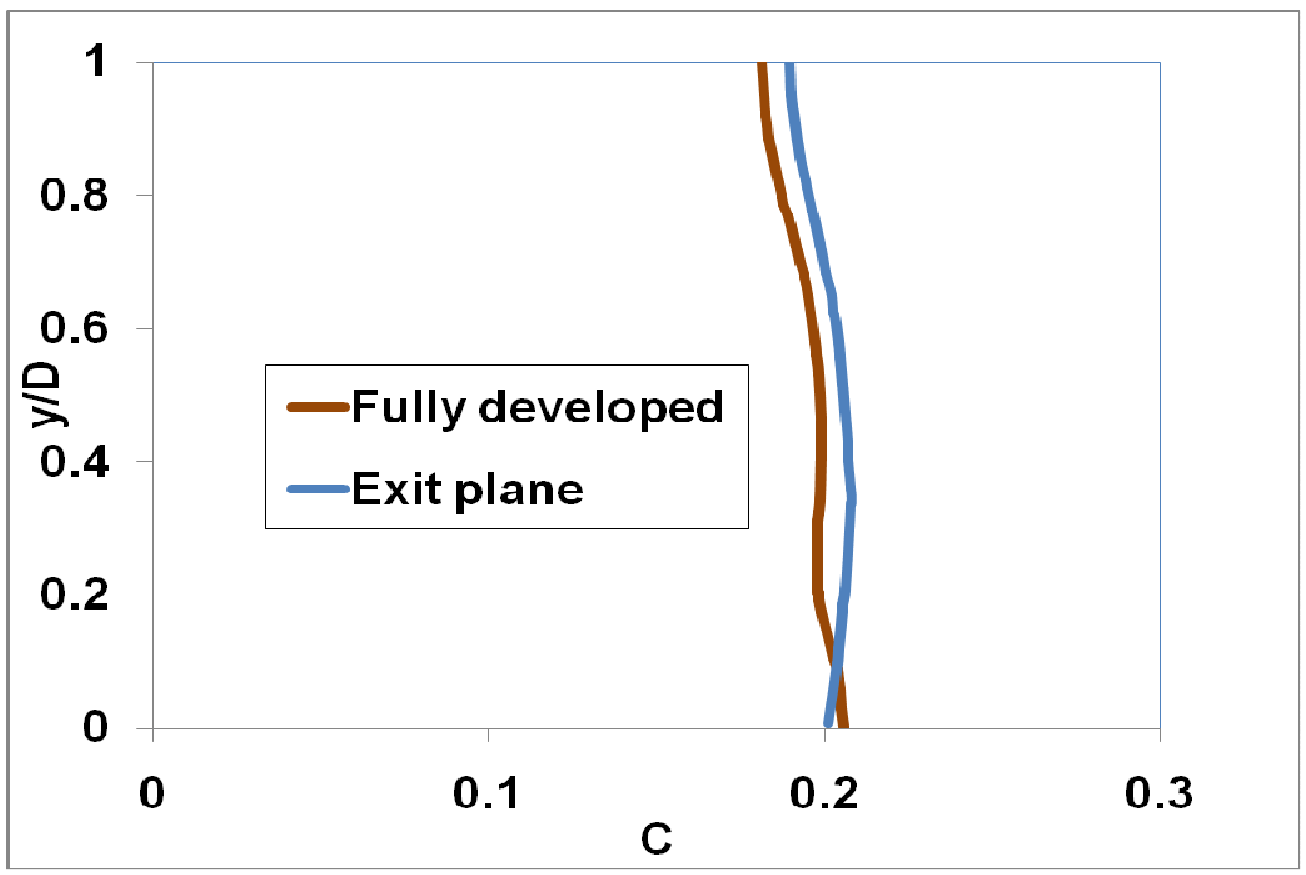

Figure 4.3.3.6: Concentration profiles of $75 \mu \mathrm{m}$ ADP particles at 0.2 initial efflux concentration as realized at the fully developed region and the exit plane

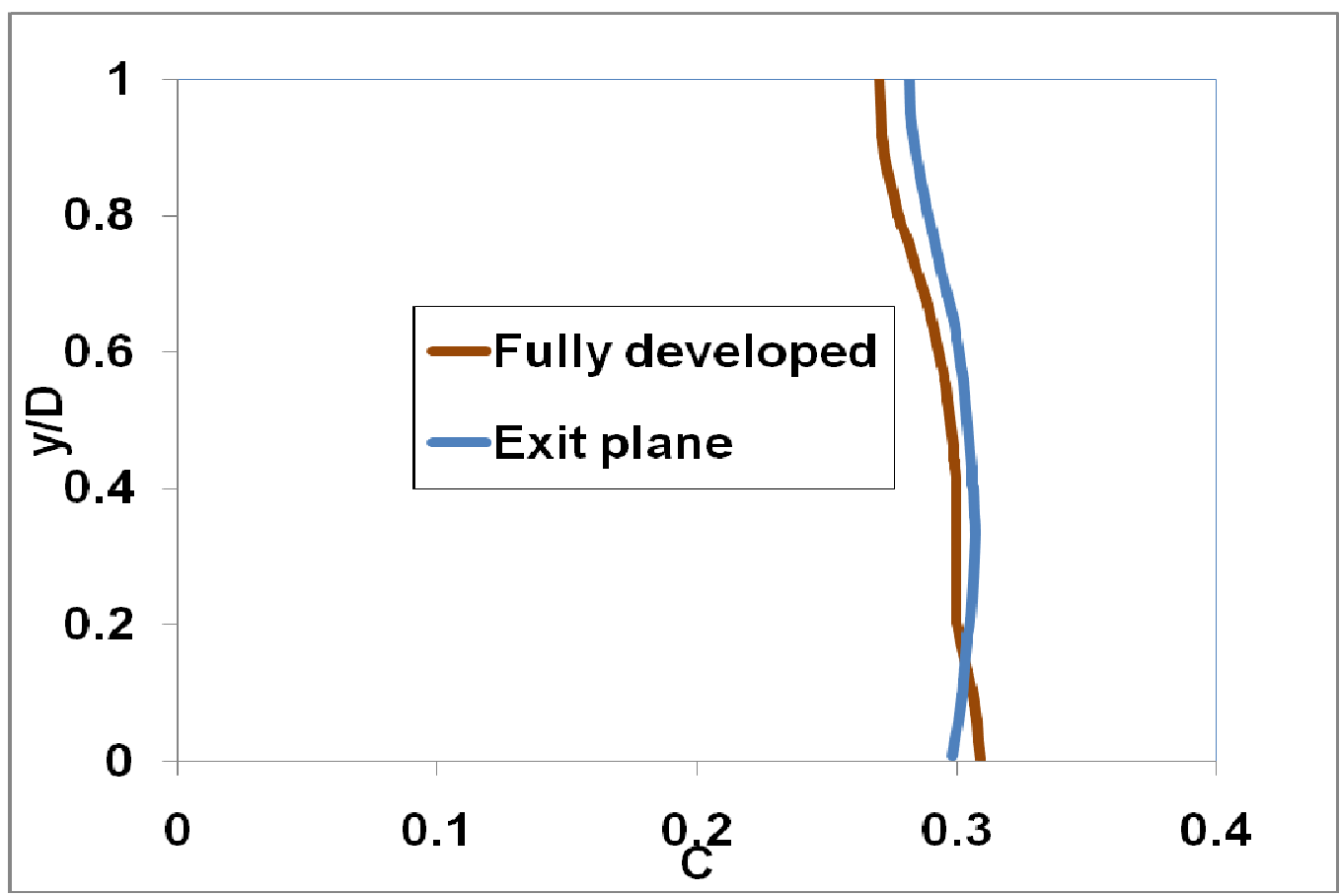

Figure 4.3.3.7: Concentration profiles of $75 \mu \mathrm{m}$ ADP particles at 0.3 initial efflux concentration as realized at the fully developed region and the exit plane 
One of the most likely observations that one would make after going through the above graphs is that there is a subtle "bump like" enlargement at the core region of the pipe flow. This was almost imperceivable in all the low Reynolds number cases $\left(\operatorname{Re}_{\mathrm{D}}=140000\right)$. The aforementioned peculiarity can also be observed in the results of Roco and Shook (1984), presented in Fig 2.1.4. Roco and Shook worked with sand-water slurries where the Reynolds number of the flow was in the same order of magnitude of the current case. Moreover, after observing the profiles at the exit plane of the nozzle, one would certainly feel that this effect intensifies at this location. Whether the increase in the Reynolds number has a pronounced effect on the heavier particles is what will be presented next. The following figures are for the $150 \mu \mathrm{m}$ particles.

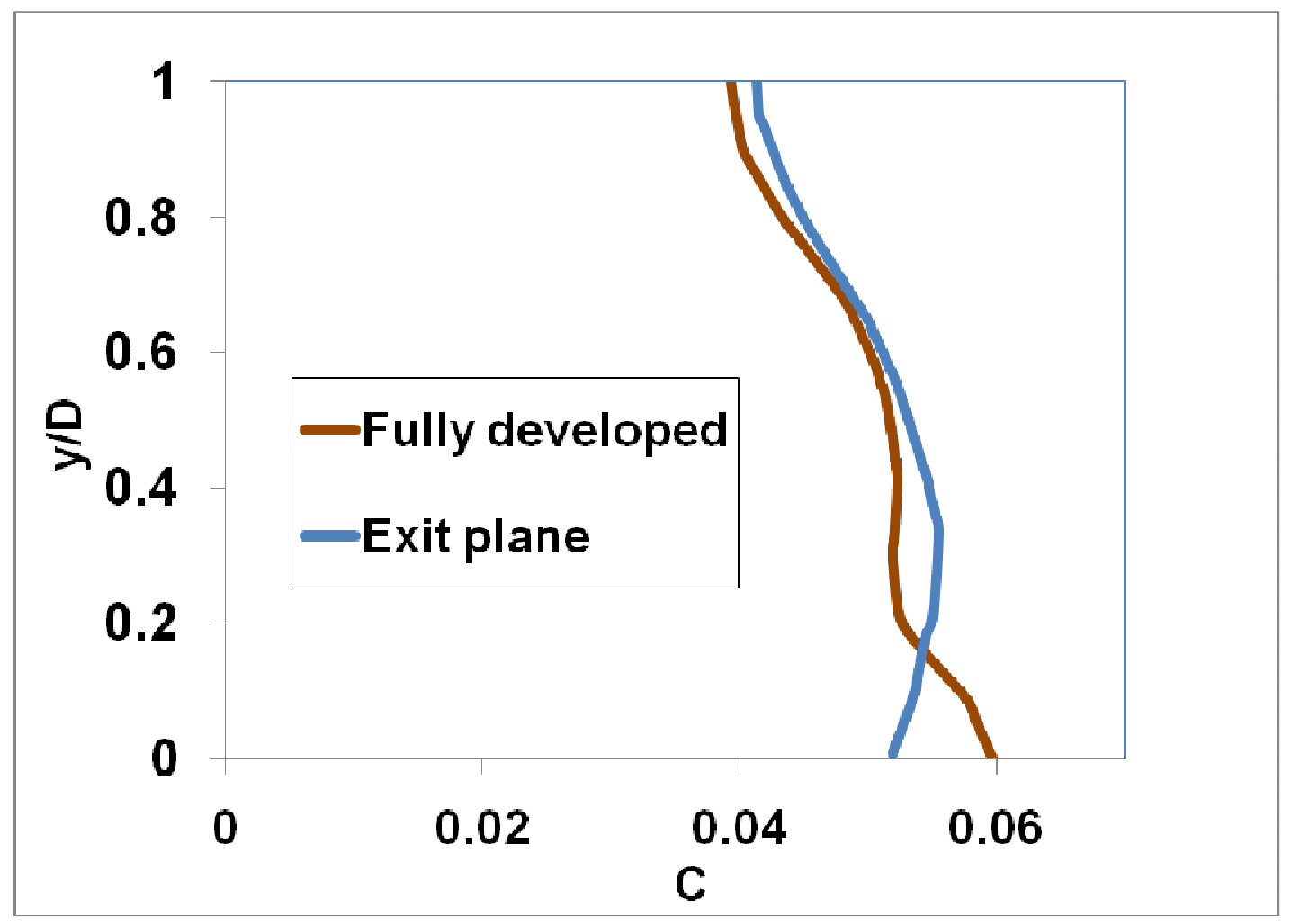

Figure 4.3.3.8: Concentration profiles of $150 \mu \mathrm{m}$ ADP particles at 0.05 initial efflux concentration as realized at the fully developed region and the exit plane 


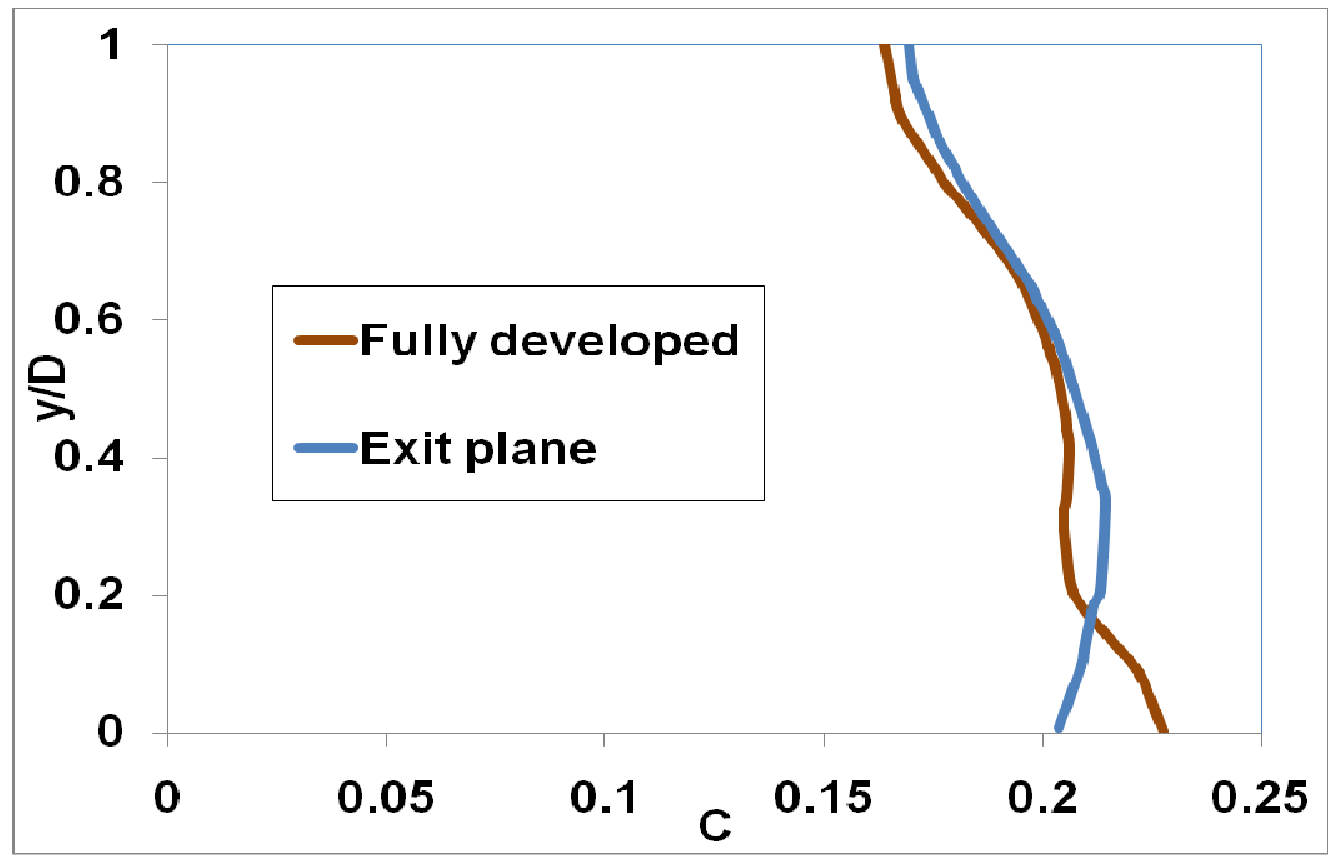

Figure 4.3.3.9: Concentration profiles of $150 \mu \mathrm{m}$ ADP particles at 0.2 initial efflux concentration as realized at the fully developed region and the exit plane

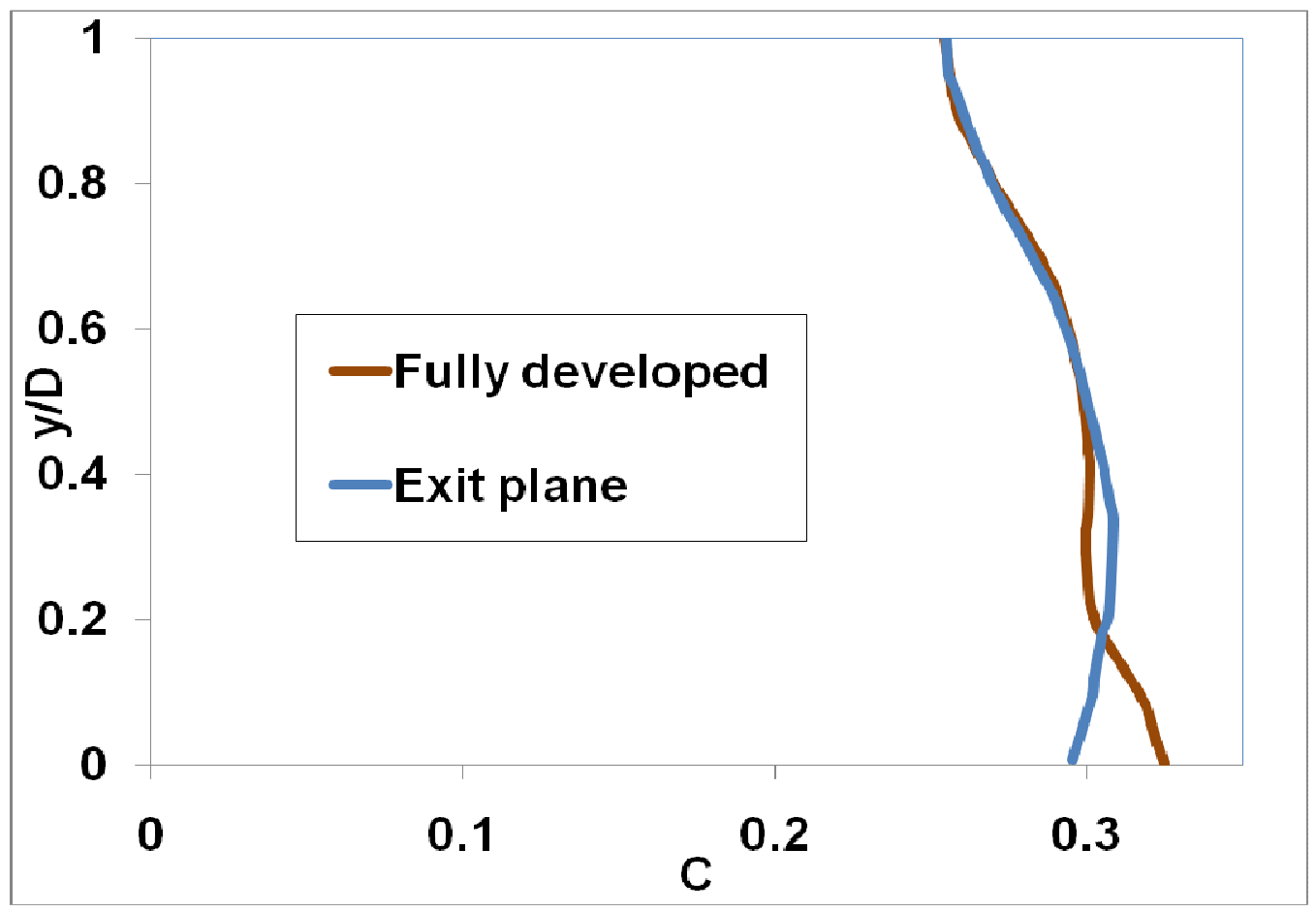

Figure 4.3.3.10: Concentration profiles of $150 \mu \mathrm{m}$ ADP particles at 0.3 initial efflux concentration as realized at the fully developed region and the exit plane 
After studying Fig 4.3.3.7 through Fig 4.3.3.9, it seems that the geometric contraction has a bigger effect on the particle concentration profiles of heavier particles. A plausible explanation for the shift in the concentration profile towards the initial efflux concentration at the exit plane of the nozzle is presented in the section that follows.

\subsection{Discussion}

A good number of cases varying the particle size, initial efflux concentration, and the Reynolds number were presented in the previous section. These cases confirm that the concentration profiles at the exit plane of a symmetric contraction (ASME flow nozzle) are more uniform when compared with those at the fully developed region, and are shifted closer to the initial efflux concentration. In the small particle size $\left(d_{p}=38 \mu \mathrm{m}\right)$ and low Reynolds number $\left(\mathrm{Re}_{\mathrm{D}}=140000\right)$ case, it can be observed that the concentration profiles at the exit plane remain comparatively uniform when compared with those at the fully developed region. This section explains this effect by employing the theory of rapid distortion.

It was mentioned earlier that as the flow passes through a symmetric geometric contraction, the mean strain rate in the direction of the main flow is so large and the turbulence is so weak that the usual turbulent exchange mechanisms are rendered passive (Libby 1996). This is the essence of the rapid distortion theory. It has already been shown in Fig 4.3.2.11 that the strain rate increases to an enormous degree along the axis of the geometric contraction. A comparison between the strain rates at the two Reynolds numbers $\left(\operatorname{Re}_{\mathrm{D}}\right)$ that were employed is presented in this section.

The most suitable way to quantify 'weak turbulence' is to compute and plot the local turbulence intensity $(I)$. The turbulence intensity $(I)$ is presented in Equation 4.4.1. 


$$
I=\frac{\sqrt{\frac{2 k}{3}}}{\bar{U}}
$$

Where,

$k=$ Turbulence kinetic energy, $\left(\mathrm{m}^{2} / \mathrm{s}^{2}\right)$

$\bar{U} \quad=\quad$ Reynolds averaged centre line mean fluid velocity, $(\mathrm{m} / \mathrm{s})$

Numerous attempts to obtain a steady state solution for a two-phase flow (ADP-xylene) failed. Perhaps, it was too ambitious of me to expect a steady state solution for a multi-phase flow to reach convergence, despite being aware of the fact that it is highly recommended to opt for a transient formulation in case of multi-phase flows owing to particle-particle collisions. However, it is safe to assume that the secondary phase (ADP particles) will follow the primary phase as the Stokes number in all the cases is less than unity, see Equation 3.4.1. Hence a steady state solution for just the primary phase was simulated.

Initially, the behavior of the mean strain rate along the axis of the flow nozzle is presented in Fig 4.4.1. Later, the turbulent intensity (I) realized at the fully developed region and the exit plane of the nozzle is plotted on the same graph to enhance comparison. 


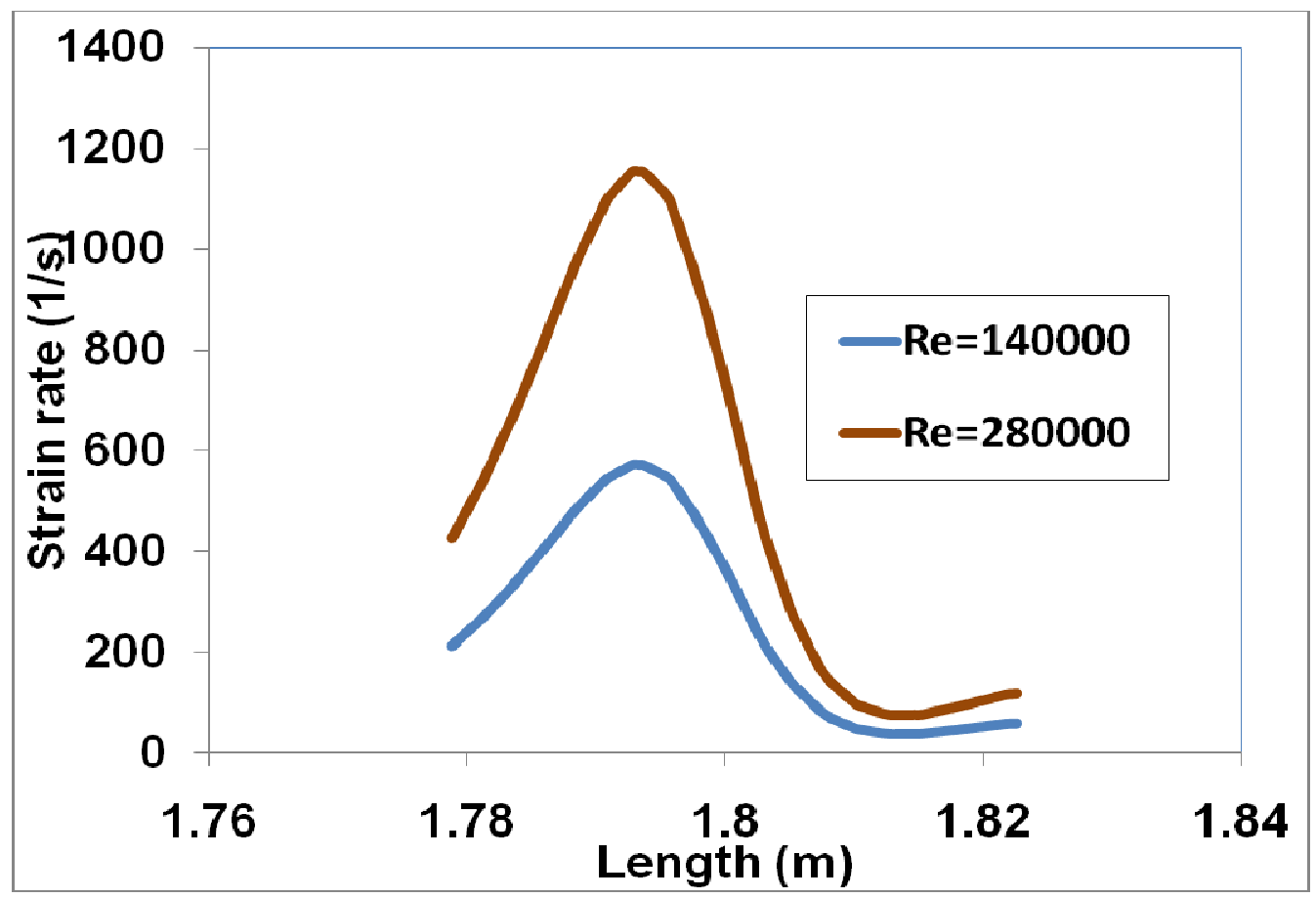

Figure 4.4.1: Comparison of mean strain rates along the axis of the ASME flow nozzle at two Reynolds numbers $\left(\operatorname{Re}_{\mathrm{D}}\right)$

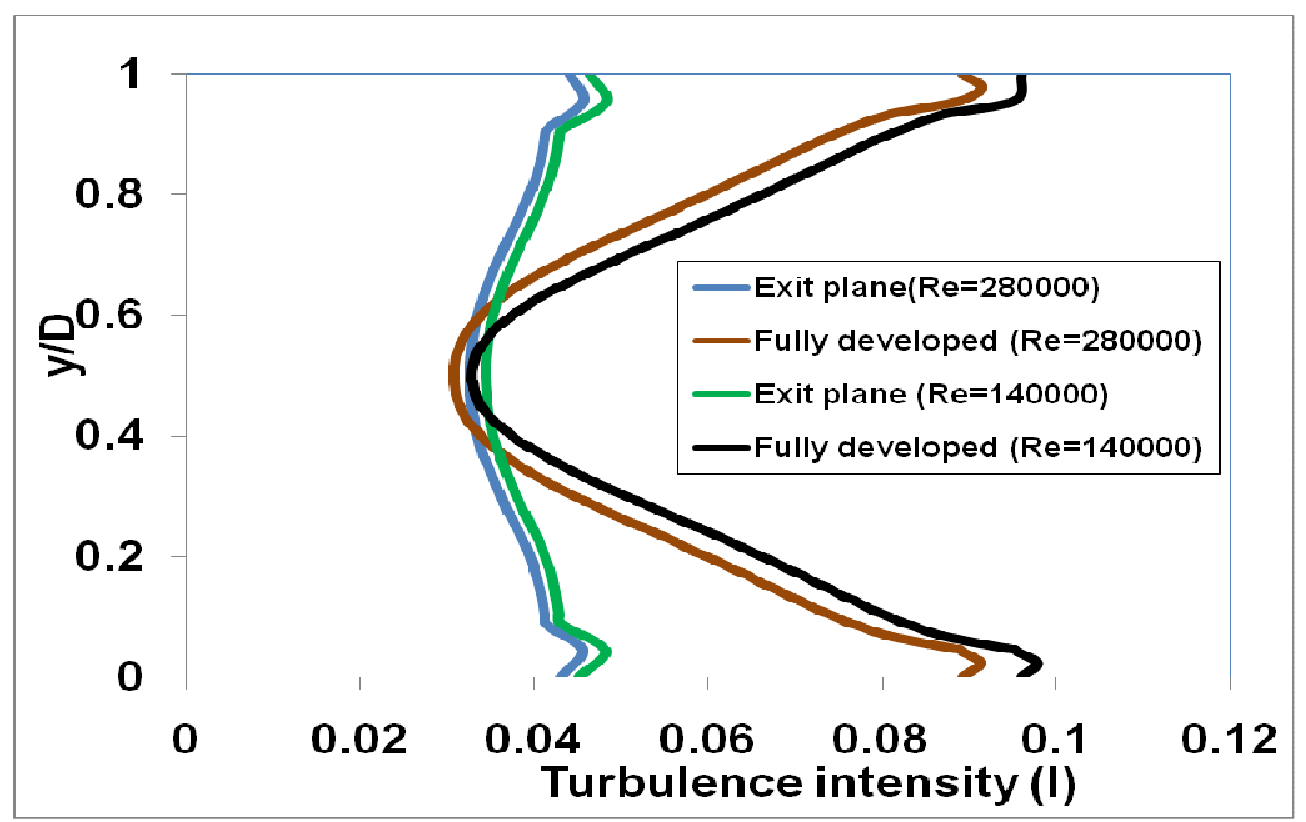

Figure 4.4.2: Comparison of turbulence intensities (I) at the fully developed region and the exit plane 
There are two observations that one can make from Fig 4.4.2. The more trivial of the two is that the turbulence intensities for the two Reynolds numbers $\left(\mathrm{Re}_{\mathrm{D}}\right)$ do not vary by a great margin. This can be explained by Equation 4.4.2.

$$
I=0.16\left(\operatorname{Re}_{D}\right)^{-1 / 8}
$$

When one substitutes the two aforementioned Reynolds numbers in Equation 4.4.2, they will find that the turbulence intensity (I) values differ by a very small magnitude.

The observation that holds greater importance is that the turbulence intensity (I) at the exit plane of the ASME flow nozzle is lower when compared to that realized at the fully developed region. Further, one can also notice large reduction in turbulence intensity close to the wall. This happens in both the low Reynolds number case and the high Reynolds number case. The contours of turbulence intensity (I) at the mid-plane are presented in Fig 4.4.4. The velocity profiles of xylene at the fully developed region and the exit plane are presented in Fig 4.4.5 and Fig 4.4.6.

It has to be noted that the k-epsilon turbulence model employed in this study incorporates the Boussinesq eddy viscosity approximation. As mentioned previously, the eddy viscosity approximation helps one compute the Reynolds stress tensor as the product of an eddy viscosity and the mean strain rate tensor. In addition, the eddy viscosity approximation provides a most convenient closure to the Reynolds stress equations. Thus, for many flows the accuracy of this approximation is modest (Pope 2000). Since the Reynolds stress models solve the partial differential equations to compute the Reynolds stress tensor, their accuracy is greater when compared to the turbulence models that employ the eddy viscosity approximation. Explicitly, the Reynolds stress model predicts all the three components of Reynolds stresses more accurately than the models that employ Boussinesq approximation. 
As the turbulent flow through the symmetric contraction is realized, the root-mean-square velocity fluctuations in the $\mathrm{Y}$ and $\mathrm{Z}$ directions increase at the same rate, while the fluctuations in the $\mathrm{X}$ direction decrease. This causes the production of Reynolds stress in the $\mathrm{Y}$ and $\mathrm{Z}$ directions to increase, while the production of the same quantity in the $\mathrm{X}$ direction decreases almost linearly.

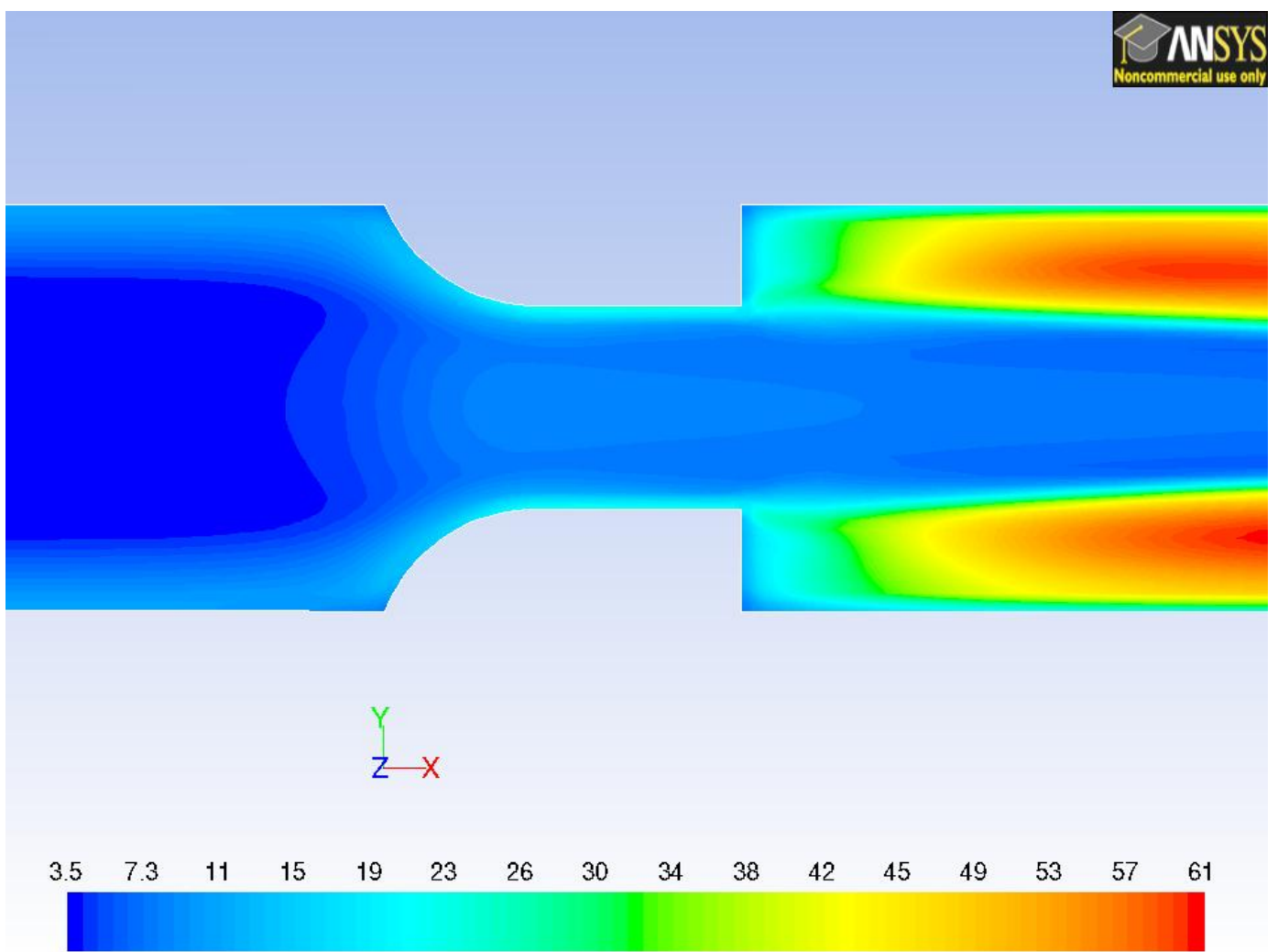

Figure 4.4.3: Contours of turbulence intensity at the centre plane of the control volume 


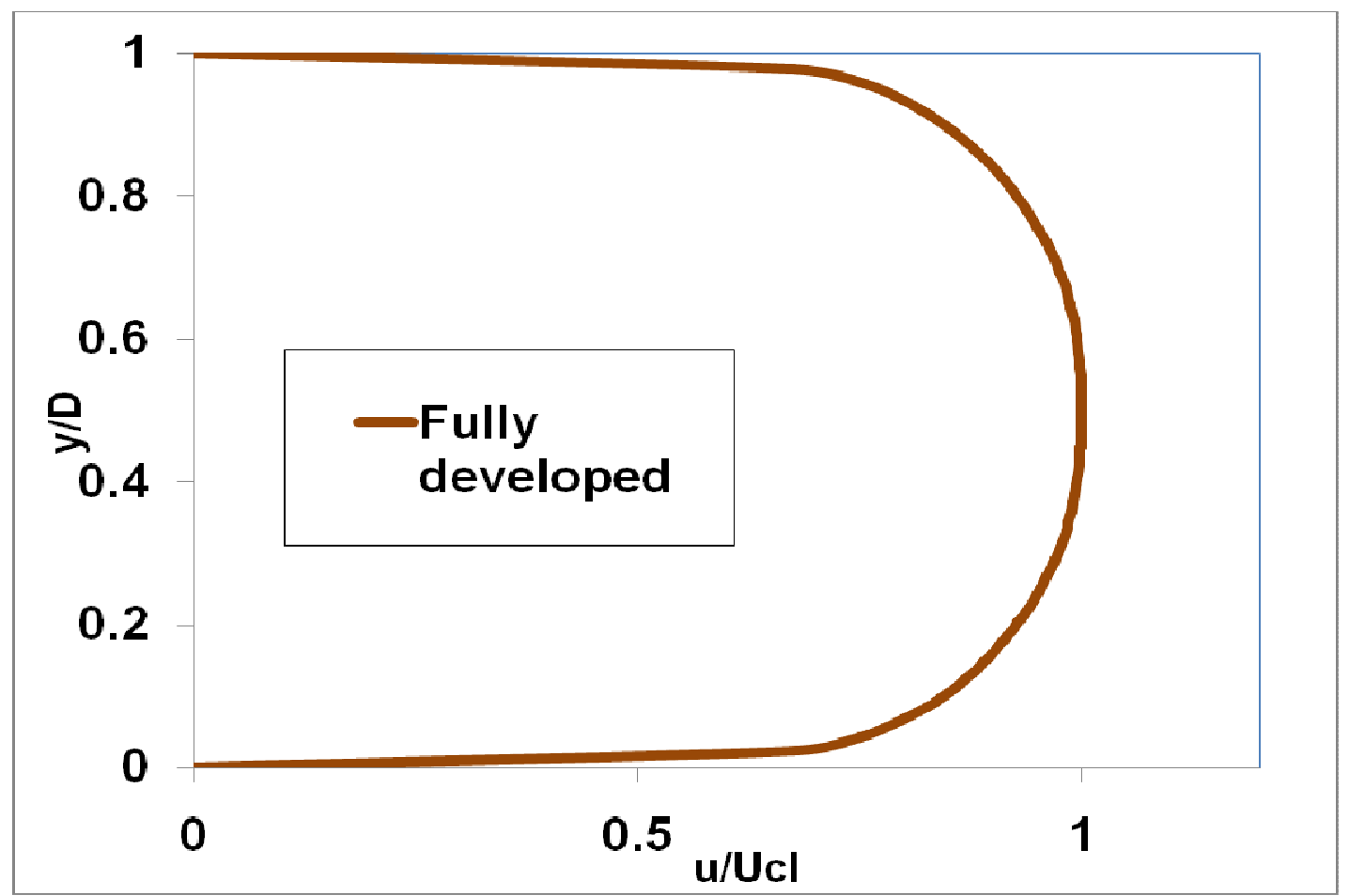

Figure 4.4.4: Fully developed velocity profile of xylene at a Reynolds number $\left(\operatorname{Re}_{\mathrm{D}}\right)$ of 140000

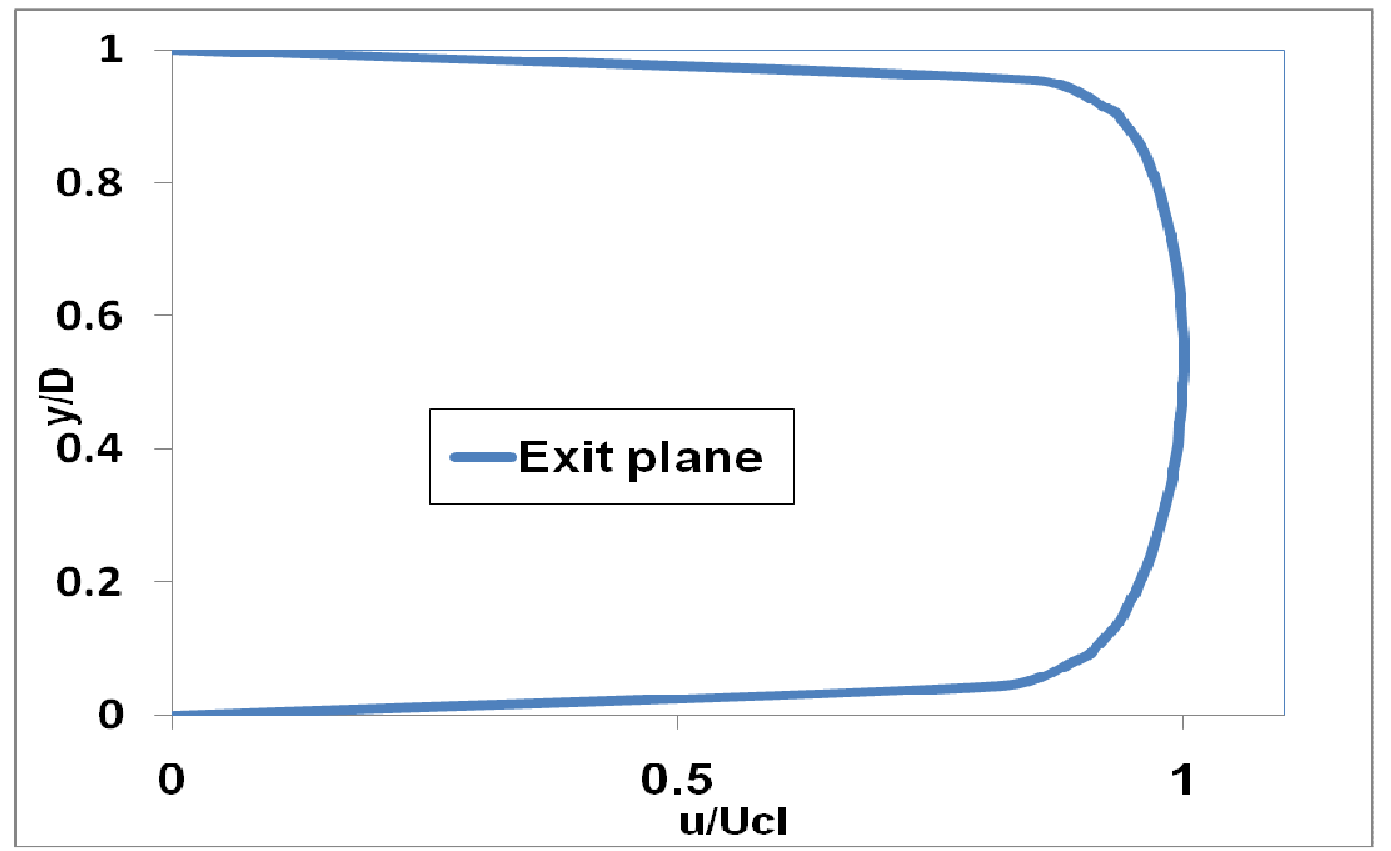

Figure 4.4.5: Velocity profile of xylene at the exit plane of the ASME flow nozzle at a Reynolds number $\left(\operatorname{Re}_{\mathrm{D}}\right)$ of 140000 
One can clearly observe that the velocity profile registered at the exit plane of the ASME flow nozzle is more uniform when compared to that realized at the fully developed region.

The effect of a geometric contraction on the concentration profiles was graphically represented in Section 4.2 and Section 4.3. The following sections make an effort to represent this effect visually.

\subsubsection{Contours of volume fraction $\left(\operatorname{Re}_{\mathrm{D}}=140000\right)$}

This section presents the contours of volume fraction of the secondary-phase (ADP particles) present in the slurry. The contours will be presented in the increasing order of particle size. All the contours are for an initial efflux volumetric concentration of 0.30 . Cross-section contours of the fully developed region and the exit plane will also be presented for $150 \mu \mathrm{m}$ particles. The direction of gravity in all the figures presented in Sections 4.4.1 and 4.4.2 is in the negative Y direction.

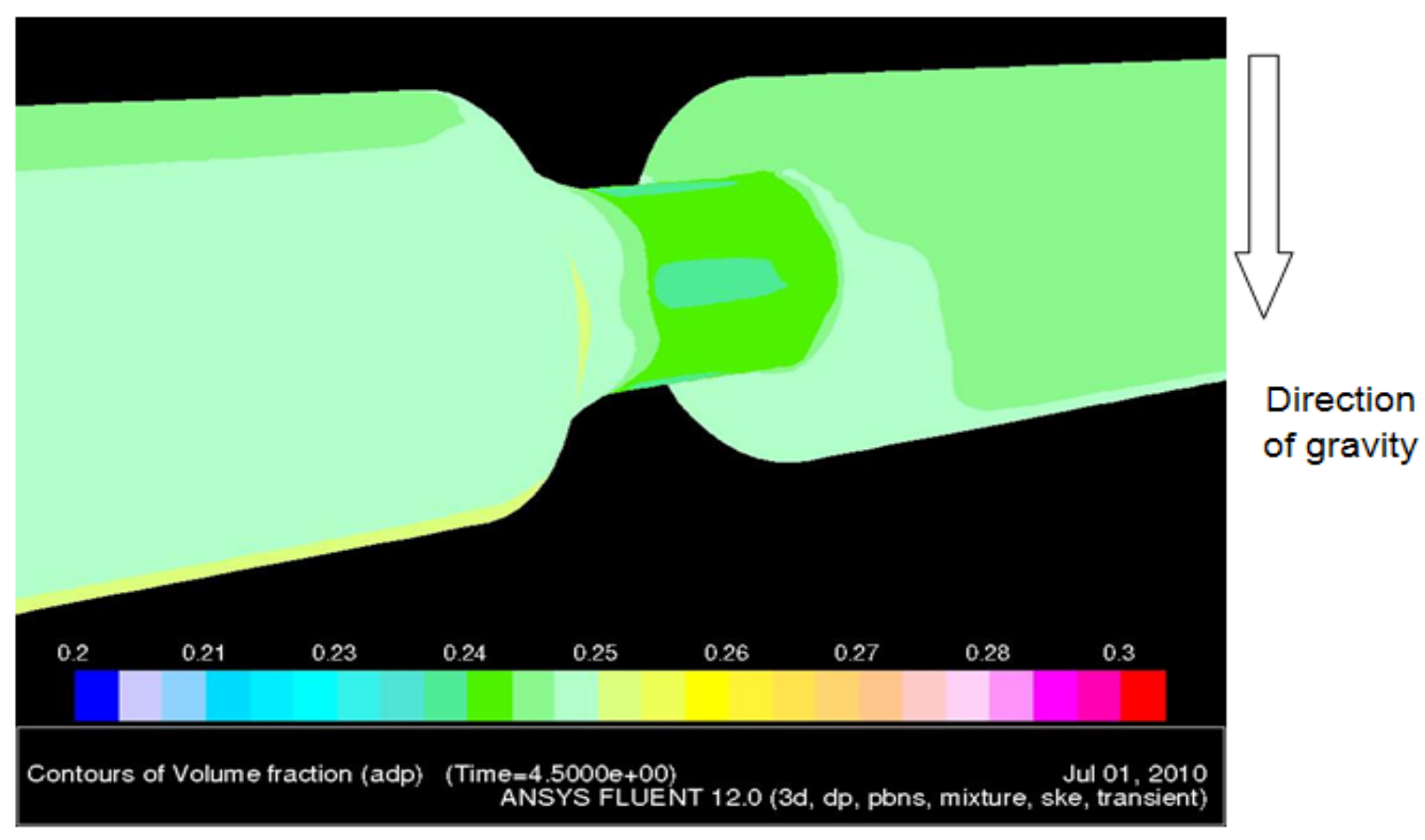

Figure 4.4.1.1: Contours of volume fraction for a slurry (ADP-xylene) containing $38 \mu \mathrm{m}$ ADP particles at an initial efflux concentration of 0.3 at $R_{D}=140000$ 


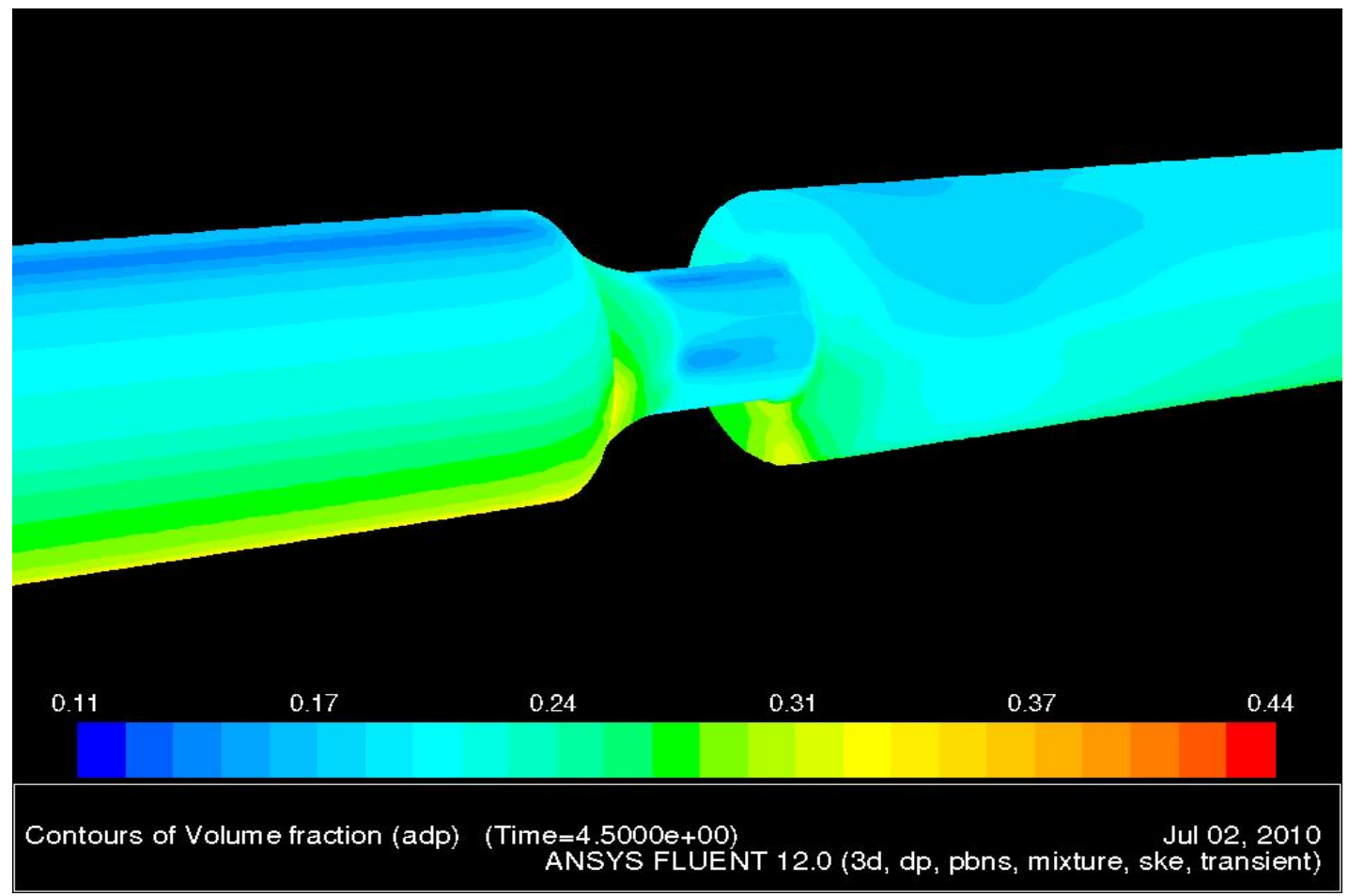

Figure 4.4.1.2: Contours of volume fraction for a slurry (ADP-xylene) containing $75 \mu \mathrm{m}$ ADP particles at an initial efflux concentration of 0.3 at $\operatorname{Re}_{D}=140000$

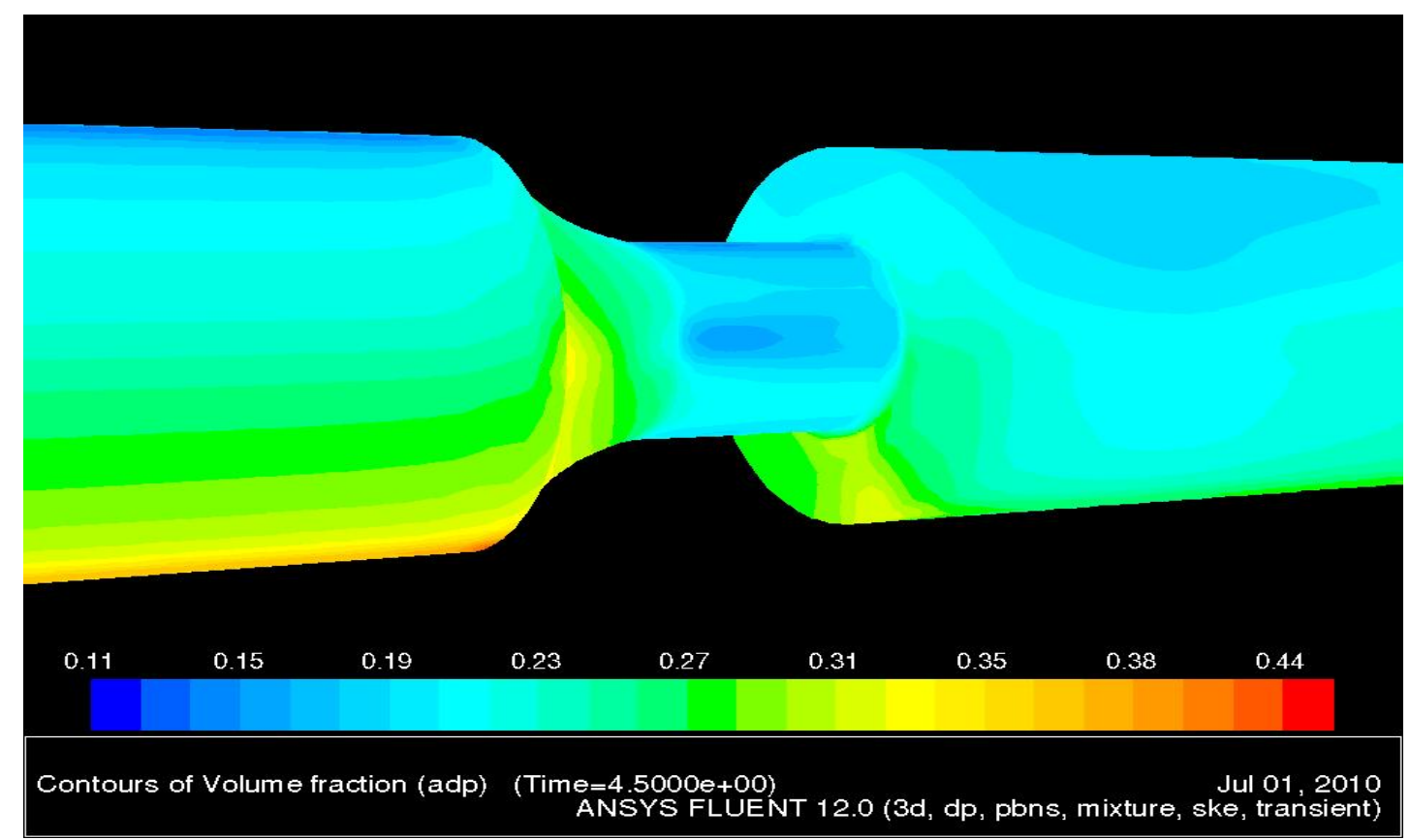

Figure 4.4.1.3: Contours of volume fraction for a slurry (ADP-xylene) containing $150 \mu \mathrm{m}$ ADP particles at an initial efflux concentration of 0.3 at $R_{D}=140000$ 
One can clearly observe the color gradient along the vertical diameter of the pipe increases with particle diameter. One can also observe that there is a higher degree of asymmetry in the fully developed region when compared to the throat section of the nozzle. The asymmetry may be attributed in part to the convergence criteria selected in FLUENT. The default convergence criteria and the under relaxation factors are too strict, and will result in the convergence of the solution only when the initial guesses are very accurate. Since an implicit solver was employed, it is not imperative for one to provide accurate initial guesses, nor is it essential to use the default under relaxation factors. Thus, one is free to adjust these factors so that the solution converges steadily. This is common practice, and is even recommended by the FLUENT manual. The asymmetry may also be attributed to the unstructured nature of the grid that is prone to the generation of distorted cells. Cross section contours for $150 \mu \mathrm{m}$ ADP particles are presented in Fig 4.4.1.4 and Fig 4.4.1.5.

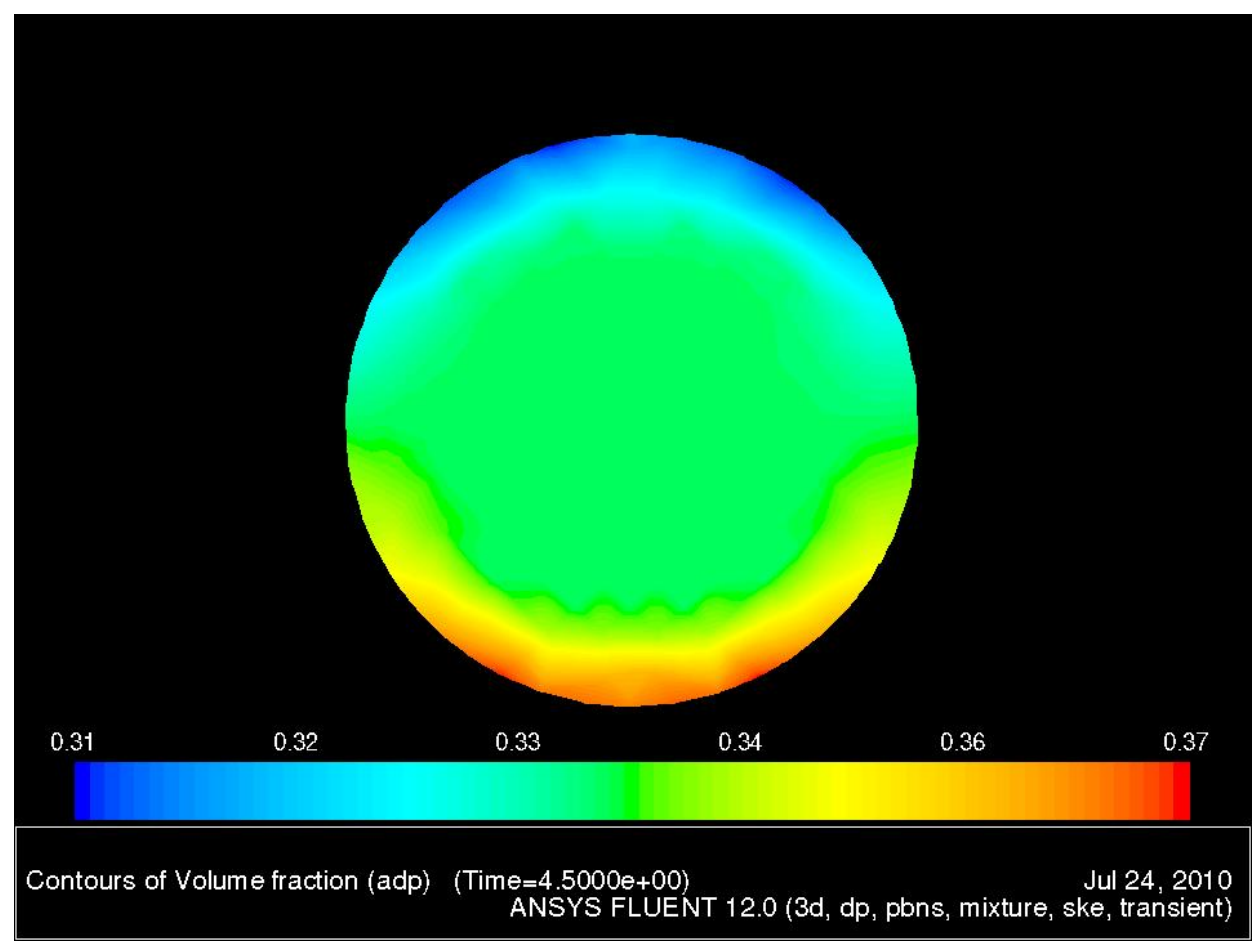

Figure 4.4.1.4: Cross section contours of volume fraction at the fully developed region for a slurry (ADP-xylene) containing $150 \mu \mathrm{m}$ ADP particles at an initial efflux concentration of 0.3 at $\operatorname{Re}_{\mathrm{D}}=140000$ 


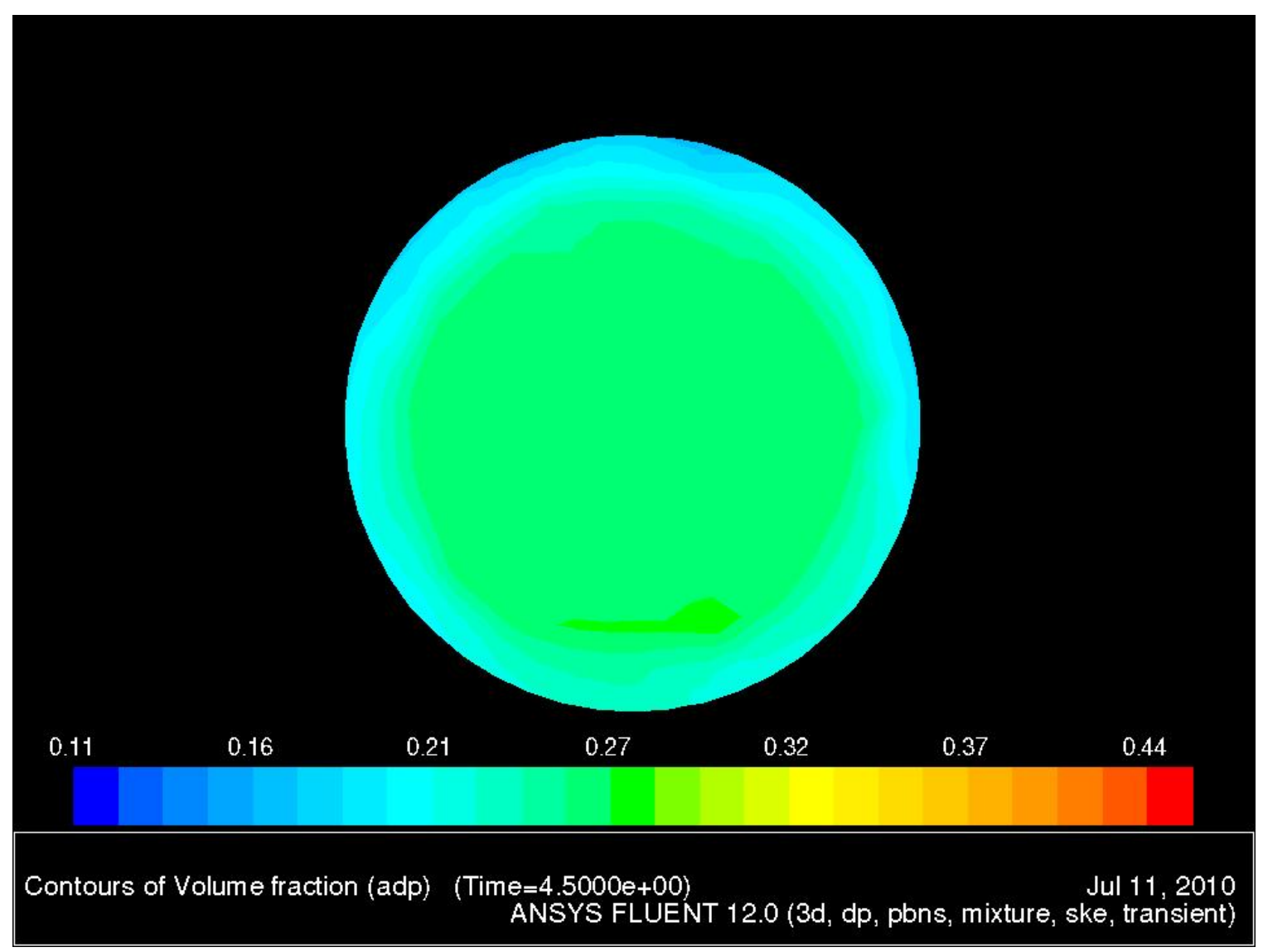

Figure 4.4.1.5: Cross section contours of volume fraction at the exit plane for a slurry (ADPxylene) containing $150 \mu \mathrm{m}$ ADP particles at an initial efflux concentration of 0.3 at $\operatorname{Re}_{\mathrm{D}}=\mathbf{1 4 0 0 0 0}$

\subsubsection{Contours of volume fraction $\left(\operatorname{Re}_{\mathrm{D}}=280000\right)$}

Similar to the previous section, the contours will be presented in the increasing order of the particle size and initial efflux concentration. Cross section volume fraction contours for a slurry containing $150 \mu \mathrm{m}$ ADP particles are also presented in this section. The gravity acts in the negative $\mathrm{Y}$ direction for all the figures presented in this section. 


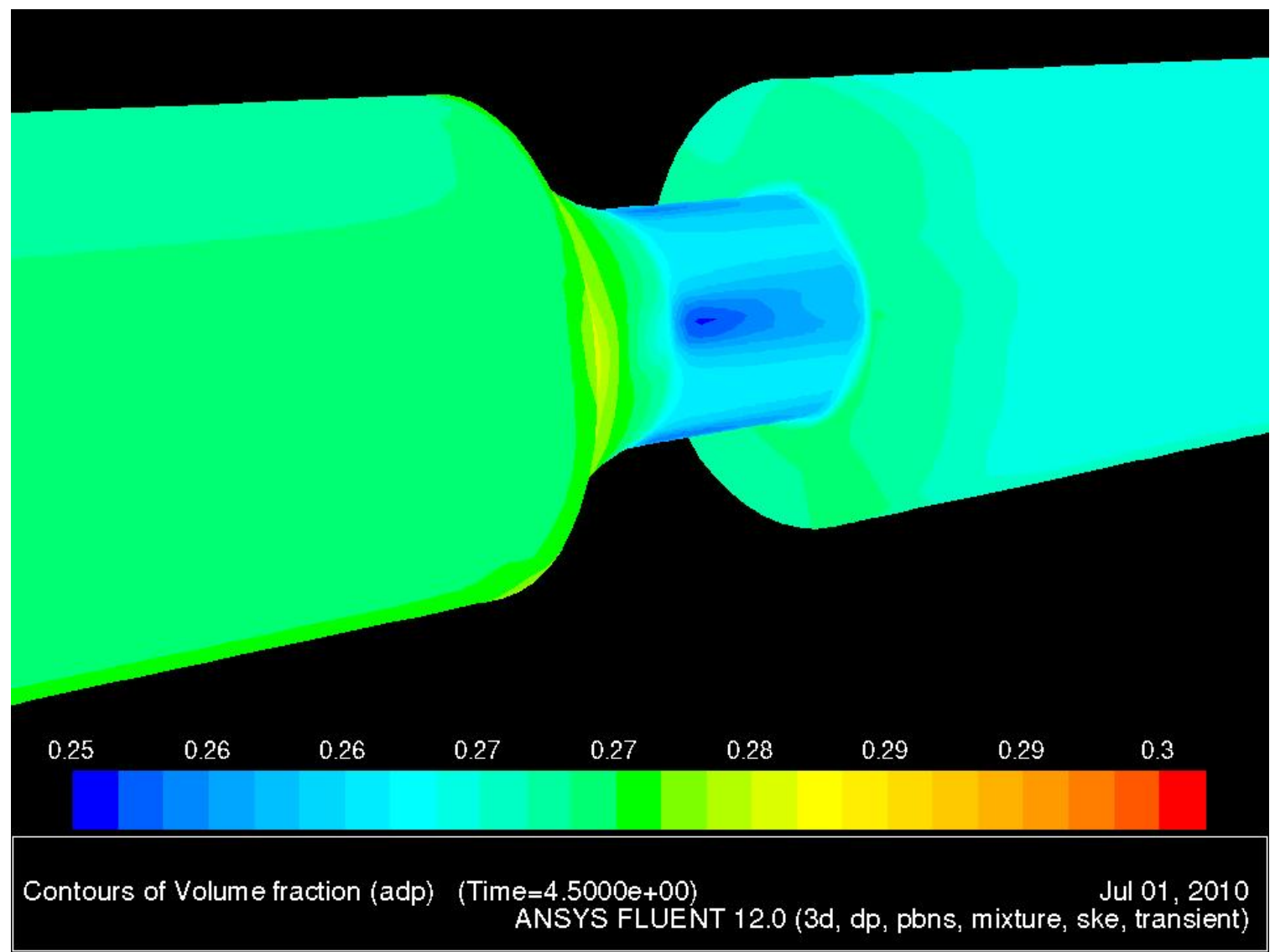

Figure 4.4.2.1: Contours of volume fraction for a slurry (ADP-xylene) containing $38 \mu \mathrm{m}$ ADP particles at an initial efflux concentration of 0.3 at $\mathbf{R e}_{\mathrm{D}}=\mathbf{2 8 0 0 0 0}$ 


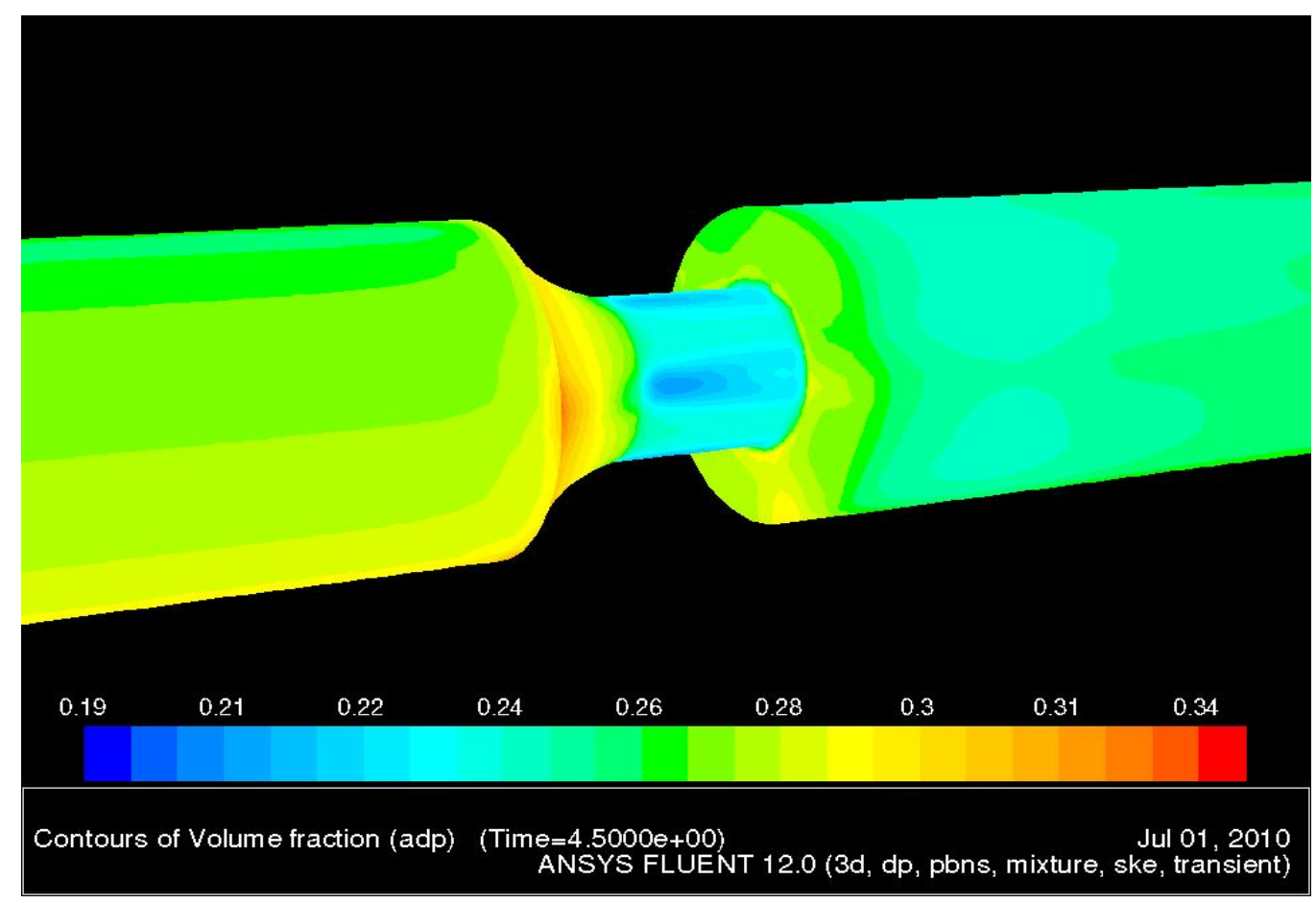

Figure 4.4.2.2: Contours of volume fraction for a slurry (ADP-xylene) containing $75 \mu \mathrm{m}$ ADP particles at an initial efflux concentration of 0.3 at $\mathbf{R e}_{\mathrm{D}}=\mathbf{2 8 0 0 0 0}$

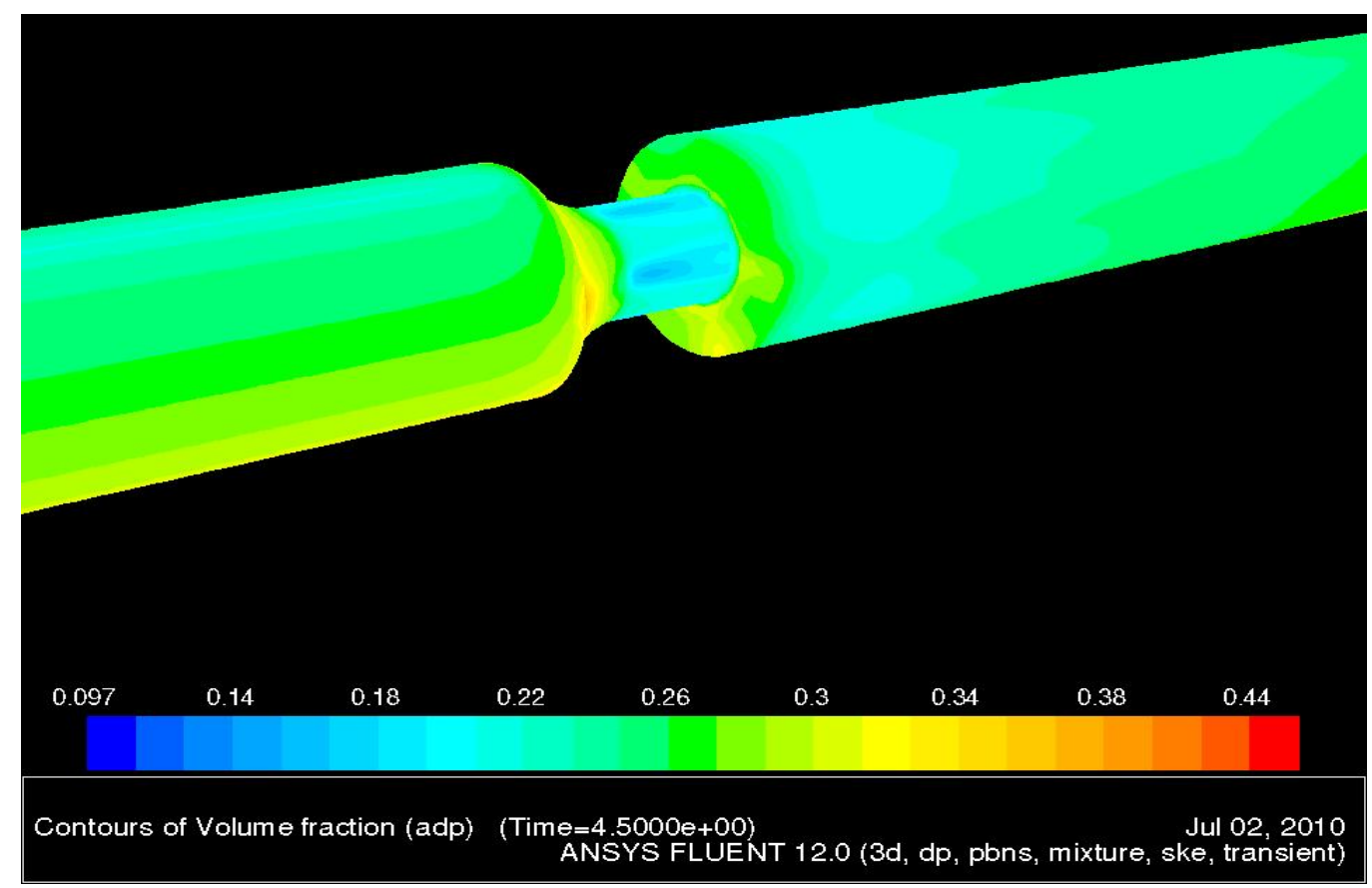

Figure 4.4.2.3: Contours of volume fraction for a slurry (ADP-xylene) containing $150 \mu \mathrm{m}$ ADP particles at an initial efflux concentration of 0.3 at $\mathbf{R e}_{\mathrm{D}}=\mathbf{2 8 0 0 0 0}$ 
Figures 4.4.2.4 and 4.4.2.5 present the cross-sectional volume fraction contours at the fully developed region and the exit plane.

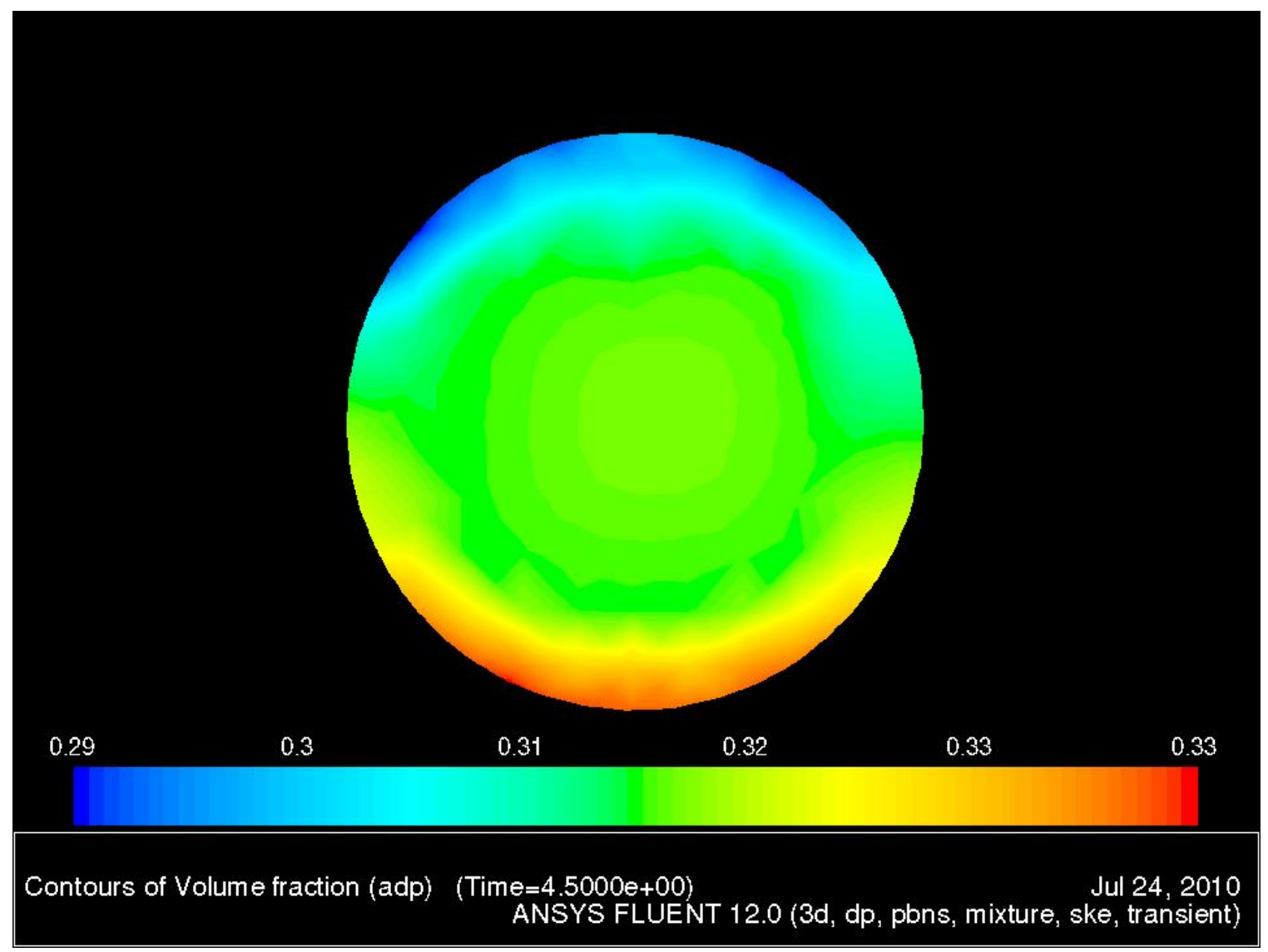

Figure 4.4.2.4: Cross section contours of volume fraction at the fully developed region for a slurry (ADP-xylene) containing $150 \mu \mathrm{m}$ ADP particles at an initial efflux concentration of 0.3 at $\operatorname{Re}_{\mathrm{D}}=280000$ 


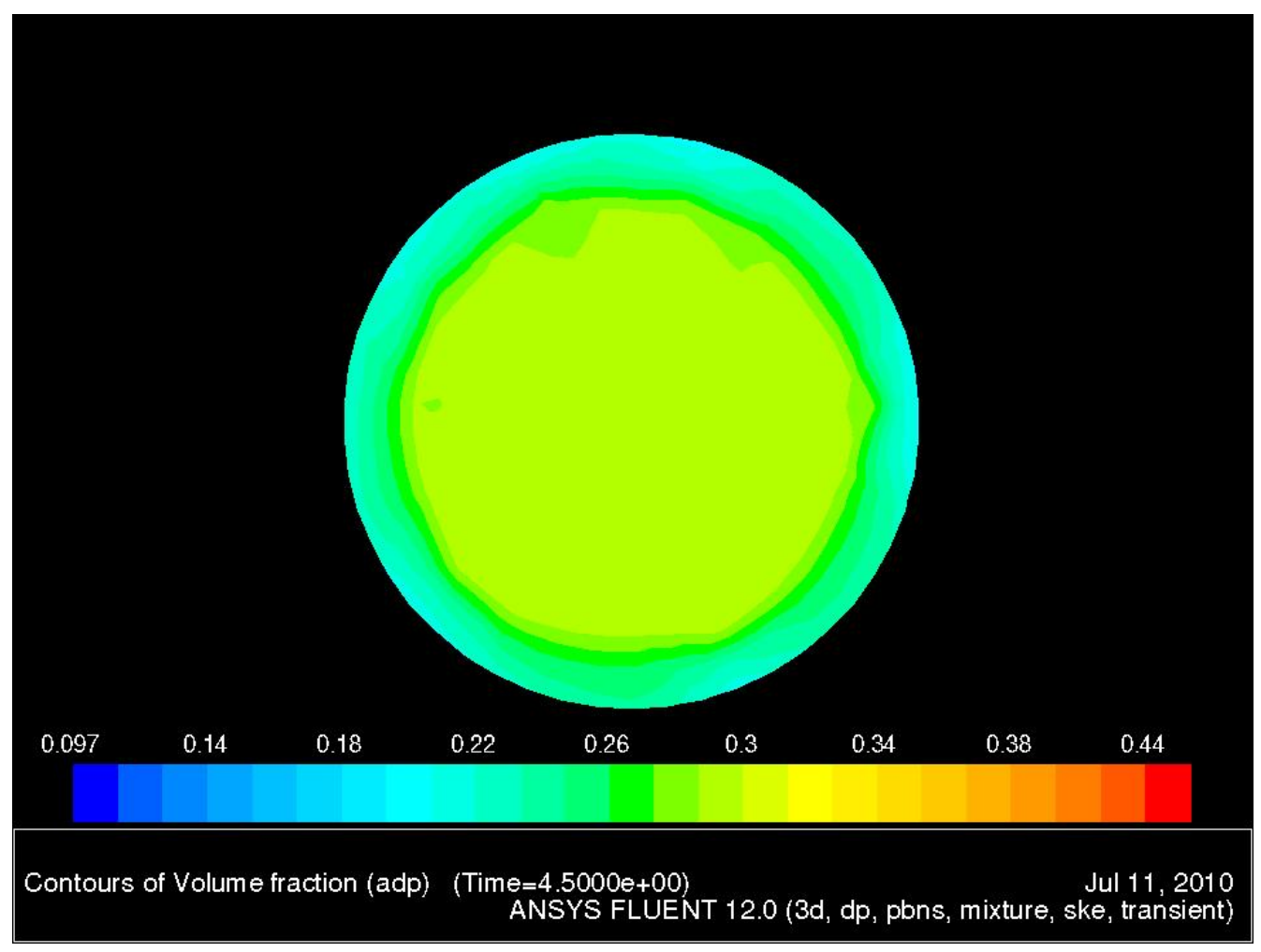

Figure 4.4.2.5: Cross section contours of volume fraction at the exit plane for a slurry (ADPxylene) containing $150 \mu \mathrm{m}$ ADP particles at an initial efflux concentration of 0.3 at

$$
\mathbf{R e}_{\mathrm{D}}=\mathbf{2 8 0 0 0 0}
$$

After observing the figures in this section one finds that the color gradient is smoother along the vertical diameter of the geometry, especially in case of the lighter particles (38 $\mu \mathrm{m}$ and 75 $\mu \mathrm{m})$. Further, if one devotes their attention to the throat section of the ASME flow nozzle, one would find that the volume fraction is almost constant throughout the cross section. This proves that, at least in case of the smaller particles, the concentration of the particles display reduced spatial variation as the slurry accelerates through the contraction. Again, the asymmetry might be due to the modification of the convergence criteria, under-relaxation factors in FLUENT, and the presence of distorted cells.

In conclusion, the concentration profiles of smaller particles display reduced spatial variation at the exit plane of the ASME flow nozzle. As the particle size increases, the concentration profiles tend to lose their uniformity owing to the effect of gravity. The shape of the concentration profiles registered at the exit plane of the flow nozzle is substantially unaltered by 
the increase in the initial efflux concentration. Further, as the Reynolds number $\left(\operatorname{Re}_{\mathrm{D}}\right)$ is increased, the concentration profiles of the smallest particles at the fully developed region and the exit plane display reduced spatial variation and are almost identical.

The exit plane of a symmetric contraction can serve as a suitable location for performing spectroscopic analysis of a slurry. Especially in case of a slurry where the average size of the suspended particles is less than $50 \mu \mathrm{m}$ because the results presented in this study indicate that contraction causes the slurry to exhibit characteristics that are representative of the total slurry volume. Further, the reduction in turbulence makes the region more conducive for performing near infra-red and mass spectroscopic analysis. 
CHAPTER V

\section{CONCLUSIONS AND RECOMMENDATIONS}




\subsection{Conclusions}

Near infra-red and mass spectroscopic analysis of a flowing slurry has rarely been documented, for it is a challenging task. Both the aforementioned spectroscopic techniques require the concentration profiles of a particle-laden slurry to be as uniform as practically possible. Uniform concentration profiles ensure that the slurry sample observed/withdrawn at the sampling location is representative of the total slurry volume. Since the contraction causes the velocity profiles of a fluid to display greater uniformity, its effect on the concentration profiles of a two phase flow was investigated through computational fluid dynamics. This gave rise to the following conclusions:

1. The present computational model was validated for slurry velocity profiles and secondary phase concentration profiles with the experimental data available in the literature.

2. In order to comprehend the effect of a contraction, a range of test cases (see Table 4.4.1) were simulated. The concentration profiles of the secondary phase were predicted and compared at the fully developed region of the geometry and the exit plane of the ASME flow nozzle.

3. It was found that the effect of the contraction was more pronounced in the case of heavier particles $\left(\mathrm{d}_{\mathrm{p}}=150 \mu \mathrm{m}\right)$. However, in all the test cases the concentration profiles of the secondary phase (ADP particles) displayed greater uniformity.

4. It was shown that the contraction causes enormous increase in the mean shear rate in the direction of the main flow. This is in good agreement with theory.

5. It was also illustrated that the contraction causes the turbulence intensity in the axial direction to drop. It is owing to these sorts of combined effects that the concentration profiles display greater uniformity. 
6. Since the concentration profiles display a certain degree of uniformity at the exit plane of the nozzle, this location can prove to be most suitable for performing near-infrared spectral or mass spectral analysis of a particle-laden slurry.

\subsection{Recommendations}

1. An obvious recommendation would be to use direct numerical simulation (DNS) or large eddy simulation (LES) or detached eddy simulation (DES) to increase the accuracy of the obtained solutions. One can also use Reynolds stress models to move beyond the Boussinesq eddy viscosity hypothesis.

2. The k-epsilon turbulence model with standard wall functions was used for the simulations; the model performs satisfactorily well to fathom the effect of gravity and the wall. Thus it is recommended that the near wall region should be modeled such that the condition $\mathrm{y}^{+} \sim 1$ is satisfied. This would enable one to employ the k-omega turbulence model which is considered more efficient in the near-wall region.

3. When the concentration profiles generated by employing a fine and a coarse mesh, both of which satisfied the constraints of the turbulence model employed, were compared, the results varied by a small margin, maximum error of $2 \%$. However, the velocity profiles for the coarse and the fine grid were in excellent agreement with each other. This anomaly was attributed to the value of $\mathrm{y}^{+}$or the presence of distorted cells. It seems that the concentration profiles generated by incorporating the mixture model are sensitive to the variations in the value of $\mathrm{y}^{+}$or the presence of distorted cells. It is recommended that the model constants should be adjusted to obtain more accurate results. It is also recommended that one use the Euler multi-phase model to predict the concentration profiles. The Euler multi-phase model is known to produce better results, but is memory intensive. 


\section{REFERENCES}

Batchelor, G. K., and Proudman, I. (1954) The effect of rapid distortion of a fluid in turbulent motion, Q J Mechanics Appl Math, 7, 83-103.

Blanco, M., Coello, J., Iturriaga, H., Maspoch, S., and Pazuela, C. (1998) Near-infrared spectroscopy in pharmaceutical industry, The Analyst, 123, 135-150.

Blanco, M., and Villarroya, I. (2002) NIR spectroscopy: a rapid-response analytical tool, TrAC Trends in Analytical Chemistry, 21, 240-250.

Boggess, B. (2001) Mass Spectrometry Desk Reference (Sparkman, O. David), Journal of Chemical Education, 78, 106-168.

Bradshaw, P. (1994) Turbulence: an outstanding difficulty of our subject, Experiments in Fluids, 16, 203-216.

Chandel, S., Seshadri, V., and Singh, S. N. (2009) Effect of additive on pressure drop and rheological characteristics of fly ash slurry at high concentration, Particulate Science and Technology: An International Journal, 27, 271 - 284.

Chang, C., and Powell, R. L. (2002) Hydrodynamic transport properties of concentrated suspensions, AIChE Journal, 48, 2475-2480.

Chen, I. Y., Chu, M.-C., Liaw, J.-S., and Wang, C.-C. (2008) Two-phase flow characteristics across sudden contraction in small rectangular channels, Experimental Thermal and Fluid Science, 32, 1609-1619.

Clark, J. (2000) private communication, Chemguide, United Kingdom.

Cho, D. S. (2010) private communication, University of Tennessee, Knoxville, TN.

Cody, R. B., Laramée, J. A., and Durst, H. D. (2005) Versatile new ion source for the analysis of materials in open air under ambient conditions, Analytical Chemistry, 77, 22972302 .

Crowe, C. T., Sommerfeld, M., and Tsuji, Y. (1998) Multiphase flows with droplets and particles, CRC Press LLC, Boca Raton, FL.

Ekambara, K., Sanders, R. S., Nandakumar, K., and Masliyah, J. H. (2009) Hydrodynamic simulation of horizontal slurry pipeline flow using ANSYS-CFX, Industrial \& Engineering Chemistry Research, 48, 8159-8171. 
Emmons, H. W. (1970) Critique of numerical modeling of fluid-mechanics phenomenon, Annual Review of Fluid Mechanics, 2, 15-36.

Fenn, J., Mann, M., Meng, C., Wong, S., and Whitehouse, C. (1989) Electrospray ionization for mass spectrometry of large biomolecules, Science, 246, 64-71.

Friesen, W. I. (1996) Qualitative analysis of oil sand slurries using on-line NIR spectroscopy, Appied Spectroscopy, 50, 1535-1540.

Gillies, R. G., and Shook, C. A. (2000) Modeling high concentration settling slurry flows, The Canadian Journal of Chemical Engineering, 78, 709-716.

Gillies, R. G., Shook, C. A., and Xu, J. (2004) Modeling heterogeneous slurry flows at high velocities, The Canadian Journal of Chemical Engineering, 82, 1060-1065.

Hageman, J. A., Westerhuis, J. A., and Smilde, A. K. (2005) Temperature robust multivariate calibration: an overview of methods for dealing with temperature influences on near infra red spectra, Journal of Near Infrared Spectroscopy, 13, 53-62.

Hanks, R. W., and Dadia, B. H. (1971) Theoretical analysis of the turbulent flow of nonNewtonian slurries in pipes, AIChE Journal, 17, 554-557.

Hirsch, C. (2007) Numerical computation of internal and external flows, 2 ed., John Wiley \& Sons, Burlington, MA.

Kaushal, D. R., and Tomita, Y. (2003) A comparative study of pressure drop in multisized particulate slurry flow through pipe and rectangular duct, International Journal of Multiphase Flow, 29, 1473-1487.

Kaushal, D. R., and Tomita, Y. (2007) Experimental investigation for near-wall lift of coarser particles in slurry pipeline using $\gamma$-ray densitometer, Powder Technology, 172, 177-187.

Kline, S. J., Reynolds, W. C., Schraub, F. A., and Runstadler, P. W. (1967) The structure of turbulent boundary layers, Journal of Fluid Mechanics, 30, 741-773.

Libby, P. A. (1996) Introduction to Turbulence, Taylor \& Francis, Washington, D.C.

Liu, M., Chen, L., and Duan, Y. (2009) Local resistance characteristics of highly concentrated coal-water slurry flow through fittings, Korean Journal of Chemical Engineering, 26, 569-575.

Moffatt, H. K. (2002) G.K. Batchelor and the homogenization of turbulence, Annual review of fluid mechanics, 34, 19-35.

Poloski, A., Adkins, H., Abrefah, J., Casella, A., Hohimer, R., Nigl, F., Minette, M., Toth, J., Tingey, J., and Yokuda, S. (2009) Deposition velocities of Newtonian and non-Newtonian slurries in pipelines, U.S. Department of Energy (DOE) DE-AC05-76RL01830, Pacific Northwest National Laboratory, Richland, WA.

Pope, S. B. (2000) Turbulent Flows, Cambridge University Press, New York, NY. 
Robb, D. B., Covey, T. R., and Bruins, A. P. (2000) Atmospheric pressure photoionization: An ionization method for liquid chromatography: mass spectrometry, Analytical Chemistry, 72, 3653-3659.

Roco, M. C., and Shook, C. A. (1984) Computational method for coal slurry pipelines with heterogeneous size distribution, Powder Technology, 39, 159-176.

Savill, A. M. (1987) Recent developments in rapid-distortion theory, Annual Review of Fluid Mechanics, 19, 531-573.

Scarff, M., Arnold, S. A., Harvey, L. M., and McNeil, B. (2006) Near infrared spectroscopy for bioprocess monitoring and control: current status and future trends, Critical Reviews in Biotechnology, 26, 17 - 39.

Schaan, J., Gillies, R. G., and Shook, C. A. (2000) The effect of particle shape on pipeline friction for Newtonian slurries of fine particles, Canadian Journal of Chemical Engineering, $78,717-725$.

Stickel, J. J., and Powell, R. L. (2005) Fluid mechanics and rheology of dense suspensions, Annual Review of Fluid Mechanics, 37, 29-49.

Switalski, S. C., Colin, T., Redden, N., Stahlecker, E., and Parthasarathy, V. (1999) Nearinfrared diffuse reflectance fiber optic spectroscopy for process monitoring applications, Proceedings of SPIE- The International Society of Optical Engineering, 3538, 239-258.

Tokaty, G. A. (1994) A history and philosophy of fluid mechanics, Dover publications, Mineola, NY.

Vaidyanathan, S., Macaloney, G., and McNeil, B. (1999) Fundamental investigations on near-infrared spectra of microbial biomass as applicable to bioprocess monitoring, The Analyst, 124, 154-167.

Wilcox, D. C. (1993) Turbulence modeling for CFD, 3 ed., DCW Industries, La Canada, CA.

Wildman, D. J., Ekmann, J. M., Kadambi, J. R., and Chen, R. C. (1992) Study of the flow properties of slurries using the refractive index matching technique and LDV, Powder Technology, 73, 211-218.

White, F. M. (2006) Viscous Fluid Flow, 3 ed., McGraw -Hill, New York, NY. 
APPPENDIX

EXPERIMENTAL SETUP 
This section lays the foundations for an experimental facility that will have the capability to accommodate spectral (near-infrared and mass) of a multi-phase flow. A prerequisite for this experimental facility is that it should be capable of handling a multi-phase flow. The following are the conditions that the facility must satisfy.

\begin{tabular}{|c|c|}
\hline Primary phase & Water \\
\hline Secondary phase & Glass beads \\
\hline Mean size of secondary phase $\left(d_{p}\right)$ & $50 \mu \mathrm{m}$ \\
\hline Maximum secondary phase concentration & $15 \%$ (volumetric) \\
\hline Average velocity of the primary phase & $2 \mathrm{~m} / \mathrm{s}$ \\
\hline Total volume of the working fluid & 150 gallons \\
\hline
\end{tabular}

Table A1: Operational parameters of the experimental setup

The heart of the aforementioned facility is the slurry pump that will pump the fluid through the pipelines. Thus a suitable pump capable of handling the particle laden slurry has to be sized.

AFT Fathom 7.0 was employed to develop a system curve for the hydraulic pipe system handling the slurry. A range of dealers were contacted in order to find a suitable bump that would meet both the design and budget constraints. Pump curves were extracted from the designer catalogues, and were plotted along with the system curves. For certain pumps the point of intersection of the system and the pump curves fell in the region where the efficiency of the pump would be hampered in the long run. For one particular pump this point fell in the safe zone. This pump was selected and procured. The pump curve and the system curve are presented in Fig A1. 


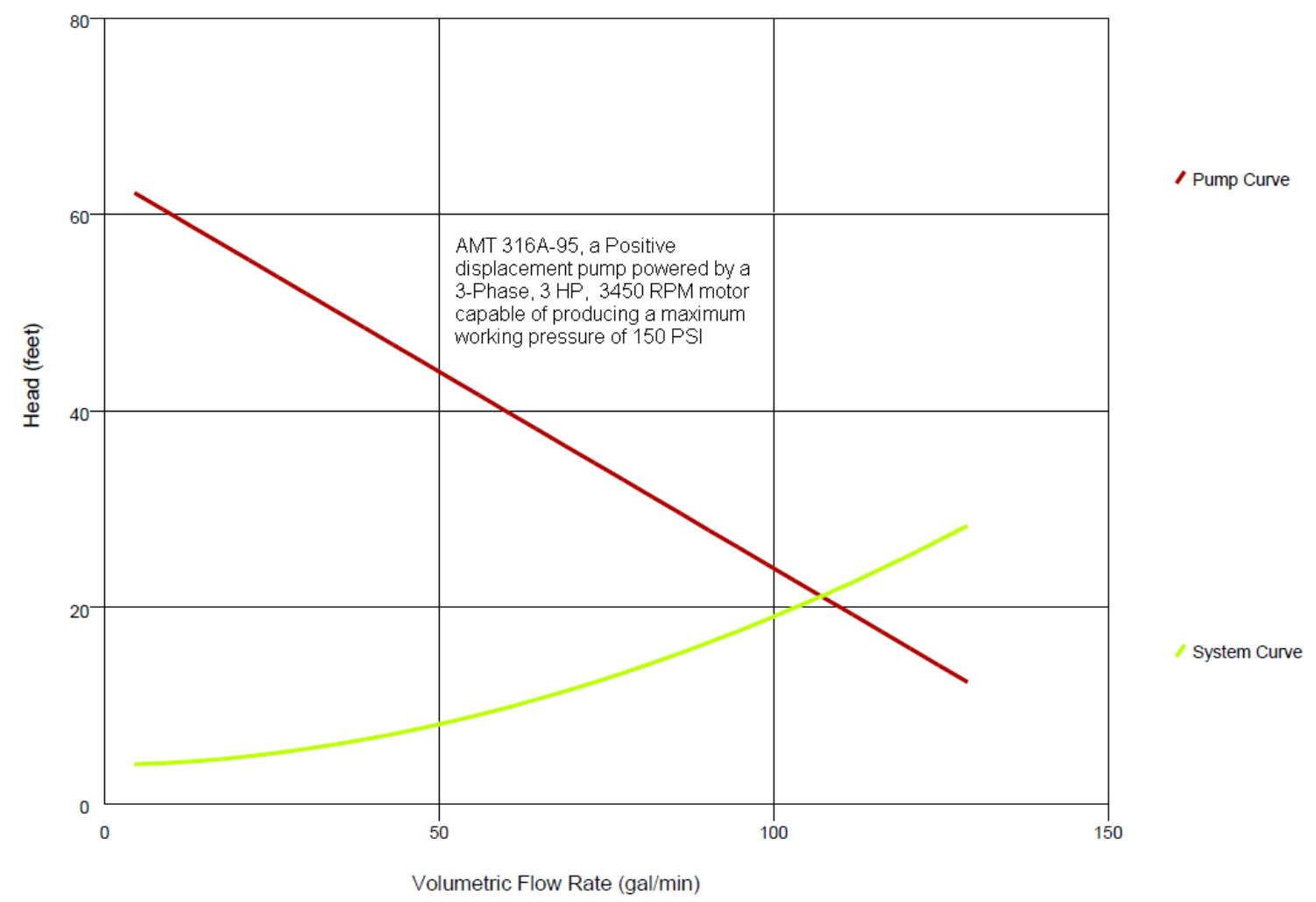

Figure A1: Pump curve Vs. system curve

The plumbing equipment was purchased from a local dealer. Two clear plastic cast acrylic tubes that would serve the purpose of a test section were purchased. Since the spectral measurements are sensitive to vibrations, a strut frame was constructed. This frame served the two purposes. First, it provided a firm support to the plumbing and also to the test section. Second, they raised the recirculation loop to a convenient elevation. This made the experimental setup conducive to spectral analysis. An isometric view of the experimental setup is presented in Fig A2. 


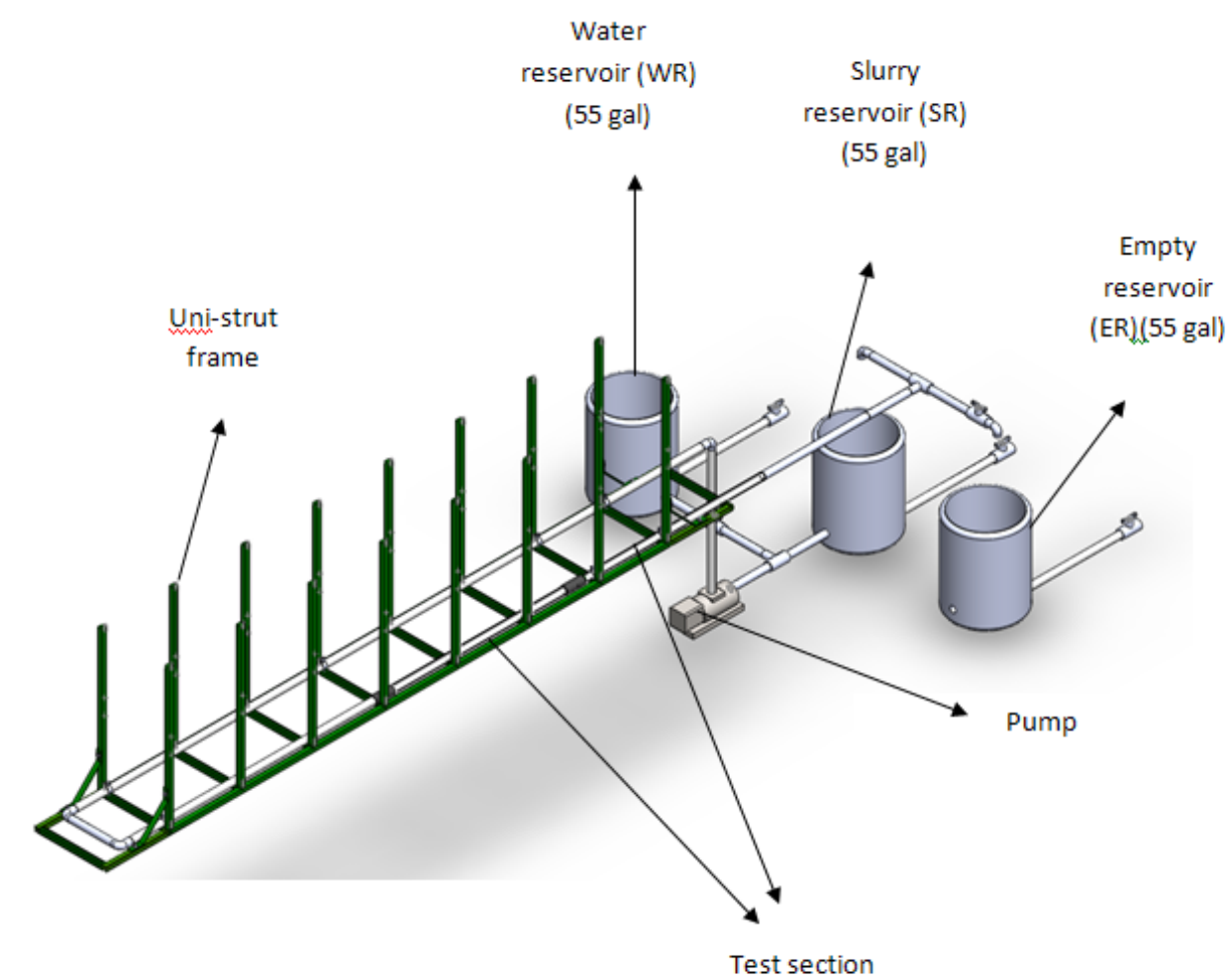

4

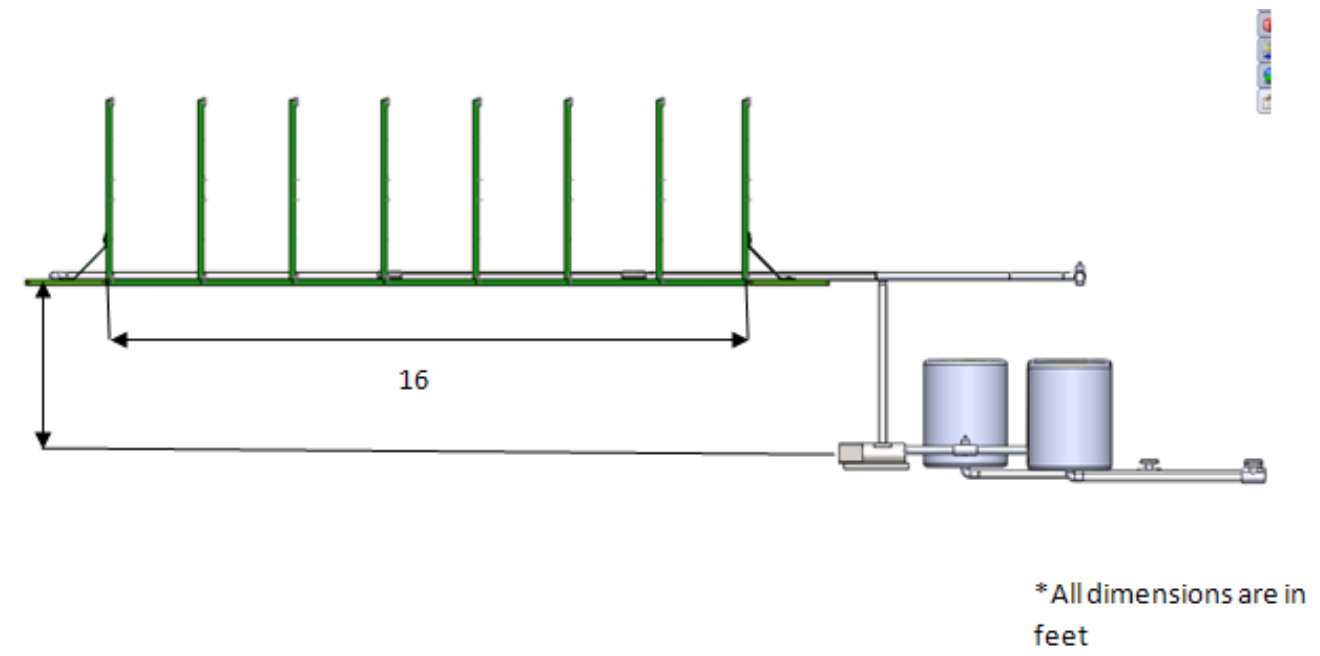

Figure A2: Proposed experimental facility 
VITA

Abhishek Deshpande

Candidate for the Degree of

Master of Science

Thesis: EFFECT OF SYMMETRIC CONTRACTION ON THE CONCENTRATION ON THE CONCENTRATION PROFILES OF A PARTICLE-LADEN SLURRY

Major Field: Mechanical Engineering

Biographical:

Education:

Completed the requirements for the Master of Science in Mechanical Engineering at Oklahoma State University, Stillwater, Oklahoma in December, 2010.

Completed the requirements for the Bachelor of Technology in your Mechanical Engineering at Jawaharlal Nehru Technological University, Hyderabad, Andhra Pradesh, India June 2008.

Experience:

Design Intern: December 2007-May 2008

Research Center Imarat, Hyderabad, Andhra Pradesh, India

Teaching Assistant: August 2008-May 2010

Department of Aerospace and Mechanical Engineering

Oklahoma State University

Research Assistant:August 2009-August 2010

Department of Aerospace and Mechanical Engineering

Oklahoma State University

Professional Memberships:

Member of American Society of Mechanical Engineers (ASME)

Member of American Society of Heating, Refrigerating and Air-Conditioning Engineers (ASHRAE) 

CONCENTRATION PROFILES OF A PARTICLE-LADEN SLURRY

Scope and Method of Study: The effect of a symmetric contraction on the concentration profiles of a xylene- 2 amino, 4, 6 dimethyl pyrimidine (ADP) slurry flowing in a cylindrical pipe was simulated using computational fluid dynamics. ANSYS FLUENT 12.0 was used as the computational fluid dynamic software. A variation in the pipe Reynolds number, initial efflux concentration, and the particle size were considered. An ASME flow nozzle, that served the purpose of a contraction, was incorporated in the fully-developed region of the pipe geometry. The pipe had an L/D ratio of 55. The k-epsilon turbulence model with standard wall functions coupled with the mixture multi-phase model was used to simulate all the test cases. A grid independence study was performed for both the velocity and concentration profiles. The model was validated with experimental data sets available in the literature. The concentration profiles at the exit plane were compared with those registered at the fully-developed region.

Findings and Conclusions: The present computational model predicts the velocity and the concentration profiles of a slurry with an acceptable degree of accuracy. The accuracy of the simulated results is compromised in the near-wall region. It was found that the concentration profiles in all the test cases displayed reduced spatial variation at the exit plane of the geometric contraction. At higher Reynolds number $\left(\mathrm{Re}_{\mathrm{D}}\right)$ the concentration profiles of $38 \mu \mathrm{m}$ ADP particles realized at the exit plane and the fully-developed region were almost identical. It was found that the gradient of concentration along the vertical diameter for heavier particles decreases with an increase in pipe Reynolds number. The turbulence intensity of the flow field at the exit plane of the contraction was found to be lower than that registered at the fully-developed region. Owing to this, the exit plane of the geometric contraction may serve as a suitable location for performing near infrared and mass spectral analysis of a particle-laden slurry. 